\title{
Behavioral Responses of Invertebrate Larvae to Water Column Cues
}

\author{
by \\ Jeanette Danielle Wheeler \\ Submitted to the Joint Program in Biological Oceanography \\ in partial fulfillment of the requirements for the degree of \\ Doctor of Philosophy \\ at the \\ MASSACHUSETTS INSTITUTE OF TECHNOLOGY \\ and the \\ WOODS HOLE OCEANOGRAPHIC INSTITUTION
}

February 2016

(C)2016 Jeanette D. Wheeler.

All rights reserved.

The author hereby grants to MIT and WHOI permission to reproduce and to distribute publicly paper and electronic copies of this thesis document in whole or in part in any medium now known or hereafter created.

Author

Joint Program in Biological Oceanography Massachusetts Institute of Technology \& Woods Hole Oceanographic Institution January 8, 2016

Certified by

Lauren Mullineaux Senior Scientist, Department of Biology Woods Hole Oceanographic Institution

Thesis Supervisor

Certified by

Karl Helfrich

Senior Scientist, Department of Physical Oceanography Woods Hole Oceanographic Institution

Thesis Supervisor

Accepted by

Lauren Mullineaux

Chair, Joint Committee for Biological Oceanography Massachusetts Institute of Technology Woods Hole Oceanographic Institution 


\title{
Behavioral Responses of Invertebrate Larvae to Water Column Cues
}

\author{
by \\ Jeanette Danielle Wheeler
Submitted to the Joint Program in Biological Oceanography
Massachusetts Institute of Technology
\& Woods Hole Oceanographic Institution
on January 8, 2016, in partial fulfillment of the
requirements for the degree of
Doctor of Philosophy

\begin{abstract}
Many benthic marine invertebrates have two-phase life histories, relying on planktonic larval stages for dispersal and exchange of individuals between adult populations. Historically, larvae were considered passive drifters in prevailing ocean currents. More recently, however, the paradigm has shifted toward active larval behavior mediating transport in the water column. Larvae in the plankton encounter a variety of physical, chemical, and biological cues, and their behavioral responses to these cues may directly impact transport, survival, settlement, and successful recruitment.

In this thesis, I investigated the effects of turbulence, light, and conspecific adult exudates on larval swimming behavior. I focused on two invertebrate species of distinct morphologies: the purple urchin Arbacia punctulata, which was studied in pre-settlement planktonic stages, and the Eastern oyster Crassostrea virginica, which was studied in the competent-to-settle larval stage. From this work, I developed a conceptual framework within which larval behavior is understood as being driven simultaneously by external environmental cues and by larval age.

As no a priori theory for larval behavior is derivable from first principles, it is only through experimental work that we are able to access behaviors and tie them back to specific environmental triggers. In this work, I studied the behavioral responses of larvae at the individual level, but those dynamics are likely playing out at larger scales in the ocean, impacting population connectivity, community structure, and resilience. In this way, my work represents progress in understanding how the ocean environment and larval behavior couple to influence marine ecological processes.
\end{abstract}

Thesis Supervisor: Lauren Mullineaux

Title: Senior Scientist, Department of Biology

Woods Hole Oceanographic Institution

Thesis Supervisor: Karl Helfrich

Title: Senior Scientist, Department of Physical Oceanography

Woods Hole Oceanographic Institution 


\section{Acknowledgments}

This work was funded by the National Science Foundation under grant OCE-0850419 (to LS Mullineaux and KR Helfrich), by the National Oceanic and Atmospheric Administration Sea Grant NA14OAR4170074 (to LS Mullineaux, KR Helfrich, and JD Wheeler), grants from WHOI Coastal Ocean Institute, discretionary WHOI funds, a WHOI Ocean Life Fellowship (LS Mullineaux), and a Grove City College Swezey Fund Grant (EJ Anderson).

Thanks to current and past lab members Skylar Bayer, Meredith White, Stace Beaulieu, and especially Susan Mills for experimental assistance, mentorship, and support. Thanks to Roscoe, Chester, and Louie for unwavering devotion. Thanks to former summer students Amber Emerson, Elaine Luo, Brenna McGann, Andre Price, and Anthony Ritchie for their enthusiasm and hard work. Thanks go to Vicke Starczak, Jesús Pineda, and Houshuo Jiang for valuable assistance with data analysis and interpretation. Thanks to Rick Galat and the Facilities staff for ensuring the continued success of my seawater system, and to Dave Kulis for experimental assistance. Special thanks to Karen Chan for her constant interest, support, and collaborative spirit.

Thanks to my thesis committee: Erik Anderson, Mike Neubert, and Roman Stocker for your unwavering interest in my work and my continued success, and for your mentorship and guidance.

Thanks to my advisors, Lauren Mullineaux and Karl Helfrich, for giving me the opportunity to work with you for the last five years. You've both taught me so much about being a scientist, and I am grateful that I can carry that knowledge forward.

Thanks to my family and especially Joe Fitzgerald, without whom none of this would have been possible. 


\section{Contents}

1 Introduction $\quad 11$

1.1 The planktonic dispersal of larval invertebrates . . . . . . . . . . . 11

1.2 Ontogenetic changes in larval swimming and orientation of pre-competent sea urchin Arbacia punctulata in turbulence . . . . . . . . . . . . . . . 14

1.3 Upward swimming of competent oyster larvae Crassostrea virginica persists in highly turbulence flow as detected by PIV flow subtraction . . . . . . . . . 14

1.4 Isolating the hydrodynamic triggers of the dive response in eastern oyster larvae 15

1.5 Light stimulates swimming behavior of larval eastern oysters (Crassostrea virginica) in turbulent flow . . . . . . . . . . . . . . . . 16

1.6 Larval oyster swimming responses to adult chemical exudates: evidence for the desperate larva hypothesis? . . . . . . . . . . . . . . . . 16

2 Ontogenetic changes in larval swimming and orientation of pre-competent $\begin{array}{lr}\text { sea urchin Arbacia punctulata in turbulence } & 17\end{array}$

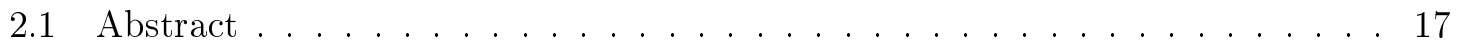

2.2 Introduction . . . . . . . . . . . . . . . . . . . 18

2.3 Materials and Methods . . . . . . . . . . . . . . . . . . . . 20

2.3.1 Study organism and larval culturing . . . . . . . . . . . . 20

2.3.2 Video observations of larval swimming in turbulence . . . . . . . . . 21

2.3.3 Larval tracking . . . . . . . . . . . . . . . . . 23

2.3.4 Flow subtraction and larval velocities computation . . . . . . . . . 24

2.3.5 Larval orientation . . . . . . . . . . . . . . . . 25

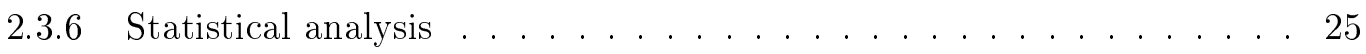

2.4 Results . . . . . . . . . . . . . . . . . . 26 
2.4.1 Larval size and swimming speed increased with ontogeny . . . . . . . 26

2.4.2 Swimming speeds changed with turbulence level but differed between

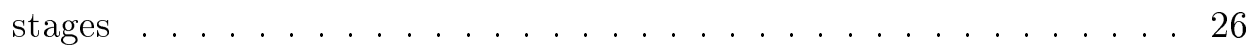

2.4.3 Stability decreased with turbulence and age . . . . . . . . . . 27

2.4.4 Swimming speed correlated with orientation angle . . . . . . . 28

2.5 Discussion . . . . . . . . . . . . . . . . . . 31

2.6 Acknowledgements . . . . . . . . . . . . . . . . . 35

2.7 Supplemental Material . . . . . . . . . . . . . . . . . . . 36

3 Upward swimming of competent oyster larvae (Crassostrea virginica) persists in highly turbulent flow as detected by PIV flow subtraction $\quad 37$

3.1 Abstract . . . . . . . . . . . . . . . . . 37

3.2 Introduction . . . . . . . . . . . . . . . . . . 38

3.3 Methodology . . . . . . . . . . . . . . . . . . . . 41

3.3.1 Experimental organism . . . . . . . . . . . . . . . . . 41

3.3 .2 Culturing of larvae . . . . . . . . . . . . . . . 41

3.3.3 Experimental tank . . . . . . . . . . . . . . . 41

3.3.4 Experimental Procedure . . . . . . . . . . . . . . . . . . 43

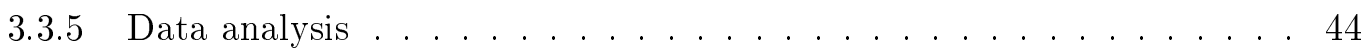

3.3.6 Statistical analysis . . . . . . . . . . . . . . . . 49

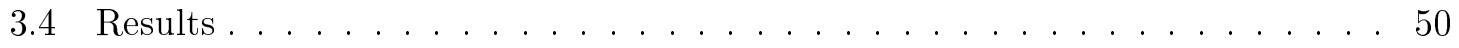

3.4.1 Energy dissipation rate . . . . . . . . . . . . . . . 50

3.4.2 Absolute versus isolated vertical swimming velocity . . . . . . . . . 52

3.4 .3 Vertical swimming velocity in turbulence . . . . . . . . . . 55

3.4 .4 Dive frequency in turbulence . . . . . . . . . . . . 56

3.5 Discussion . . . . . . . . . . . . . . . . 57

3.6 Acknowledgements . . . . . . . . . . . . . . . . . 61

4 Isolating the hydrodynamic triggers of the dive response in eastern oyster $\begin{array}{ll}\text { larvae } & 63\end{array}$

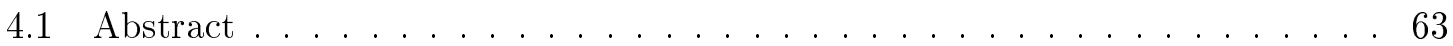

4.2 Introduction . . . . . . . . . . . . . . . . 64

4.3 Methods . . . . . . . . . . . . . . . . . 66 66 
4.3.1 Experimental organism and larval culturing . . . . . . . . . . . 66

4.3.2 Experimental set-up . . . . . . . . . . . . . . . 67

4.3.3 Larval tracking and local flow subtraction . . . . . . . . . . . 69

4.3.4 Identification of the dive response . . . . . . . . . . . . 70

4.3.5 Hydromechanical parameters detectable by larvae . . . . . . . . . . 71

4.3.6 Statistical analysis . . . . . . . . . . . . . . . . . . 74

4.4 Results . . . . . . . . . . . . . . . . . 76

4.4.1 Identification of dive response . . . . . . . . . . . . . . 76

4.4.2 Hydromechanical parameters triggering the dive response . . . . . . 77

4.5 Discussion . . . . . . . . . . . . . . . . . 81

4.6 Acknowledgements . . . . . . . . . . . . . . . . . . . 84

4.7 Supplemental Material . . . . . . . . . . . . . . . . . . . 86

4.7.1 Flow acceleration following larval trajectory . . . . . . . . . 86

4.7.2 Statistical analysis of non-acceleration turbulence fields as larval dive

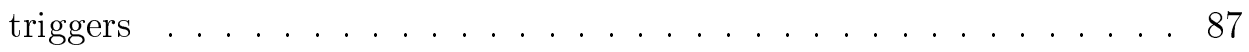

\section{Light stimulates swimming behavior of larval eastern oysters (Crassostrea} $\begin{array}{ll}\text { virginica) in turbulent flow } & 91\end{array}$

5.1 Abstract . . . . . . . . . . . . . . . . . . 91

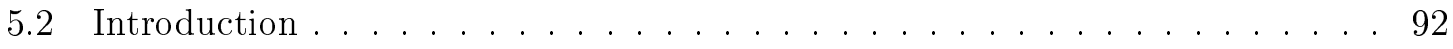

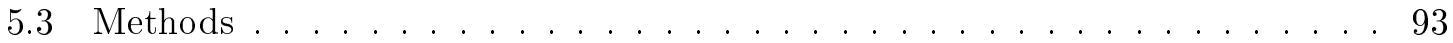

5.3 .1 Larval culture . . . . . . . . . . . . . . . . . . . . 93

$5.3 .2 \quad$ Experimental setup . . . . . . . . . . . . . . . . . 94

5.3.3 Local flow subtraction to isolate larval swimming velocities . . . . . 95

5.3 .4 Identification of dives . . . . . . . . . . . . . . . 96

5.3.5 Identification of helical swimming . . . . . . . . . . . . . 97

5.3 .6 Analysis of behavioral data . . . . . . . . . . . . . . . 97

5.4 Results . . . . . . . . . . . . . . . . . . . . 98

5.4 .1 Vertical swimming . . . . . . . . . . . . . . 98

5.4 .2 Probability of dives . . . . . . . . . . . . . . . . . . . . 99

5.4 .3 Helical swimming . . . . . . . . . . . . . . . . . . . . . 101

5.5 Discussion . . . . . . . . . . . . . . . . . . . 101 
5.6 Acknowledgements . . . . . . . . . . . . . . . . . . . . 104

5.7 Supplemental Material . . . . . . . . . . . . . . . . . . . . 105

6 Larval oyster swimming responses to adult chemical exudates: evidence $\begin{array}{ll}\text { for the desperate larva hypothesis? } & 107\end{array}$

6.1 Abstract . . . . . . . . . . . . . . . . . . . . 107

6.2 Introduction . . . . . . . . . . . . . . . . . . 108

6.3 Methods . . . . . . . . . . . . . . . . . . . . 110

6.3.1 Study species and handling practices . . . . . . . . . . . . . 110

6.3 .2 Experimental setup . . . . . . . . . . . . . . . 111

6.3 .3 Larval tracking . . . . . . . . . . . . . . . . . . . . 112

6.3.4 Statistical Analysis . . . . . . . . . . . . . . . . . . 113

6.4 Results . . . . . . . . . . . . . . . . . . . . . . . . 114

6.4 .1 Larval position . . . . . . . . . . . . . . . . 114

6.4 .2 Larval vertical velocity . . . . . . . . . . . . . . . . . 117

6.4.3 Larval growth and competency . . . . . . . . . . . . . . 120

6.5 Discussion . . . . . . . . . . . . . . . . . . . . . 124

6.6 Acknowledgements . . . . . . . . . . . . . . . . 127

7 Discussion $\quad 129$

7.1 Summary . . . . . . . . . . . . . . . . . . . . . 129

7.2 Synthesis . . . . . . . . . . . . . . . . . . . 131

7.2.1 External environmental cues affect larval behavior . . . . . . . . . . . 132

7.2.2 Larval aging affects larval behavior . . . . . . . . . . . . . . . . 134

7.2.3 Interactive effects of age and external cues on larval behavior . . . . . 134

7.2 .4 Extensions . . . . . . . . . . . . . . . . . . 135

7.2 .5 Broader Impacts . . . . . . . . . . . . . . . . . . . 137 


\section{Chapter 1}

\section{Introduction}

\subsection{The planktonic dispersal of larval invertebrates}

Many benthic marine invertebrates have two-phase life histories, relying on planktonic larval stages for dispersal and exchange of individuals between adult populations. The dispersal mode is prevalent in the marine world, as greater than $80 \%$ of described species (both vertebrate and invertebrate) spend a duration of time in the water column in an egg or larval form [1]. Historically, the large number of eggs/larvae produced by broadcast spawners was understood as a strategy to ensure population survival and growth: while large quantities of eggs were produced, only a random few would survive, due to the high larval mortality from predation and starvation. Longer pelagic durations meant larvae were exposed to these dangers for longer periods of time, and indeed species with the longest pelagic durations produce the most eggs [2]. However, this type of argument fails to account for the larval ability to increase survival chances through responses to water column cues [3]. The broad question of interest in this thesis is to understand how water column cues shape the landscape of ocean ecology. That is, how do physical and chemical processes elicit responses in larvae throughout the pelagic and settlement stages, and how do these responses mediate survival and success? Physical and chemical phenomena may be understood at least theoretically with governing equations of motion, while many biological phenomena, especially behavior, are only accessible experimentally. Relationships between environmental conditions and behavior may be investigated in the laboratory under a restricted range of conditions, but subsequently extrapolated using our more fundamental understanding of physical and chemical systems. Thus, in clarifying the relationships between environment and behavior, 
one may potentially understand and contextualize larval behaviors under a broad range of environmental conditions which are not accessible experimentally. To introduce this work, I begin by describing a general larval life history, the types of cues larvae may encounter during their larval duration, and the types of behavioral responses observed.

The majority of marine invertebrate species are broadcast spawners with long larval life stages [2], which constitutes the life history strategy which I focus on in the present study. Fewer $(<30 \%)$ species engage in brooding behaviors, retaining smaller clutches of eggs proximate to adult settlement sites. The planktonic period of a marine invertebrate encompasses spawning, hatching, and maturation through the various larval life stages. This time is spent passively drifting or free swimming in the plankton. Once larvae have matured to competency, or the final larval stage, they will settle out of the plankton and metamorphose at a benthic settlement site. "Settlement" is a term encompassing a considerable set of larval behaviors: it begins with the descent of a planktonic larva from the water column to the sea floor, after which the larva may either attach, move laterally to a suitable settlement habitat, or return up into the water column [4]. In other words, settlement describes all larval behavior occurring in the planktonic-benthic transition prior to the loss of swimming organs by metamorphosis. In this work, I focus separately on larval behaviors during 1) pre-competent planktonic life stages, and 2) the competent-to-settle final larval stage. I distinguish between these periods of the larval life history because larvae may experience one set of cues when aloft in the water column, and a different set when proximate to suitable settlement sites during competency.

Multiple invertebrate taxa display similar morphological characteristics and behavior during the planktonic period. Time spent in the plankton (the pelagic larval duration) ranges from minutes to months [5]. During this time, larvae mature through successive stages, with larval forms common among multiple taxa. Oyster larvae, for instance, mature in the plankton as free-swimming planktotrophs, passing through morphologically distinct trochophore, veliger, and pediveliger stages [6]. These specific larval forms are ubiquitous in invertebrate development: trochophores occur in molluscs, nemertea, sipunculids, and annelids, and veligers and pediveligers occur in other classes of molluscs [7]. More generally described larval characteristics, such as ciliated extensions (arms or lobes) for feeding and swimming are shared between diverse swimming planktotrophic invertebrate larvae, such as echinoids, molluscs, and polychaetes, and may represent a compromise for larvae that must 
simultaneously swim and feed [8]. Feeding mechanisms are also broadly shared by larvae of similar morphologies. Oyster larvae, for instance, feed via ciliary downstream collection, where ciliary beating on the extended velum sets up a current to draw particles towards the larval body, which are captured on the pre- and post-oral cirri and transferred to the mouth [9]. Ciliary downstream collection is also practiced by larvae of other bivalves, gastropods, and polychaetes [10].

The ubiquity of larval forms and behaviors is of interest when considering how behavior may play a role in larval dispersal during the planktonic period. Historically, larvae were considered passive drifters, helpless to counter the prevailing ocean currents and hence transported (with good fortune) to a suitable settlement site by purely hydrodynamic means (e.g., $[5,11])$. More recently, however, lab observations of larval motion in response to environmental factors were linked to field observations of distributions, leading to the new paradigm that larvae exert some active control over their own destinies via behavioral responses. In the water column, larvae encounter physical, chemical, and biological cues which may impact behavior (reviewed in $[12,3,13])$. A behavioral response of considerable importance is vertical swimming, which may allow larvae to regulate their water column position by overcoming vertical flow and/or their buoyancy differential from seawater. Self-governed vertical position in the water column affects large-scale dispersal patterns in simulations (e.g., [14, 15]) in coastal conditions where vertical flow velocities are not stronger than larval swimming capabilities [16]. During the planktonic phase, vertical position is relevant as its regulation can permit larvae to accumulate in, or escape from, vertically sheared flows or fronts. Such flows may facilitate larval transport to nearshore settlement environments $[17,18,19,20]$, or under different conditions, act as a barrier [21,22]. When larvae are preparing to settle, vertical position can be especially important in permitting them to identify and investigate suitable settlement sites (e.g., [23, 24, 4]). Some examples of physical cues for the regulation of vertical position include turbulence (Chapters 2, 3, 4, [25, 26, 27, 28, 29]), sound [30], and light (Chapter 5, [31, 32, 33, 34, 35]). Cues incorporating biological and chemical signals include exudates from predators [36, 37], prey or host species [38, 39], and conspecific settlement sites (Chapter 6, [40, 41]).

In this thesis, I examine how larvae at various life history stages respond to a range of physical and chemical cues that they might encounter during their planktonic period. The thesis is divided into five studies, summarized below, with an additional concluding 
chapter for discussion and final remarks. I begin with a study of larval urchin behavior during pre-competency and then transition into several studies of oyster larval behavior during competency. I will ultimately demonstrate that all these studies together offer insight into a conceptual model of general larval behavior, driven simultaneously by external environmental cues and larval aging.

\subsection{Ontogenetic changes in larval swimming and orientation of pre-competent sea urchin Arbacia punctulata in turbu- lence}

In Chapter 2, I investigate the role that turbulence plays in the motility of larval sea urchins (Arbacia punctulata) in two distinct larval stages. I observed swimming in 4-armed and older 6-armed pluteus larvae in a grid-stirred turbulence tank over a range of turbulence regimes. I used a recently developed particle image velocimetry technique which allowed me to simultaneously characterize both the motion of individual larvae and the fluid surrounding them, isolating the larval swimming velocity from advection in flow ${ }^{1}$. Initially, I expected the larger, heavier, six-armed larvae to be stronger swimmers and more stable to re-orientation by local shear. While six-armed larvae indeed swam faster than four-armed larvae, they were significantly less stable and were re-oriented frequently in turbulence. The changes to morphology-flow interactions with age suggest a mechanism for size and age selective transport of larvae.

\subsection{Upward swimming of competent oyster larvae Crassostrea virginica persists in highly turbulence flow as detected by PIV flow subtraction}

In Chapter 3, I shift focus from pre-competent to competent-to-settle larvae, and investigate a fundamental ecological question: how do competent larval oysters react to turbulent

\footnotetext{
${ }^{1}$ The organization of the thesis follows the succession of larval stages, hence Chapter 2 focuses on precompetent larvae while later chapters focus on competent-to-settle larvae. However, as Chapter 3 was the first published manuscript of the thesis, the technical details of the particle image velocimetry flow subtraction method are contained in that chapter, and the relevant reference is provided in Chapter 2. Readers are invited to read the Methods of Chapter 3 in conjunction with the Methods of Chapter 2, if desired.
} 
flow, and what does this imply about turbulence as a settlement cue for oyster larvae? I determined the effect of turbulence on vertical swimming velocity and diving responses in competent eastern oyster larvae (Crassostrea virginica), in a grid-stirred turbulence tank using a particle image velocimetry method similar to that described in Chapter 2. Contrary to my expectations of turbulence as a settlement cue, larvae swam upward even in highly turbulent flow, and the dive response became less frequent. These observations provide evidence that turbulence alone may not always be a sufficient cue for settlement out of the water column, and speaks to the physical capabilities of larvae in vigorous flow conditions. Further, my results suggest that reliable characterization of larval behavior in turbulent conditions requires the subtraction of local flow at an individual level, imposing the technical constraint of simultaneous flow and behavioral observations.

\subsection{Isolating the hydrodynamic triggers of the dive response in eastern oyster larvae}

In Chapter 4, I build on my previous understanding of larval oyster swimming in turbulence (Chapter 3), by identifying and isolating the hydrodynamic triggers to the dive response in competent oyster larvae. Diving may play an important role in active settlement, since diving larvae move rapidly downward in the water column and may regulate their proximity to suitable settlement sites. Using my experimental data of larval swimming in turbulence, I compared the statistics of flow properties likely to be sensed by larvae (fluid acceleration, deformation, vorticity, and angular acceleration) between diving and non-diving individuals. My analyses showed that diving larvae experienced high flow accelerations in short time intervals prior to dive onset, while accelerations experienced by non-diving larvae were significantly lower. Further, the probability that larvae dove increased with the fluid acceleration they experienced. These results indicate that oyster larvae actively respond to hydrodynamic signals in the local flow field, which has ecological implications for settlement and predator avoidance. 


\subsection{Light stimulates swimming behavior of larval eastern oys- ters (Crassostrea virginica) in turbulent flow}

In Chapter 5, I added an additional layer of complexity to the question of oyster larval responses in turbulence. Larvae experience a wide variety of physical, chemical, and biological water column cues during the settlement period, and likely experience none of these in isolation, which led me to examine the interactive effects of two water column cues on larval oyster settlement: light and turbulence. Using a grid-stirred tank and particle image velocimetry system as described in Chapters 2 and 3, larvae were exposed to a range of turbulence regimes in both light and dark conditions. In all turbulence levels, light had no effect on the proportion of larvae swimming upward, but had a modest effect on the probability of diving and helical swimming. Further, the geometry of helical swimming changed significantly between larvae swimming in light and dark conditions in conjunction with certain turbulence regimes, suggesting that the responses observed may be photokinetic or anti-predatory in nature.

\subsection{Larval oyster swimming responses to adult chemical exu- dates: evidence for the desperate larva hypothesis?}

In Chapter 6, I examined the effects of one of the most definitive settlement cues on oyster larval behavior. Competent-to-settle larval oysters respond to chemical exudates from adult oysters and their associated biofilms by increasing settlement, but how larvae actively modify their swimming behavior in response to said cues is not well understood. Further, larval responses may change with age, as old larvae may react to substandard settlement cues that newly competent larvae ignore (i.e., "the desperate larva hypothesis"). I exposed larval oysters to two seawater regimes: one regime had no settlement cue and the second had a cue derived from adult oyster exudates. Observations of larval swimming behavior were repeated throughout larval competency. Larvae exposed to the chemical cue demonstrated a distinctive downward swimming behavior throughout the competency period. In contrast, larvae exposed to no cue swam in an exploratory manner early in competency but exhibited

behaviors more similar to cue-exposed larvae as they aged. These results suggest that oyster larvae will ultimately settle for a poor settlement site to avoid death in the water column. 


\section{Chapter 2}

\section{Ontogenetic changes in larval}

\section{swimming and orientation of}

\section{pre-competent sea urchin Arbacia punctulata in turbulence}

\subsection{Abstract}

${ }^{1}$ Many marine organisms have complex life histories, having sessile adults and relying on the planktonic larvae for dispersal. Larvae swim and disperse in a complex fluid environment and the effect of ambient flow on larval behavior could in turn impact their survival and transport. However, to date, most studies on larval-flow interactions focus on competent larvae near settlement. We examined the importance of flow on early larval stages by studying how local flow and ontogeny influence swimming behavior in pre-competent larval sea urchins, Arbacia punctulata. We exposed larval urchins to grid-stirred turbulence and recorded their behavior at two stages (four and six-armed plutei) in three turbulence regimes. Using particle image velocimetry to quantify and subtract local flow, we tested the hypothesis that larvae respond to turbulence by increasing swimming speed, and that the increase varies with ontogeny. Swimming speed increased with turbulence for both four and six armed larvae, but their

\footnotetext{
${ }^{1}$ Currently in revision as "Wheeler JD, Chan KYK, Anderson EJ, and Mullineaux LS. Ontogenetic changes in larval swimming and orientation of pre-competent sea urchin Arbacia punctulata in turbulence", for Journal of Experimental Biology.
} 
responses differed in terms of vertical swimming velocity. Four armed larvae swam most strongly upward in the unforced flow regime, while six-armed larvae swam most strongly upward in weakly forced flow. Increased turbulence intensity also decreased the relative time that larvae spent in their typical upright orientation. Six-armed larvae were tilted more frequently in turbulence compared to four-armed larvae. This observation suggests that as larvae increase in size and add pairs of arms they are more likely to be passively re-oriented by moving water, rather than being stabilized by an increase in weight, potentially leading to differential transport. The positive relationship between swimming speed and larval orientation angle suggests that there was also an active response to tilting in turbulence. Our results highlight the importance of turbulence to planktonic larvae, not just during settlement, but also in earlier stages through morphology-flow interactions.

\subsection{Introduction}

Many marine invertebrates have complex life histories in which the planktonic larval phase acts as the vehicle to connect otherwise disjoint benthic adult populations which are mostly non-mobile $[42,13]$. Larval supply, both in terms of number of settlers and their conditions, plays an important role in determining population dynamics and community interactions [43, 44]. Larval swimming behaviors in response to various chemical, biological, and physical cues have important implications for the adult populations [45]. For planktonic, pre-competent larvae, swimming behaviors significantly impact vertical distribution patterns which in turn shape dispersal [46, 47]. For older, competent larvae, behaviors around settlement sites could significantly affect recruitment patterns $[48,49,50]$.

One such set of behavior-triggering cues are the hydromechanical signals associated with turbulent flow which larvae experience in nature [51,52]. These hydromechanical signals can be interpreted as three interacting components, namely acceleration, rotation, and deformation [53]. Larvae may respond to combinations of these signals in active and/or passive manners. In the water column, strong swimming larvae such as crab zoea actively change their swimming speeds in response to turbulence intensity [54] and barnacle cyprids swim upwards to counteract downwelling currents [55]. For moderate swimming larvae such as oyster larvae, a plasticity in response to turbulence has been observed, where competentto-settle larvae have been observed both to sink [27] and swim upward [56, 28] in high 
turbulence; these active behavioral responses may be regulated by body size. For weaker swimming plankton, such as larval sand dollars, it has been observed that their morphologies primarily interact passively with ambient flow $[57,46]$. Plutei of larval sand dollars represent an "armed morphology", for which organisms use ciliated extensions (such as arms or lobes) for feeding and swimming. This type of dual-use structure is shared between many weakly swimming marine invertebrate larvae, such as other echinoderms, molluscs, and some polychaete larvae [8]. Shuttlecock-like pluteus larvae have long ciliated arms supported by a calcite skeleton [58], and add pairs of arms (from 2-armed, to 4-armed, to 6-armed, etc.) through their development. Plutei are characterized by fore-aft asymmetry [57] and in some species, a distinct density differential in which mass is concentrated in the posterior [59]. Both these morphological characteristics of pluteus larvae induce passive reorientation in low Reynolds number, by two different mechanisms. Fore-aft asymmetry coupled with negative buoyancy produces a gravity-induced hydrodynamic torque [60], while a differential density produces a torque proportional to the distance between the larva's centre of gravity and centre of buoyancy $[59,60]$. Both mechanisms likely play a role in observed reorientation of larvae in flow, but previous experimental evidence suggests that urchin larvae primarily rely on non-homogeneous density, or "bottom heaviness" to reorient [59]. Larval-turbulence interaction is therefore a combination of both active behavioral choices such as those of barnacle cyprids and larval oysters and passive biomechanical limitations imposed by the pluteus morphologies of larval sand dollars and sea urchins.

The three way interactions between larval behavior, morphology, and the surrounding flow have significant implications for population distributions. Clay and Grünbaum [46] reported that 4 and 8-armed larval sand dollars are more likely than 6-armed larvae to be passively reoriented into downwelling regions by tilting in shear flow. This tilting in shear suggests an inability to maintain a stable orientation (hereafter stability) - the larva's usual vertically-directed swimming with arms in an upright position. Such ontogenetic differences in stability could imply distinct passive mechanisms to mediate transport and vertical position through ontogeny, and indeed, field observations of larval sand dollars suggest that older larvae are more likely to be found in deeper waters [61]. Swimming-flow interactions could also affect distribution patterns through aggregation. Some weakly swimming plankton, e.g. Heterosigma akashiwo, exhibit strong spatial and temporal patchiness driven by gyrotactic motility in shear: directed motion resulting from the orientation of cell axes 
through the balance of viscous and gravitational torque $[62,63,64]$. Therefore, quantifying swimming behaviors of planktonic larvae in environmentally relevant flow fields is essential for understanding transport and dispersal.

To date, however, most studies focus on larval-turbulence interactions on competent larvae preparing to settle (but see $[65,66,52]$ ). Various studies highlight that larvae actively respond to turbulence or components of turbulence, e.g., larval boat snails Crepidula fornicata increase upward swimming with increasing turbulence level [67], larval sea slugs Phestilla sibogae retract their vela when encountering turbulent filaments containing chemical cues [48], and larval eastern oysters Crassostrea virginica dive when experiencing high fluid acceleration over short time intervals [29]. Together with other modeling studies, these earlier works suggest turbulence enhances larval settlement [68, 56] (but see [69], who suggested that an increase in turbulence reduces settlement in scallop larvae). Recently, deformation associated with horizontal shear has been suggested to induce competency in larval urchins [70] and to induce cloning in coral larvae [71]. While there is increasing information about how late stage larvae respond to realistic flow fields near settlement, there is still relatively little understanding on how weakly swimming ciliated planktonic larvae respond to turbulence in earlier life stages and how that response may change through ontogeny.

In this study we exposed the weakly swimming planktonic larvae of the sea urchin Arbacia punculata, to three environmentally relevant turbulent flow regimes at two different developmental stages (4- and 6-armed plutei). We hypothesize that 1) larvae actively modify their swimming speeds in response to ambient flow conditions such that older, larger larvae swim faster and 2) larvae are passively reoriented in flow such that older, larger larvae are more stable due to bottom heaviness. Using non-invasive video tracking and flow subtraction techniques, we investigated the effect of turbulence on swimming speed and stability of larval A. punculata through ontogeny.

\subsection{Materials and Methods}

\subsubsection{Study organism and larval culturing}

The study organism is the purple urchin A. punculata, which has long been a focus species of embryonic and larval development studies [72,73] with well-established eco-toxicological responses [74]. Two male and two female A. punctulata adults were procured from the Marine 
Biological Laboratory Animal Supply (Woods Hole, MA, USA) and were injected with 0.55M $\mathrm{KCl}$ to induce spawning [75]. Oocytes collected were washed through a $63 \mu \mathrm{m}$ mesh to remove debris and sperm were collected dry. Eggs of each female were fertilized in $0.22 \mu \mathrm{m}$ filtered seawater $\left(\sim 18^{\circ} \mathrm{C}, 32 \mathrm{psu}\right)$ with sperm solutions from both males at concentrations

of approximately 1000 sperm $\mathrm{mL}^{-1}$. Fertilization success was visually confirmed by the presence of a fertilization envelope in $>95 \%$ of the eggs at 20 minutes post-fertilization. Maternal cultures were reared separately and later combined in equal proportions for the experimental observations.

At 24 hours post-fertilization, hatched embryos from each female were transferred into six 16 L plastic containers (12 containers total) holding filtered seawater $(0.22 \mu \mathrm{m}$ filtered, $32 \mathrm{psu})$ at a density of 6 individuals $\mathrm{mL}^{-1}$. This rearing density was chosen to provide a sufficient number of larvae for subsequent video observations. These containers were aerated and kept in an environmental chamber maintained at $18^{\circ} \mathrm{C} \pm 1^{\circ} \mathrm{C}$. Larvae were fed ad lib

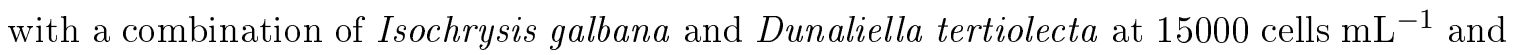
2500 cells $\mathrm{mL}^{-1}$ daily. Complete water changes were performed every 3 days. Daily $20 \mathrm{~mL}$ subsamples were taken from each container and examined under a microscope to confirm normal larval development.

\subsubsection{Video observations of larval swimming in turbulence}

Swimming behaviors of larvae in different levels of turbulence were observed at 8 and 23 days post-fertilization (4 and 6-armed larval stages, respectively). These observations were made in a Plexiglas tank $(44.5 \times 44.5 \times 90 \mathrm{~cm})$ equipped with two vertically-oscillating grids identical to that detailed in [28]. Larval swimming and fluid motion were observed in a vertical cross-section field of view (FOV) of $3.5 \mathrm{~cm} \times 3.5 \mathrm{~cm}$ in the centre of the tank using a monochrome high speed video camera (Photron Fastcam SA3) at 60 frames per second (fps). The FOV was illuminated with a sheet of light from a near-infrared laser (Oxford Lasers, Firefly 300 W, $1000 \mathrm{~Hz}, 808 \mathrm{~nm}$ ) set perpendicular to the camera line of sight.

Four replicate trials were conducted for each stage using different batches of larvae, with the tank drained and rinsed between trials. Larvae were subsampled from all culturing vessels, i.e., larvae from both mothers were simultaneously used for these experimental trials. Larval urchins were gently poured into the tank from a beaker, with approximately 60,000 and 28,000 larvae introduced per trial at day 8 and day 23, respectively, for larval densities 
of $<0.5$ individual $\mathrm{mL}^{-1}$. Neutrally buoyant polystyrene particles of 3 to $3.4 \mu \mathrm{m}$ diameter (Spherotech, $2.5 \mathrm{~cm}^{3}$ of a $10 \% \mathrm{w} / \mathrm{v}$ suspension) were injected into the tank as passive tracer particles for particle image velocimetry (PIV).

During each trial, larval urchins were exposed to three different treatment levels by oscillating the grids at $0,0.25,0.5 \mathrm{~Hz}$ at a fixed amplitude of $5 \mathrm{~cm}$. The energy dissipation rates of these three regimes were previously computed using a separate set of PIV experiments and the resultant 2D flow vectors as per Doron et al. (2001), and were estimated to be 0 , 0.002 , and $0.017 \mathrm{~cm}^{2} \mathrm{~s}^{-3}$ [28]. Kolmogorov length scales for the two higher flow regimes were 0.147 and $0.088 \mathrm{~cm}$, respectively, and the integral length scales were 3.023 and $3.649 \mathrm{~cm}$, respectively, corresponding to the ranges of smallest to dominant energy-containing eddies in the tank [29]. The mean 4- and 6-armed larval midline lengths, for comparison, were approximately 0.013 and $0.018 \mathrm{~cm}$ respectively. Hereafter these three turbulence treatments are referred to as unforced, low forcing, and moderate forcing. The qualitative terms are intended to describe the relative intensity of the turbulence in the range of what larvae might experience in field conditions. While surf zone conditions can reach energy dissipation rates of $10^{0}$ to $10^{4} \mathrm{~cm}^{2} \mathrm{~s}^{-3}$ [70], tidal and estuarine flows are calmer $\left(10^{-2}\right.$ to $\left.10^{0} \mathrm{~cm}^{2} \mathrm{~s}^{-3}\right)$ with energy dissipation rates decreasing further away from the coast [76]. Our turbulence forcing regimes reflect energy dissipation rates of the open ocean and of calmer nearshore waters. Larvae were exposed to increasing turbulence from unforced flow to the moderate forcing in a sequential manner. Five minutes of spin up time at the beginning of each turbulence level allowed the flow to equilibrate before filming, and multiple 45 second video clips ( $5-11$ clips) were collected at each turbulence level. Video clips were collected until an adequate total number of larvae $(>100)$ had been observed in the FOV. In between treatments of low and moderate forcing, the grids were stopped to allow flows to dissipate. Additional unforced flow video clips were collected during this interval after the transient net downward flow was no longer visible in the FOV. All video clips were exported and saved as high-resolution $(1024 \times 1024$ pixels $)$ TIFF images and used for larval tracking and flow visualization.

At the end of each trial, larval urchins were collected on a $100 \mu \mathrm{m}$ mesh and fixed in buffered $2 \%$ paraformaldehyde for later microscopy and image analysis. Body lengths, lengths of skeletal arm rods and stomach were measured using the software Fiji (Fig. 2-1, [77]). 


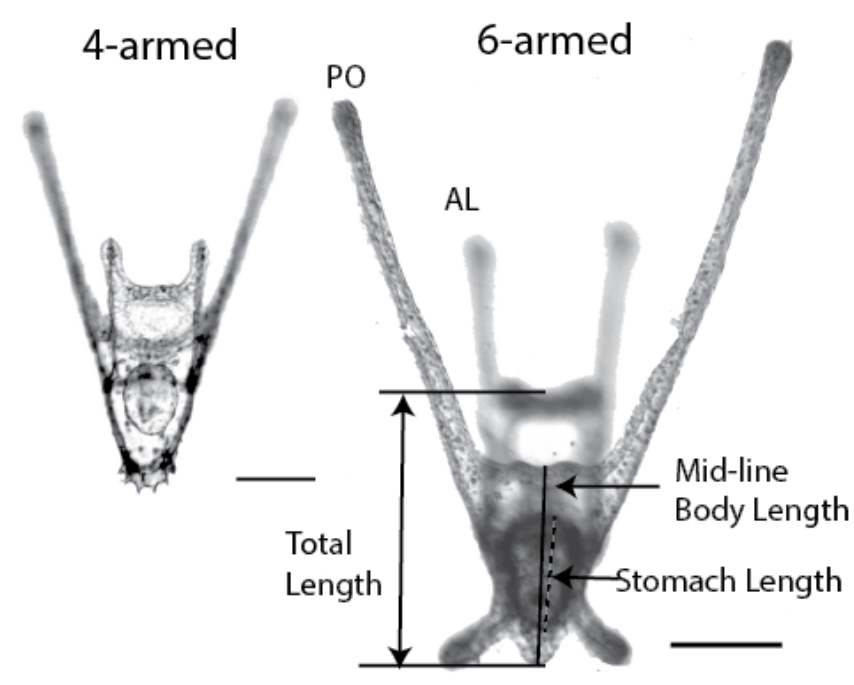

Figure 2-1: A comparison of the relative size and morphologies of the four-armed larva (left) and six-armed larva (right), with a $100 \mu \mathrm{m}$ bar for scale (lower right). The labels 'PO' and 'AL' indicate postoral and anterolateral arm, respectively. Reported length scales are stomach length, mid-line body length, and total length (where total length is defined from the base of the body to the oral hood, neglecting arm length).

\subsubsection{Larval tracking}

Observed larval movement is a combination of both individual swimming motion and advection by surrounding fluid, so we adapted the flow subtraction method from $[28,29]$ to test if swimming behaviors alone vary with changes in flow conditions. To compute larval velocities in such a relative framework (isolated from advection in flow), we first identified larval positions in each frame and calculated absolute velocity of individuals in the flow field. We subsequently estimated local flow velocities using PIV and subtracted these to isolate larval swimming velocities from advection.

The flow velocities were calculated using LaVision DaVis Imaging Software (v. 7.9). Each high resolution TIFF image (for example Fig. 2-2 A) was subdivided into $16 \times 16$ pixel interrogation windows with no overlap and the average displacement of particles in each window between images was determined by a multi-pass 2D FFT (Fast Fourier Transform) analysis scheme. Velocity fields were converted into MATLAB data files for use in the subsequent flow subtraction, and smoothed with a 10-time step interval boxcar filter to eradicate high frequency noise. Full details of the PIV procedure are presented in [28]. 

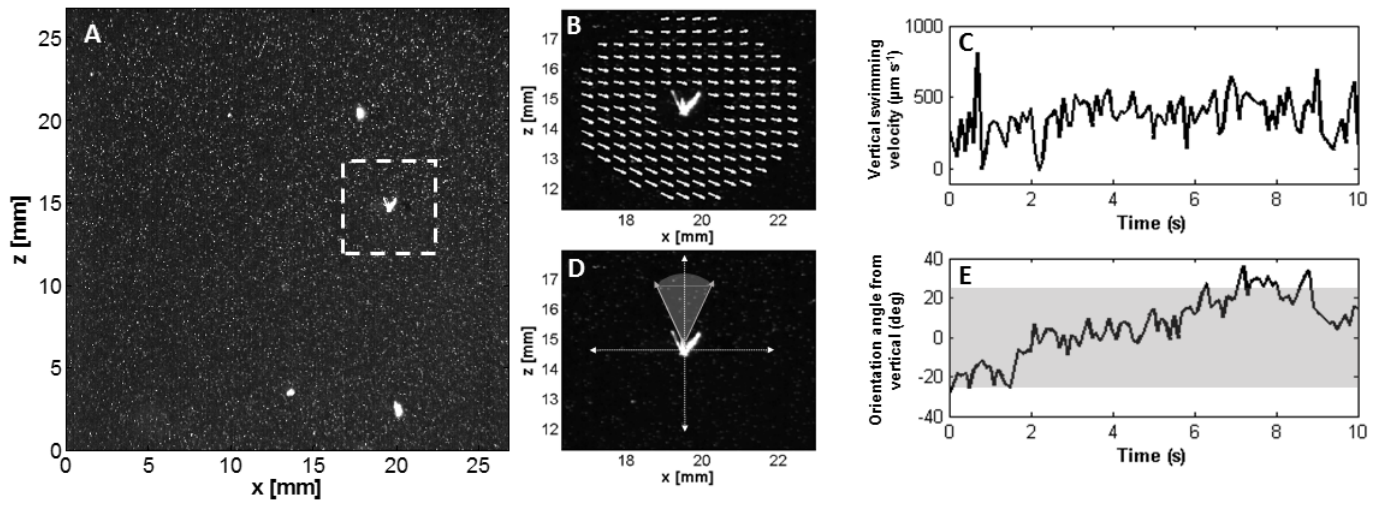

Figure 2-2: A) Sample experimental image, where in-focus larva (with arms clearly visible) is highlighted in white dashed box. Smaller white specks are passive particles and larger diffuse spots are out of focus larvae. (B) Close-up of highlighted larva, with overlaid PIV velocity field (white arrows) surrounding larva. Passive particle intensity is dimmed for clarity. (C) Sample time series of an individual larva's vertical swimming velocity as it was tracked in the field of view. (D) Close-up of highlighted larva, with overlaid Cartesian coordinate system and shaded range of angles $\left(-25^{\circ}\right.$ to $25^{\circ}$ from vertical) at which larva is considered upright. (E) Sample time series of an individual larva's orientation angle from vertical as it was tracked in the field of view. The shaded region denotes the range of orientation angles at which the larva is considered upright.

To track individual larvae, the TIFF images were filtered to remove background noise and thresholded for brightness, leaving larvae to appear as white silhouettes on a black background. The centroid position of individual larvae was recorded frame by frame using customized LabView 2010 (National Instruments) software. Larval tracks were then compiled with in-house MATLAB (v.7.12.0 R2011a) software by connecting centroids frame-by-frame within a user-defined threshold distance, and used to calculate larval velocity in the FOV.

\subsubsection{Flow subtraction and larval velocities computation}

Larval positional data were integrated with PIV flow field data in MATLAB, where an annulus of flow velocity vectors around each larva was identified in each frame (Fig. 2-2 B). The annulus had an inner radius equal to the sum of the larval radius and the PIV spatial grid interval, to mask poor PIV velocity estimates near the larval position. The outer radius was 4 times the inner radius, to incorporate $\sim 4-6$ velocity vectors away from the larva in all directions. The flow velocity at the larval position was then estimated: a second order two-dimensional Taylor expansion was fit to the annulus velocity vectors and interpolated to 
the larval position. Larval swimming velocities (independent of advection in the flow) were calculated at each time step (Fig. 2-2 C) by subtracting the flow velocity (interpolated to the larval position) from the absolute velocity . For each larva, we denote swimming speed at each time step as $\mathbf{V}_{\mathbf{s}}=\left[u_{s}, w_{s}\right]$, where $\mathbf{V}_{\mathbf{s}}$ is overall swimming velocity vector, $V_{s}$ is the vector magnitude, or speed, and $u_{s}, w_{s}$ are horizontal and vertical velocities respectively.

\subsubsection{Larval orientation}

To identify larval orientation, TIFF images were imported into MATLAB and subsampled in time to $10 \mathrm{fps}$. A subset of larvae was randomly selected (per video clip), and from larval position data these larvae were magnified individually to facilitate the identification of the larval orientation axis. The larval orientation axis was determined by extending the body midline to a point equidistant between the visible pair of arms (anterolateral or posterodorsal), and this process was repeated frame-by-frame to construct a time series for the orientation angle of each larva. A total of 16 larval trajectories (sampled at $10 \mathrm{fps}$ ) in each turbulence regime (3 levels) for each of the 4 trials were analyzed in this way, for a total of 192 larval trajectories in each of the 4 and 6-armed groups. We computed the angular deviation between orientation axis and the true Cartesian vertical (defined here as $0^{\circ}$, using the FOV as the reference), hereafter referred to as larval orientation angle (Fig. 2-2 D). Using the orientation time series of each larva (Fig. 2-2 E), we computed the proportion of time spent upright by normalizing the duration in which orientation angle lies between $\pm 25^{\circ}$ from the vertical, with the total time the larva was tracked. The identification of orientation axes was not automated due to the highly variable appearance of the multi-armed larvae in two dimensions. Attempts to automate orientation computations led to heavy biases toward the angles of the arms that were closest to the focal plane, which were much brighter than the more distant arms. While the range of angles from vertical defining an upright orientation $\left( \pm 25^{\circ}\right)$ was chosen somewhat arbitrarily, analysis with various magnitudes of deviation showed that our subsequent statistical conclusion is not overly sensitive to this parameter.

\subsubsection{Statistical analysis}

We compared changes in larval size between 4- and 6-armed stages using a $t$-test. We tested for the effect of turbulence level on vertical and horizontal swimming velocities, swimming 
speed, and stability using non-parametric statistical methods because the dataset did not meet the assumption of equal variance. We compared the swimming speeds $\left(V_{s}\right)$ and velocities $\left(u_{s}, w_{s}\right)$ across all forcing regimes between the two stages with a Mann-Whitney test. We also compared the effect of the turbulence regime on the distributions of horizontal velocity $\left(u_{s}\right)$, vertical velocity $\left(w_{s}\right)$, and speed $\left(V_{s}\right)$ within the 4 - and 6-armed stages separately with Kruskal-Wallis tests. We compared the effect of turbulence regime on stability at each of the two developmental stages using the proportion of time spent upright as a metric with Kruskal-Wallis tests, and identified possible directional biases in orientation angle with a Wilcoxon signed-rank test. We further explored the relationship between orientation angle and horizontal velocity $\left(u_{s}\right)$, vertical velocity $\left(w_{s}\right)$ and swimming speed $\left(V_{s}\right)$ with a Spearman rank correlation for each of the developmental stages.

\subsection{Results}

\subsubsection{Larval size and swimming speed increased with ontogeny}

As larval urchins developed, their overall size significantly increased, with an average midline body length of $131.0 \pm 10.1 \mu \mathrm{m}$ at the 4-armed stage and $175.8 \pm 5.5 \mu \mathrm{m}$ at the 6 -armed stage ( $t$-test, $F=135.1, p<0.001$; Table S1). Considering all turbulence regimes combined, swimming speed $\left(V_{s}\right)$ increased through ontogeny, with an average flow-subtracted swimming speed of $748.6 \pm 14.6 \mu \mathrm{m} \mathrm{s}^{-1}$ at the 4 -armed stage and $953.5 \pm 18.9 \mu \mathrm{m} \mathrm{s}^{-1}$ at the 6 -armed stage (Mann Whitney $U=1921191, p<0.0001$ ). However, vertical and horizontal velocities varied with stage in a different pattern, such that there was no significant effect of stage on horizontal velocity but a significant effect on vertical velocity (Mann Whitney $U=1212659$, $p<0.0001)$.

\subsubsection{Swimming speeds changed with turbulence level but differed be- tween stages}

Generally, larval urchins swam in relatively straight, upwardly directed paths. Their swimming speeds varied significantly between turbulence regimes at both 4 -armed ( $K=438.8, p$ $<0.001$, Fig. 2-3 A) and 6-armed stages $(K=542.1, p<0.001$, Fig. 2-3 B), where overall speed $\left(V_{s}\right)$, increased monotonically with increasing turbulence intensity for both stages. The turbulence regime had a significant effect on vertical velocity $\left(w_{s}\right)$ for both 4 -armed 
$(K=6.446, p=0.040$, Fig. $2-3 \mathrm{C})$ and 6 -armed stages $(K=21.763, p<0.001$, Fig. 2-3 D). However, the two developmental stages demonstrated distinct relationships between turbulence and vertical swimming velocity: at the 4-armed stage, vertical velocity decreased slightly with increasing turbulence (Fig. 2-3 C) such that vertical velocity was fastest in the unforced regime with a median vertical velocity of $445 \mu \mathrm{m} \mathrm{s}^{-1}$. At the 6 -armed stage, vertical velocity increased and then decreased with increasing turbulence (Fig. 2-3 D), such that vertical velocity was highest in the low forcing regime with a median vertical velocities of $337 \mu \mathrm{m} \mathrm{s}^{-1}$, compared to $244 \mu \mathrm{m} \mathrm{s}^{-1}$ in the unforced regime and $122 \mu \mathrm{m} \mathrm{s}^{-1}$ at moderate forcing. Larval horizontal velocities $\left(u_{s}\right)$, however, did not significantly change with turbulence at either the 4 -armed $(K=4.896, p=0.086)$ or 6 -armed stages $(K=0.784$, $p=0.676)$.

\subsubsection{Stability decreased with turbulence and age}

Larval orientation angle in all observed cases averaged close to vertical $\left(0.9^{\circ} \pm 16.5^{\circ}\right)$, with $>73 \%$ of all observations within $\pm 10^{\circ}$. The median angle did not significantly differ from zero $(W=-472, p=0.914)$, demonstrating that the larvae as a population had no preferred direction (positive or negative) in orientation angle from vertical. As expected, turbulence did not bias larval orientation angle, as the flow was near isotropic and homogeneous. The distribution of median angle was unimodal, demonstrating that there were not multiple groups of larvae with preferred directional angles which averaged to zero. However, transient tilting occurred frequently in turbulence. Larvae at both developmental stages spent over 95\% time in the upright position in the unforced regime (Fig. 2-3 E-F), but time spent upright decreased significantly with turbulence for both 4 -armed $(K=17.33, p<0.001$, Fig. 2-3 E) and 6-armed larvae $(K=27.62, p<0.001$, Fig. 2-3 F). Older larvae appeared more susceptible to tilting than younger larvae: in the moderate forcing regime, the younger 4-armed larvae were upright on average $87.6 \%$ (S.E. $\pm 2.9 \%$ ) of the observed time compared to $69.7 \%$ (S.E. $\pm 4.1 \%$ ) at the 6 -armed stage. 

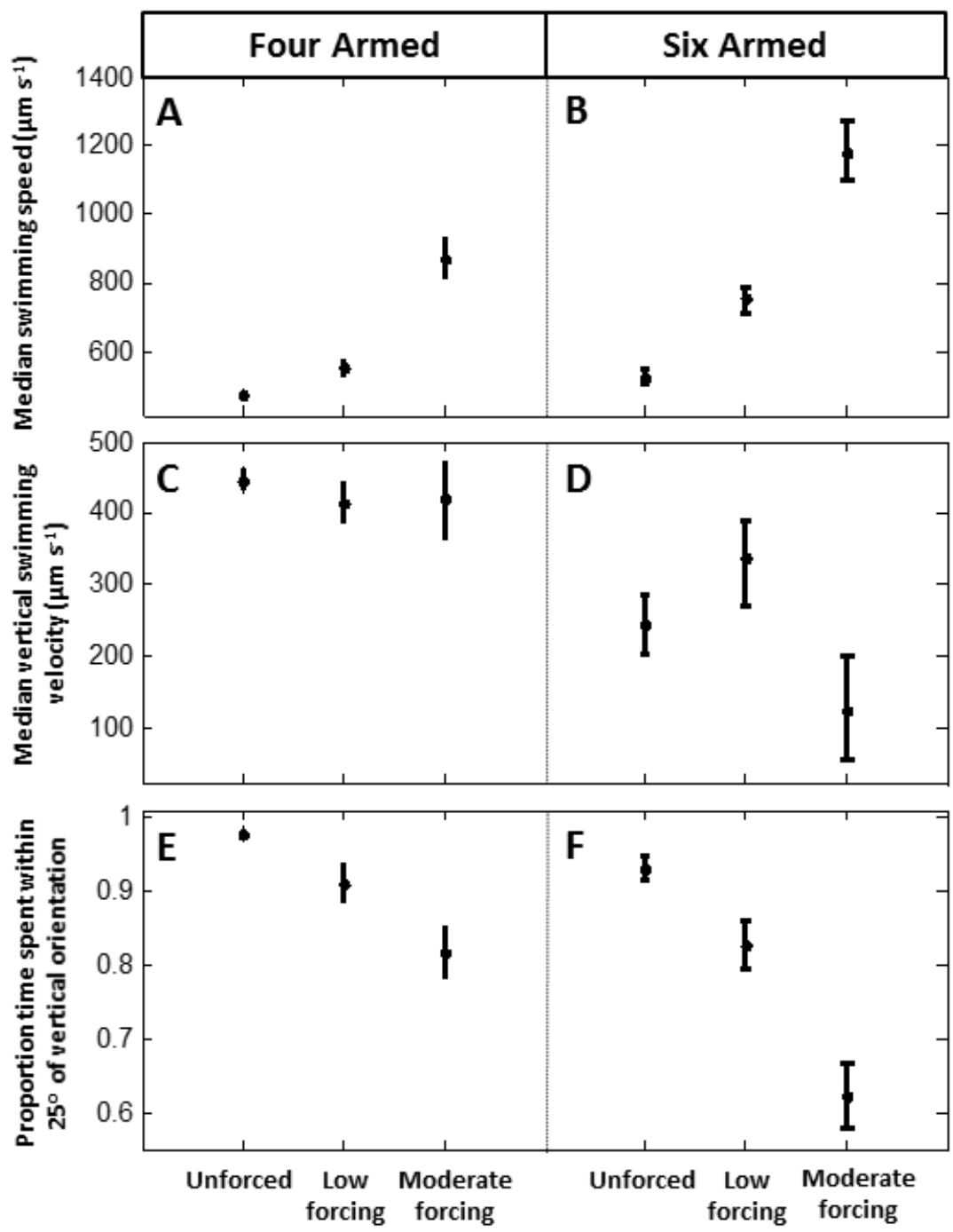

Figure 2-3: (A-B) Median larval swimming speed with 95\% confidence intervals, with respect to turbulence regime, for four armed larvae (A) and six armed larvae (B). (C-D) Median larval vertical swimming velocity with $95 \%$ confidence intervals, with respect to turbulence regime, for four armed larvae (C) and six armed larvae (D). (E-F) Mean proportion of time spent by larvae within $25^{\circ}$ of the vertical orientation with $95 \%$ confidence intervals, with respect to turbulence regime for four armed larvae $(\mathrm{E})$ and six armed larvae $(\mathrm{F})$.

\subsubsection{Swimming speed correlated with orientation angle}

We explored the relationship between larval orientation (measured from the vertical, $0^{\circ}$ ) and swimming speeds across all flow regimes and found a considerable difference between the 4 and 6-armed stages. At the 4-armed stage, there was no significant relationship between the orientation angle and overall swimming speed, horizontal, or vertical velocity $(p>0.126)$. 
In contrast, at the 6-armed stage, the slight deviations from vertical orientation (Fig. 2-4 A, data points at lower left) were significantly correlated with increased swimming speed (Spearman rank $\rho=0.163, p=0.002$, Fig. 2-4 A). There was no significant correlation between horizontal velocity and orientation angle (Spearman rank $\rho=0.0716, p=0.325)$. Vertical velocity, however, was negatively correlated with orientation angle (Spearman rank $\rho=-0.221, p=0.002$, Fig. $2-4$ B), i.e. tilted larvae were more likely to sink than upright larvae. The sinking of larvae due to negative buoyancy explains how it is possible to have negative vertical velocities even though orientation angles are less than $90^{\circ}$ (Fig. 2-4 B). Observed variance in swimming speed is due in part to the range in body size of observed larvae (in addition to behavioral components) and the variance may partially obscure the changes in larval behavior when tilted. Probability distributions of swimming speed (Fig. 2-4 C-D) and vertical velocity (Fig. 2-4 E-F) for upright versus tilted larvae highlight the changes in behavior: median swimming speed in upright larvae is $624.1 \pm 34.8 \mu \mathrm{m} \mathrm{s}^{-1}$ (Fig. 2-4 C) and increases to $817.3 \pm 116.5 \mu \mathrm{m} \mathrm{s}^{-1}$ in tilted larvae (Fig. 2-4 D). Upright larvae have a median vertical velocity of $346.5 \pm 31.3 \mu \mathrm{m} \mathrm{s}^{-1}$ (Fig. 2-4 E) while tilted larvae have a median vertical velocity of $91.2 \pm 87.6 \mu \mathrm{m} \mathrm{s}^{-1}$ (Fig. 2-4 F). 

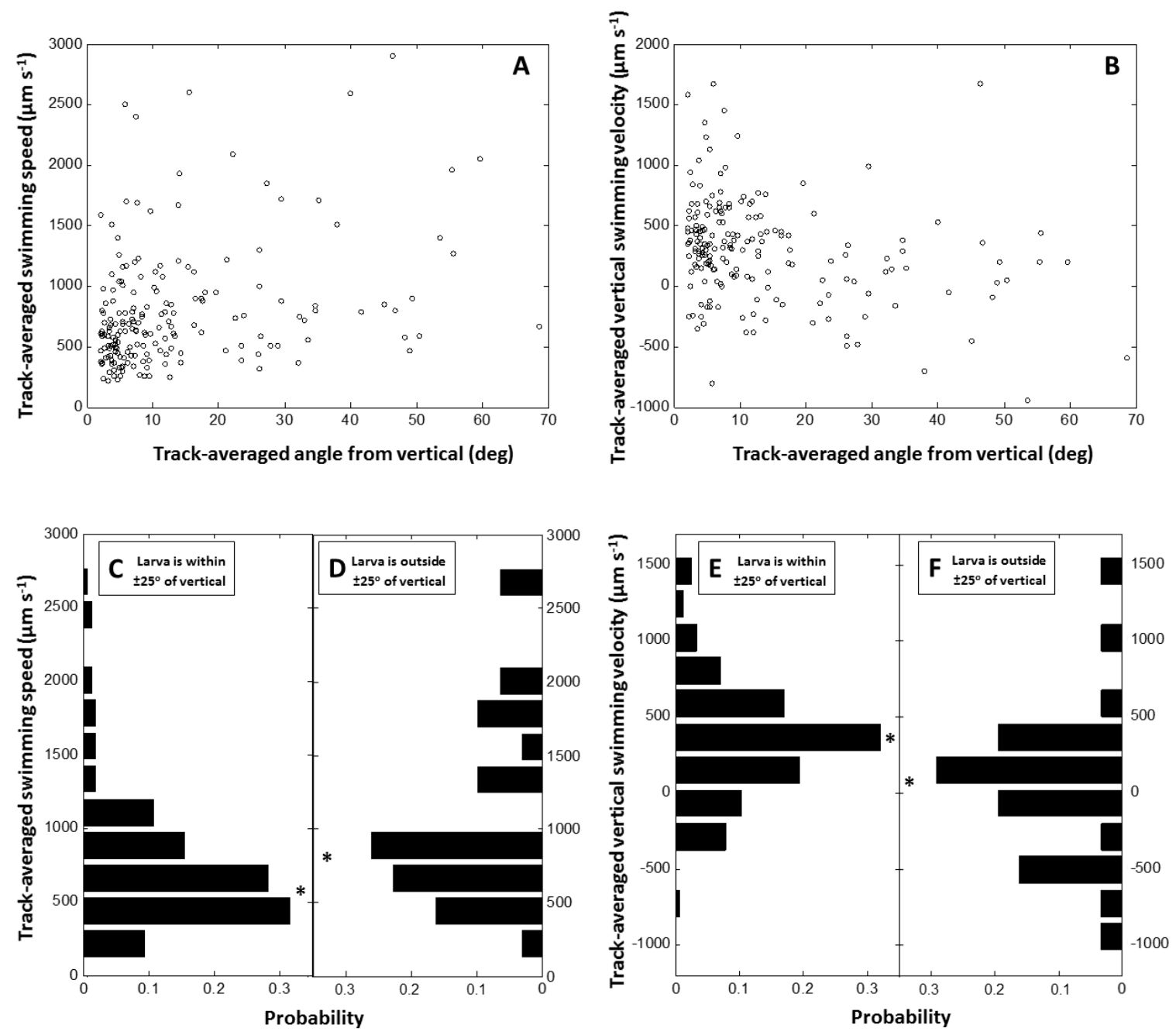

Figure 2-4: (A-B) Track-averaged orientation angle ( $0^{\circ}$ is vertical) versus track-averaged swimming speed (A) and track-averaged vertical swimming velocity (B). Each point represents an individual six-armed larva, using larvae from all turbulence regimes. (C-F) Probability distribution of track-averaged swimming speed in upright larvae (C) versus tilted larvae (D), and probability distribution of track-averaged vertical swimming velocity in upright larvae (E) versus tilted larvae $(\mathrm{F})$. Asterisks denote median speed or velocity for each distribution. Note that the $x$-axes for the tilted larvae (D and $\mathrm{F}$ ) are reversed, so that they are mirror images of $\mathrm{C}$ and $\mathrm{E}$, respectively. 


\subsection{Discussion}

Marine invertebrate larvae swim in a complex fluid environment, and their responses to hydromechanical signals during their planktonic and near-settlement stages have significant implications for transport, survival, and recruitment. By exposing two different stages of larval urchin A. punctulata to different turbulence regimes in the laboratory, we found that larval swimming speed increased and vertical velocities changed with increasing turbulence. Vertical velocity of the older 6-armed larvae increased at low forcing but decreased when experiencing moderate forcing, suggesting more downward movement. This is consistent with our observations that 6-armed larvae were more unstable when exposed to turbulence and spent less time in the upright position compared to 4-armed larvae. Such changes in the stability through ontogeny can potentially lead to changes in depth distributions as larvae age: larvae may occupy wider or narrower bands of depth, or shift from surface to deeper water. Such changes to vertical distributions may in turn impact larval survival and transport. Swimming speed and vertical velocity of larval urchins changed significantly with age and turbulence regime (Fig. 2-3). The older, larger 6-armed larval urchins had higher swimming speeds than 4-armed larvae, and this increase is consistent with earlier hydromechanical model predictions that weight carrying capacity is proportional to total arm length [78]. Here, weight carrying capacity is defined as the maximum net downward force, i.e., the difference between gravitational pull and buoyancy sustained by a larva at zero velocity. However, this increase in swimming speed with turbulence did not translate to an increase in vertical velocity. For 4 -armed larvae, the median vertical velocity of the population was smaller in both the forced regimes compared to the unforced regime. For 6-armed larvae, median vertical velocity presented a dome shaped response with increasing turbulence, such that vertical velocity was the largest at low forcing and smallest at moderate forcing. Larval speed, velocity, and orientation angle are all related $\left(u_{s}=U_{s}(t) \sin (\theta(t))\right.$ and $w_{s}=U_{s}(t) \cos (\theta(t))$, where $\theta$ is the orientation angle), where these relationships hold instantaneously in time. Our decision to average these terms separately over larval trajectories implies, however, that direct relationships between track-averaged speed, velocity, and orientation angle with respect to turbulence regime may be confounded by perturbation covariance terms. The relative strength of mean to perturbation terms under the trajectory averaging operator is the subject of future investigation. 
Contrary to other marine invertebrate larvae, e.g. mollusc veligers [26, 28], vertical velocity of larval urchins did not increase with increased turbulence. However, these results are consistent with previous observations of other echinoderms through ontogeny: older larval sand dollars and larval green urchins have a lower vertical velocity under no flow or shear conditions compared to younger larvae [65, 79].

The observed differences in vertical velocity between 4- and 6-armed larvae may stem from a changing morphology as they age. While the increased number and length of arms could provide more weight carrying capacity [78], the associated increase in the calcite skeleton structure could potentially counteract this increase in weight carrying capacity and incur metabolic costs for upward swimming while carrying additional weight [58]. The importance of this second factor is supported by our observations of lower vertical swimming velocities in 6-armed larvae when compared to 4-armed larvae, across all turbulence regimes. However, since the functional response of vertical swimming velocity to turbulence regime also differed between the two developmental stages, we conclude that the ambient flow must also play a role, and that observed changes in swimming velocity are not solely driven by the balance between weight carrying capacity and size. Some potential explanations for the between stage differences are structural: the additional pair of posteriodorsal arms in the 6-armed larvae have fenestrated skeletal rods, providing drag to reduce passive sinking speeds [80], and these arms are connected to the larval body by muscles which allow larvae to orient these arms to redirect local flow fields or permit reverse swimming [81]. The ability to increase drag and redirect flow might help 6-armed larval urchins to a certain degree maintain their vertical velocities at low forcing, in comparison to unforced or moderate forcing regimes, where they may not engage these strategies.

Like swimming velocity, larval orientation also changed significantly with age and turbulence regime. Larvae of both age groups spent significantly less time upright as turbulence intensity increased, likely due to increased shear, and 4-armed larvae were significantly more stable than 6-armed larvae in all turbulence regimes. Ontogenetic changes in larval shape may play a role in the larval ability to maintain an upright orientation, and hence, stability in flow. Older, heavier larval urchins have increased skeleton weight as ballast which could enhance stability [78, 59]. However, the additions of arms and increased size could also compromise stability by affecting the separation distance between the center of gravity and center of buoyancy [78]. Individuals with longer or a wider spread of arms might also more 
readily cross stream lines, thus experiencing a larger fluid torque $[57,46]$. These biomechanical constraints may help account for the observation that 6-armed larvae were more prone to tilting in higher turbulence compared to smaller 4-armed larvae.

Our results highlight the importance of morphology in creating biomechanical constraints for movement. The pluteus morphology of echinoids varies significantly between families, but different morphological structures could serve a similar purpose: for example, the arbaciids studied here have posterolateral arms, while eucidaris have long basal rods. In both cases, and these extensions could potentially act to counter balance larvae in moving water. Computational fluid dynamics modeling work on functional morphology and associated tradeoffs of "armed" larvae would significantly contribute our understanding towards the ecology and evolution of larval forms [57, 82, 83].

Our observations suggest that pre-competent larval urchins respond to turbulence with both active and passive mechanisms: they actively increase their swimming speed in increased turbulence and are passively reoriented through morphology-flow interactions, which compromise their ability to maintain directed swimming. When exposed to increased turbulence, 6-armed larvae had higher swimming speeds but lower vertical swimming velocities than 4-armed larvae. It is important to note that a decrease in such time-averaged vertical velocity in the 6-armed larvae does not necessary represent a reduction in movement with turbulence. Instead, this change may reflect an increase in the proportion of time spent swimming in directions other than the preferred vertical orientation, suggesting a passive response to turbulence. This is supported by the significant negative correlation between orientation angle and vertical swimming velocities. However, the observed increase in swimming speed in higher turbulence regimes demonstrates that larvae are not merely being reoriented and persisting in a default swimming mode: the positive correlation between orientation angle and swimming speed demonstrates that larvae swim faster when they are tilted, or potentially that stronger swimmers are more prone to tilting.

The first interpretation suggests an intriguing possibility that an active behavior is triggered by a passive larval response to local flow conditions: while tilting and reorientation are likely passive responses to increased shear in turbulence, an increased swimming speed subsequent to tilting is an active response. Larval responses to ambient flow may involve more such interactions between active and passive responses than has previously been investigated. Despite the absence of a statocyst-like structure, our results support early observations that 
larval urchins have mechanoreception ability and are capable of adjusting their propulsive behaviors accordingly $[59,84,85]$. Further work on ciliary motion control $[86,87]$ and gene expression (e.g., orthologs of vertebrate mechanosensory genes, e.g. Sp-Usherin and TRPA1 $[88,89])$ could help understand the biophysical mechanisms of behavioral control under natural flow conditions.

The second interpretation, that faster swimming larvae are more prone to tilting, also has a sound biomechanical basis. Swimming larvae generate self-induced flows which decay away from the larval body. If these self-induced flows have greater energy dissipation rates than the background turbulence flow, then larvae are "shielded" from background turbulence in a local volume, the radius of which depends on how rapidly the self-induced flow decays. Gallager demonstrated that faster swimming bivalve larvae have steeper rates of decay of self-induced flows [90], suggesting that these larvae are more likely to be affected by background turbulence because of their smaller shielded volumes. This is consistent with our results, where faster swimming larvae are linked to decreased stability in turbulence. Future investigations of larval swimming in moderate and high turbulence regimes where tilting is most prevalent will help to further elucidate the relationship between orientation angle and swimming speed, and to determine larval response times for an active swimming response to tilting.

The observed changes in response to turbulence intensity through larval development have significant implications for larval distribution. If our observations in the laboratory can translate to the water column in the coastal ocean, older, 6-armed larvae would be more likely to be tilted, have slower vertical velocity and be downwardly transported in these energetic environments [46]. This differential swimming could result in selective transport, leading to ontogenetic differences in depth distribution $[79,78]$. One possible scenario is that the differential transport helps younger individuals to maintain themselves in the surface water where food abundance is higher and help older individuals to approach their settlement sites. Alternatively, differences in response and distribution could reflect different strategies to avoid predation. One possible hypothesis is that smaller, 4-arm larvae are less vulnerable to predators relying on vision, and hence, can survive even in well illuminated surface waters. Our results suggest that the observation of early stage pre-competent larvae in realistic flow conditions, in addition to competent larvae seeking settlement sites, is important for understanding the population dynamics of marine invertebrates. Our observations also 
demonstrated that a gyrotaxis-like reorientation response to turbulence is present in multicellular swimming plankton. Turbulence, therefore, not only impacts larval transport but could potentially shape the aggregation and distribution of other zooplankton and thereby impact ecological interactions through the modulation of patch dynamics.

\subsection{Acknowledgements}

We thank Susan Mills for larval culturing and experimental assistance, and Karl Helfrich for assistance with video set-up. We are grateful for the valuable discussion on data analysis and interpretation that Victoria Starczak and Jesús Pineda kindly provided. We also thank the facilities engineers, especially Richard Galat, at WHOI for their assistance in experimental setup. This work was supported by the National Science Foundation [OCE-0850419] and the National Oceanic and Atmospheric Administration Sea Grant [NA14OAR4170074]. K. Chan was supported by the Postdoctoral Scholar Program at the Woods Hole Oceanographic

Institution (WHOI), with funding provided by the Coastal Ocean Institute, the Croucher Foundation, and the Royal Swedish Academy of Sciences. K. Chan is currently funded by the Croucher Foundation. Additional funding was provided to L. Mullineaux through the WHOI Ocean Life Fellowship and discretionary WHOI funds, and to E. Anderson through the faculty sabbatical program at Grove City College. 


\subsection{Supplemental Material}

\begin{tabular}{|c|c|c|c|c|c|c|c|}
\hline & & & $\begin{array}{l}\text { Mid line } \\
\text { body length }\end{array}$ & Total length & $\begin{array}{l}\text { Post-oral } \\
\text { arm length }\end{array}$ & $\begin{array}{l}\text { Anterolateral } \\
\text { arm length }\end{array}$ & $\begin{array}{l}\text { Stomach } \\
\text { length }\end{array}$ \\
\hline \multirow[t]{3}{*}{ 4-armed } & \multirow[t]{3}{*}{ All trials } & Mean & 131.0 & 203.7 & 401.3 & 293.4 & 65.2 \\
\hline & & S.D. & 10.1 & 8.0 & 25.4 & 15.1 & 5.9 \\
\hline & & $\mathrm{n}$ & 18 & 18 & 18 & 18 & 18 \\
\hline \multirow[t]{15}{*}{6 -armed } & \multirow[t]{3}{*}{ Trial 1} & Mean & 185.0 & 262.9 & 561.4 & 371.7 & 96.9 \\
\hline & & S.D & 13.5 & 21.3 & 98.3 & 37.3 & 11.8 \\
\hline & & $\mathrm{n}$ & 16 & 16 & 16 & 16 & 16 \\
\hline & \multirow[t]{3}{*}{ Trial 2} & Mean & 186.3 & 263.2 & 574.1 & 383.2 & 105.7 \\
\hline & & S.D & 11.7 & 23.1 & 91.2 & 59.2 & 10.7 \\
\hline & & $\mathrm{n}$ & 17 & 17 & 17 & 17 & 17 \\
\hline & \multirow[t]{3}{*}{ Trial 3} & Mean & 166.1 & 238.8 & 546.1 & 337.6 & 93.5 \\
\hline & & S.D & 13.5 & 18.2 & 104.6 & 44.8 & 10.2 \\
\hline & & $\mathrm{n}$ & 18 & 18 & 18 & 18 & 18 \\
\hline & \multirow[t]{3}{*}{ Trial 4} & Mean & 167.6 & 237.7 & 540.5 & 344.7 & 93.5 \\
\hline & & S.D & 11.3 & 19.5 & 74 & 54.3 & 9.9 \\
\hline & & $\mathrm{n}$ & 18 & 18 & 18 & 18 & 18 \\
\hline & \multirow[t]{3}{*}{ All trials } & Mean & 175.84 & 250.09 & 555.09 & 358.63 & 97.29 \\
\hline & & S.D & 15.1 & 23.2 & 90.2 & 55.7 & 11.6 \\
\hline & & $\mathrm{n}$ & 69 & 69 & 69 & 69 & 69 \\
\hline
\end{tabular}

Table S1: Morphometrics of a sub-sample of larval urchins collected after the video observations, with length scales as defined in Figure 1. Note that individuals measured for not necessarily the same individuals observed in the videos. All the lengths are measured in units of $\mu \mathrm{m}$. 


\section{Chapter 3}

\section{Upward swimming of competent}

\section{oyster larvae (Crassostrea virginica) persists in highly turbulent flow as detected by PIV flow subtraction}

\subsection{Abstract}

${ }^{1}$ Investigating settlement responses in the transitory period between planktonic and benthic stages of invertebrates helps shape our understanding of larval dispersal and supply, as well as early adult survival. Turbulence is a physical cue which has been shown to induce sinking and potentially settlement responses in mollusc larvae. In this study, we determine the effect of turbulence on vertical swimming velocity and diving responses in competent eastern oyster larvae (Crassostrea virginica). We quantified the behavioral responses of larvae in a moving flow field by measuring and analyzing larval velocities in an relative framework (where local flow is subtracted away, isolating the behavioral component) in contrast to the more common absolute framework (in which behavior and advection by the flow are conflated). We achieved this separation by simultaneously and separately tracking individuals and measuring the

\footnotetext{
${ }^{1}$ Originally published as "JD Wheeler, KR Helfrich, EJ Anderson, B McGann, P Staats, AE Wargula, K Wilt, and LS Mullineaux (2013). Upward swimming of competent oyster larvae (Crassostrea virginica) persists in highly turbulent flow as detected by PIV flow subtraction. Marine Ecology Progress Series. 488: 171-185." Reproduced here under the terms of the Creative Commons Attribution License, which permits use, distribution and reproduction in any medium, provided the original work is properly cited.
} 
flow field around them using particle image velocimetry (PIV) in a grid-stirred turbulence tank. Contrary to our expectations, larvae swam upward even in highly turbulent flow, and the dive response became less frequent. These observations suggest that oyster larvae are stronger swimmers than previously expected and provide evidence that turbulence alone may not always be a sufficient cue for settlement out of the water column. Furthermore, at a population level, absolute velocity distributions differed significantly from isolated larval swimming velocities, a result that held over increasing turbulence levels. The absolute velocity distributions indicated a strong downward swimming or sinking response at high turbulence levels, but this observation was in fact due to downwelling mean flows in the tank within the imaging area. Our results suggest that reliable characterization of larval behavior in turbulent conditions requires the subtraction of local flow at an individual level, imposing the technical constraint of simultaneous flow and behavioral observations.

\subsection{Introduction}

Planktonic larval organisms live in a turbulent ocean environment, and their interactions with this complex physical system have profound impacts on life beyond the small scales at which the interactions occur. Studying the environmental cues that trigger settlement responses in the transitory period between planktonic and benthic stages of invertebrates may be important for our understanding of larval dispersal and supply, as well as early adult survival. Behavioral changes in larvae in response to environmental cues may occur in the benthic boundary layer, allowing them to differentiate between micro-habitats on the centimeter scale (e.g. [91, 92]), or higher in the water column. In the water column, behavioral responses may be regulated by temperature, salinity, and light gradients, which operate on larger spatial scales than the localized responses in the bottom boundary layer (for review, see [12]). One such behavioral response is vertical swimming, an important consideration when vertical larval swimming speeds can overcome vertical flow. Self-governed vertical position in the water column affects simulated large-scale dispersal patterns (e.g. [14, 15]) except when vertical mixing is strong [16]. Vertical position can be especially important for larvae accumulating in vertically sheared flows or fronts, as such flows may facilitate larval transport to nearshore settlement environments (e.g. [17, 18, 19, 20]), or under different conditions, act as a barrier (e.g. [21, 22]). 
Turbulence is another physical cue which may induce behavioral responses in invertebrate larvae. Given potential strong cross-shore gradients in turbulence, properties of flow may alert larvae as they approach nearshore settlement sites. Downward swimming, sinking, or diving in competent larvae may indicate a behavioral settlement response. In mollusc larvae, such sinking behavior could potentially be pronounced, as their dense shells impart sinking velocities much greater than their maximum sustained swimming velocities (e.g. [93]). Sinking in high turbulence was reported by Fuchs et al. [25, 94] for larval mud snails (Ilyanassa obsoleta). Such responses are not limited to molluscs: Clay \& Grünbaum [46] reported downward swimming in eight-armed sand dollar plutei in strong shear flows.

We chose to study the eastern oyster (Crassostrea virginica), since their larvae have been observed to regulate vertical position in the water column [93, 95] and dive in moderately turbulent channel flows [96]. Turbulence-mediated recruitment is also a current issue for the conservation of wild oyster populations [97]. Our main objectives are to isolate and quantify the behavioral component of larval vertical velocity (relative to the surrounding flow), determine if larval vertical velocity varies with turbulence level, and identify the dive response to determine if it increases in frequency in highly turbulent flow, resulting in a significant change in downward motion of the population as a whole.

The first objective presents a challenging technical problem, in attempting to quantify both larval swimming velocities and local flow velocities simultaneously. Previous experimental studies have investigated invertebrate larval behavior in grid-stirred turbulence tanks $[25,66,52]$, tank shear flow generated by temperature gradients $[46,66]$, and turbulent flume flows $[48,98,99]$. Behavioral swimming metrics primarily focused on absolute metrics (without flow subtraction), such as absolute vertical velocity [52] and absolute swimming direction [66, 52]. In Fuchs et al. [25], larval swimming velocities were approximated by mean flow subtraction: average flow velocities from time series were recorded at multiple points in the tank subsequent to larval trials using laser Doppler velocimetry, and these mean flow values were subsequently subtracted from observed larval swimming velocities. In McDonald [66] and Roy et al. [52], passive particles of comparable size to the larvae were tracked in an identical experimental set-up to the larval trials, in order to determine differences between swimming in flow and passive advection in flow. These methods of assessing population-level behavioral metrics rely on strict assumptions of spatial and temporal homogeneity of flow which are likely unrealistic and may result in inaccurate estimates of flow near individual 
larvae and hence erroneous larval behavioral velocities.

We propose that simultaneous tracking of larvae and the surrounding flow is necessary to determine larval behavior, to fully decouple swimming from advection. This is certainly necessary for identifying individual-level behaviors such as diving, but we further propose that even on a population level, swimming behavior cannot be discerned from absolute velocity values, instead requiring the local flow to be subtracted from each individual larval trajectory.

Only Clay \& Grünbaum [46] have previously endeavored to decouple flow from swimming, in a shear flow for sand dollar larvae, by simultaneously tracking passive algae particles in the tank and interpolating the velocity field from the algal trajectories, though they do not report on algal particle density. Our method of determining the flow field, using particle image velocimetry (PIV), may provide a finer temporal and spatial resolution dataset of the recorded flow field.

Further, few grid-stirred tank studies have generated experimental turbulence with energy dissipation rates consistent with nearshore conditions. Tidal channel and estuarine flows attain dissipation rates ranging from $\varepsilon \sim 10^{-2}-10^{0} \mathrm{~cm}^{2} \mathrm{~s}^{-3}[76]$, while surf zone conditions range from $\varepsilon \sim 10^{-1}-10^{2} \mathrm{~cm}^{2} \mathrm{~s}^{-3}$ [100]. Fuchs et al. [25] conducted turbulence tank studies with maximum $\varepsilon \sim 2.7 \mathrm{~cm}^{2} \mathrm{~s}^{-3}$, but Roy et al. [52] and McDonald [66] set energy dissipation rates more consistent with off-shore continental shelf mixed layers, due to working with newly hatched and therefore pre-competent sea urchin larvae. In order to address questions of turbulence-mediated settlement effects on oyster larvae, higher turbulence levels are required due to their tendency to settle in shallow intertidal and subtidal zones [6]. Although grid-stirred turbulence lacks the strong mean vertical shear experienced by larvae in a bottom boundary layer, it provides a good system for investigating controlled turbulence in isolation from larger-scale velocity gradients.

The conceptual goal of the present study is to further our understanding of larval oyster settlement behavioral responses in a turbulent water column. We hypothesize that the dive response observed by Finelli \& Wethey [96] is a settlement response that will increase with turbulence in a grid-stirred tank, and that populations of larvae will manifest a downward swimming response (relative to flow) in turbulence through this dive response. Further, we hypothesize that there exists a threshold turbulence level (quantified through energy dissipation rate) above which larvae cease to swim and sink, contributing to a downward 
velocity response to turbulence.

\subsection{Methodology}

\subsubsection{Experimental organism}

Crassostrea virginica, or eastern oyster, is a species of mollusc found along the Atlantic coast of North America from the Gulf of Mexico to the Canadian Maritimes, and inhabits subtidal and intertidal zones in its adult life stage. Spawning occurs in the spring and summer, with a pelagic larval duration of two to three weeks depending on environmental conditions $[101,6]$, although the shell and propulsive velum develop within the first two days [102]. Oyster larvae are competent to settle upon development of eyes and a foot in the pediveliger stage, having shell width exceeding 150-200 $\mu \mathrm{m}$ [103].

\subsubsection{Culturing of larvae}

Larvae were obtained when near-competent (retained on a $180 \mu \mathrm{m}$ mesh) from the Aquaculture Research Corporation (ARC), a shellfish hatchery in Dennis, MA. Larvae were maintained in aerated $0.3 \mu \mathrm{m}$-filtered seawater (16 L containers) having ambient field surface temperature $\left(20-22^{\circ} \mathrm{C}\right.$ ) and salinity (approximately $33 \mathrm{psu}$ ), at densities of 3000 larvae per litre. Larvae were fed once daily a $375 \mathrm{~mL}$ solution of haptophyte Isochrysis sp. in filtered seawater, having algal density of $9 \times 105$ cells $\mathrm{mL}^{-1}$. Once obtained from the hatchery, larvae were used in turbulence experiments within two days, to prevent settlement to the bottom of culture buckets. In preliminary experiments, larval shell height at the time of use averaged $220 \mu \mathrm{m}$. Larval shell height was not measured for this study, but randomly sampled larvae were examined to ensure the presence of the eyespot and well-developed foot, indicating competency to settle.

\subsubsection{Experimental tank}

A grid-stirred plexiglass tank $(44.5 \times 44.5 \times 90 \mathrm{~cm})$ was placed in an environmental chamber of fixed temperature $\left(20^{\circ} \mathrm{C}\right)$ and filled with $0.3 \mu \mathrm{m}$-filtered seawater of ambient field surface temperature (approximately $20^{\circ} \mathrm{C}$ ). Two grids, constructed from $1 \times 1 \mathrm{~cm}$ plexiglass bars spaced $5 \mathrm{~cm}$ apart, were connected equidistantly above and below the experimental field of 
view, and covered the horizontal cross section of the tank (Fig. 3-1). These grids were vertically connected by narrow steel bars and attached to a motor above the tank which oscillated the grids vertically with amplitude $5 \mathrm{~cm}$ at a specified frequency. A high speed monochrome camera (Photron Fastcam SA3) and a pulsed near-infrared laser (Oxford Lasers, Firefly $300 \mathrm{~W}, 1000 \mathrm{~Hz}, 808 \mathrm{~nm}$ ) were trained perpendicularly to illuminate and record a $2.5 \times 2.5$ $\mathrm{cm}$ field of view (FOV) in the centre of the tank, with a laser sheet thickness of approximately $1 \mathrm{~mm}$. A near-IR laser was used because preliminary experiments with a green laser $(532 \mathrm{~nm})$ showed that larvae reacted strongly (dove downward) when exposed to flashes of visible light. The environmental chamber was therefore kept in darkness for the duration of the experiment.

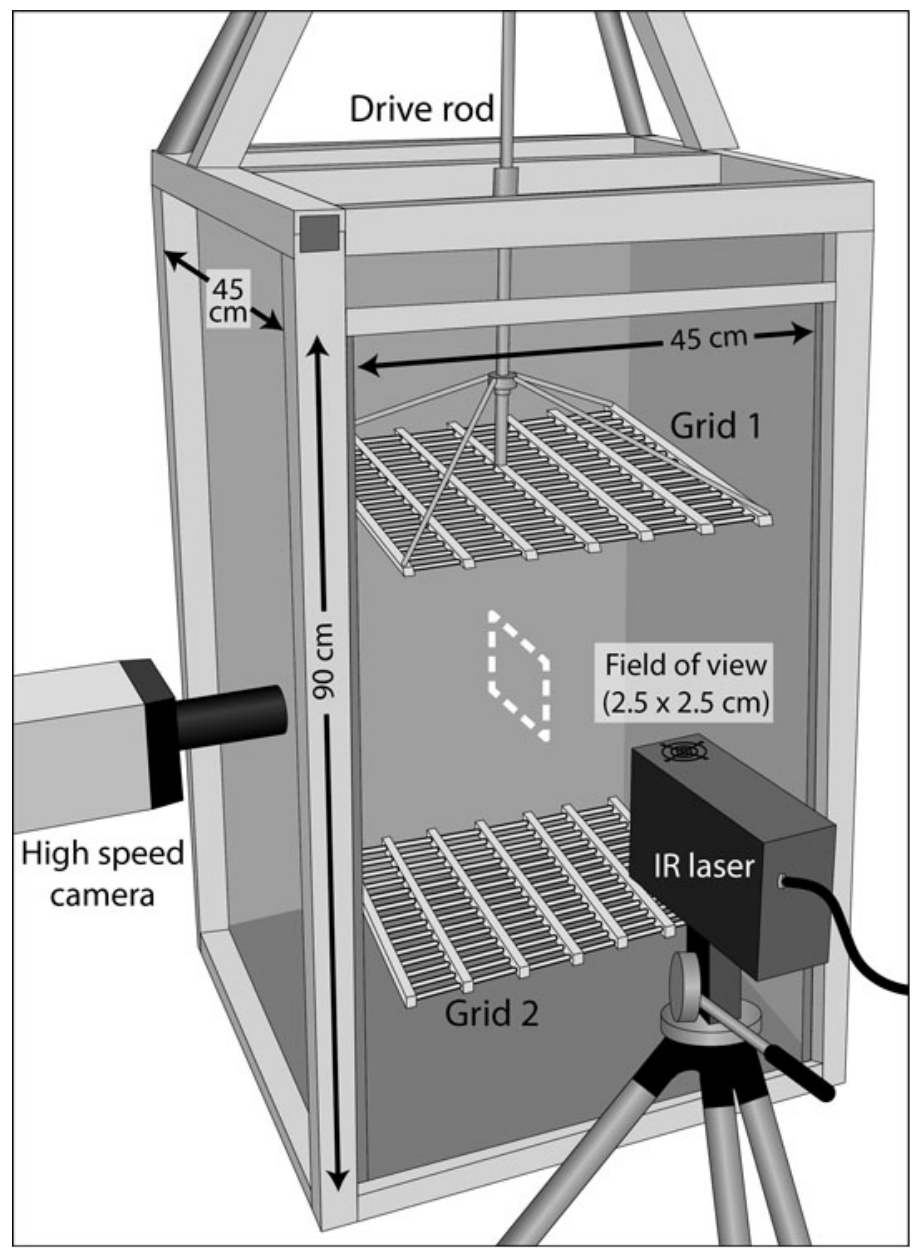

Figure 3-1: Experimental tank set-up, with two vertically oscillating grids to generate flow and the perpendicularly trained camera and laser to image a $2.5 \times 2.5 \mathrm{~cm}$ field of view in the center of the tank. 


\subsubsection{Experimental Procedure}

Separate batches of larvae were introduced into the tank as replicates; each batch is identified by a different trial number (T1 through T4). Within each trial, multiple 45 second videos (datasets) were recorded for each turbulence level (or treatment). At the end of each trial, the tank was drained and rinsed, with the larvae discarded. Two separate two-day experiments (denoted OY5 and OY6) were carried out on different dates, using separate cohorts of larvae from ARC.

Larvae were poured into the tank from a beaker, with numbers ranging from 85,000 105,000 larvae (0.5-0.62 larvae $\left.\mathrm{mL}^{-1}\right)$ for each trial. A total volume of $2.5 \mathrm{~cm}^{3}$ of an aqueous solution of neutrally-buoyant polystyrene particles of diameter 3-3.4 $\mu \mathrm{m}$ (Spherotech Inc., $5 \%$ weight by volume) were injected into the tank to act as passive particles for PIV analysis. Polystyrene particles, rather than natural algae, were necessary to get sufficient light scattering for PIV. Although feeding experiments showed that larvae did ingest particles on time scales associated with a single trial (3 hours), the ingestion had no significant effect on larval fall velocities. Once particles and larvae were added to the tank, the tank was covered and left undisturbed for twenty minutes prior to the initialization of a trial, to allow dissipation of flows generated from tank filling and larvae addition. We saw no evidence of the particle-induced behaviors reported by Fuchs et al. [27], who used $12 \mu \mathrm{m}$ glass and 20 $\mu \mathrm{m}$ nylon particles, considerably larger than our $3 \mu \mathrm{m}$ polystyrene particles.

In each trial, treatment order was randomly determined after an initial still water treatment. Still water treatments were conducted at the beginning of each trial due to the difficulties in spinning down the tank completely after higher turbulence treatments. Treatments of increasing turbulence were conducted by oscillating the vertical grid structure with frequencies $0,0.25,0.5,0.75,1.25$, and $1.75 \mathrm{~Hz}$ and fixed amplitude $5 \mathrm{~cm}$. These turbulence levels will hereafter be referred to as levels $\ell_{0}, \ell_{0.25}, \ell_{0.5}, \ell_{0.75}, \ell_{1.25}$, and $\ell_{1.75}$. In each treatment, datasets were recorded after an initial spin-up time of five minutes to the appropriate turbulence level. Datasets corresponded to approximately 45 seconds of footage at $60 \mathrm{fps}$. Approximately 5 minutes elapsed between the recording of each dataset, due to data transfer times between the camera and hard drive. In experiment OY5, 4 datasets were recorded for each treatment, up to $\ell_{1.25}$. In OY6, the number of datasets was adjusted to improve the number of larval tracks, based on observations from OY5. The number of 


\begin{tabular}{|l|l|l|l|l|}
\hline Turb. level & $\begin{array}{l}\text { Grid } \\
\text { Freq. }(\mathrm{Hz})\end{array}$ & $\#$ Datasets & Time step $\left(\mathrm{s}^{-1}\right)$ & $\begin{array}{l}\text { Dissip. rate } \varepsilon \\
\left(\mathrm{cm}^{2} \mathrm{~s}^{-3}\right)\end{array}$ \\
\hline$\ell_{0}$ & 0 & $4(\mathrm{OY} 5), 5(\mathrm{OY} 6)$ & 0.0167 & Approaches 0 \\
\hline$\ell_{0.25}$ & 0.25 & $4(\mathrm{OY} 5), 5(\mathrm{OY} 6)$ & 0.0167 & 0.002 \\
\hline$\ell_{0.5}$ & 0.5 & $4(\mathrm{OY} 5), 5(\mathrm{OY} 6)$ & 0.0167 & 0.017 \\
\hline$\ell_{0.75}$ & 0.75 & $4(\mathrm{OY} 5), 4(\mathrm{OY} 6)$ & 0.0167 & 0.064 \\
\hline$\ell_{1.25}$ & 1.25 & $4(\mathrm{OY} 5), 3(\mathrm{OY} 6)$ & $0.0075(\mathrm{OY} 5), 0.008(\mathrm{OY} 6)$ & 0.373 \\
\hline$\ell_{1.75}$ & 1.75 & $0(\mathrm{OY} 5), 4(\mathrm{OY} 6)$ & 0.006 & 0.667 \\
\hline
\end{tabular}

Table 3-1: Detail of experimental setup for each turbulence level. Grid frequency is the rate of vertical oscillation of double grids. Datasets are the replicate trials in experiments OY5 and OY6. Time step is the time delay between two paired PIV images. Dissipation rate $\varepsilon$ calculated as in Doron et al. [104].

datasets in OY6 was 5, 5, 5, 4, 3 and 4, respectively for turbulence levels from $\ell_{0}$ to $\ell_{1.75}$. The $\ell_{1.75}$ turbulence level was added in OY6.

For all treatments, the camera recorded images at $60 \mathrm{fps}$. For treatments up to turbulence level $\ell_{0.75}$, the laser flashed at $60 \mathrm{~Hz}$, while at higher turbulence $\left(\ell_{1.25}\right.$ and $\left.\ell_{1.75}\right)$, a frame straddling method was employed between the camera and the laser, where the camera triggered the laser to delay then pulse for brief intervals during the recording, effectively creating successive pairs of images having long and short time steps. All high-turbulence analysis was conducted on the short time step image pairs, to facilitate correlation of passive particle position during subsequent PIV analysis. These time steps were $0.0075 \mathrm{~s}^{-1}$, $0.008 \mathrm{~s}^{-1}$, and $0.006 \mathrm{~s}^{-1}$ for OY5 $\ell_{1.25}$, OY6 $\ell_{1.25}$, and OY $6 \ell_{1.75}$, respectively. Relevant experimental values (grid frequencies, numbers of datasets, and time steps) are summarized in Table 3-1. Experimental images from each dataset were saved as high-resolution (1024× 1024 pixel) TIFF images and subjected to analysis for larval tracking and flow visualization.

\subsubsection{Data analysis}

Individual larval velocity in flow is composed from the sum of both larval swimming and advection by the flow field. We denote individual vertical larval swimming velocity as $w_{s}$, and vertical local flow velocity as $w_{f}$, so that the absolute vertical larval velocity $w_{a}$ observed for an individual is

$$
w_{a}=w_{s}+w_{f}
$$

In the absence of a well-resolved flow field in space, as would occur in the absence of PIV data, one could approximate $w_{f}$ with a temporal average $\left\langle w_{f}\right\rangle$ at a point or set of points in 
space. We denote larval vertical swimming velocities calculated using this approximation as mean-shifted swimming velocities, or $w_{m s}$ :

$$
w_{m s}=w_{a}-\left\langle w_{f}\right\rangle
$$

We will assess the validity of this spatial and temporal flow field averaging when isolating larval swimming velocities, to determine if simultaneous quantification of flow and larval swimming are necessary. The methods by which these values are determined experimentally are addressed in the following two subsections. The latter two subsections address the identification of the dive response in larval trajectories and the method by which the energy dissipation rate is calculated for a given turbulence level.

\section{Larval tracking}

Absolute vertical larval velocities $w_{a}$ were obtained using the following larval tracking procedure. All TIFF images were filtered to remove average background intensity and passive particles (prior to filtering, images were predominantly black with larvae appearing as white spots and smaller passive particles appearing as a pale haze) using LabVIEW 2010 (National Instruments) software. Centroid positions ( $x$ and $z$ coordinates) of larvae were identified using the software, along with body size and frame number in which they were identified. An in-house MATLAB (v.7.12.0 R2011a) code was developed to track each individual larva from frame to frame, according to a user-specified tolerance radius in which the larva could be found in a subsequent frame. Larval tracks were identified using this method. All tracks of fewer than five frames in length were discarded (usually due to the larva passing out of the FOV too quickly). Instantaneous velocities were then calculated from larval positions in each pair of frames to obtain individual absolute vertical larval velocities $w_{a}$.

\section{Quantification of flow field and isolation of larval swimming}

Local flow fields were quantified using the following PIV methodology to calculate vertical flow velocities $w_{f}$ local to each larva. Experimental TIFF files were first imported into LaVision DaVis (v.7.2) imaging software. Each experimental image or frame showed the entire FOV for a given time step. For $\ell_{1.25}$ and $\ell_{1.75}$, the frames were grouped $1+2,3+4$, etc. prior to analysis due to frame straddling in the camera/laser set-up. Each frame was 
subdivided into final interrogation windows of $16 \times 16$ pixels (multi-pass interrogation with no overlap), and cross-correlations were computed between the particles in the interrogation window and in shifting search windows in the next frame, to determine the most probable location of the same particles in the next frame. We used the DaVis default FFT with Whittaker reconstruction as the correlation function. For a given dataset, the PIV analysis generated two $64 \times 64$ matrices for each time step, corresponding to the horizontal and vertical flow velocities on a grid in the FOV (Fig. 3-2), with a spatial resolution of 0.039 $\mathrm{cm}$ between grid points.

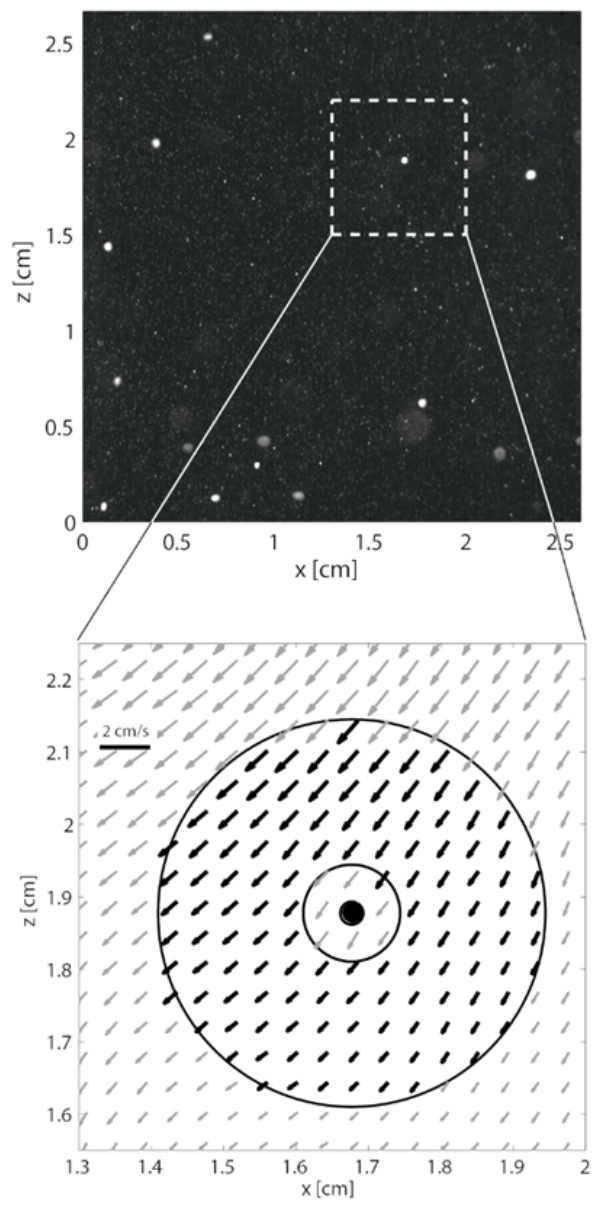

Figure 3-2: Image of field of view in turbulence tank, where larvae are bright white spots and passive particles are smaller specks. Large diffuse spots are out-of-focus larvae (filtered out prior to analysis). The inset image is a sample velocity field determined from PIV analysis, where the larval position is the solid black circle, and all black velocity vectors fall within the annulus by which local flow velocity $w_{f}$ was calculated for the given larva. 
These velocity fields were converted into MATLAB data files, and were integrated into the larval tracking code. Each larval position identified a dynamic annulus with an inner radius corresponding to the sum of two values: the maximum larval radius in the dataset and the PIV spatial grid interval. The outer radius was 4 times greater than the inner radius (Fig. 3-2). Such an annulus was selected to account for blurring and poor PIV results in the larval boundary layer. Further, the flow very close to the larva would be subject to larval propulsion and wake, and we wished to avoid this bias to the local flow. Results were not sensitive to the outer radius of the annulus, as the flow remained coherent for six to eight PIV velocity vectors away from the larva. Within the annulus for each larva in each frame, the mean vertical velocity from the PIV analysis was computed, and this mean velocity was identified as the local vertical flow velocity around the larva, $w_{f}$. For each larva in each frame, larval swimming velocities $w_{s}$ were obtained from $w_{s}=w_{a}-w_{f}$.

The flow data from the PIV analysis was also used to determine the average flow velocity in each dataset. The center points of each quadrant in the FOV were selected and the flow velocity time series of each dataset were extracted from the PIV data (Fig. 3-3). The average flow velocity in the FOV was calculated from these four time series for each dataset to give $\left\langle w_{f}\right\rangle$, and the mean-shifted larval swimming velocities were obtained from $w_{m s}=w_{a}-\left\langle w_{f}\right\rangle$.

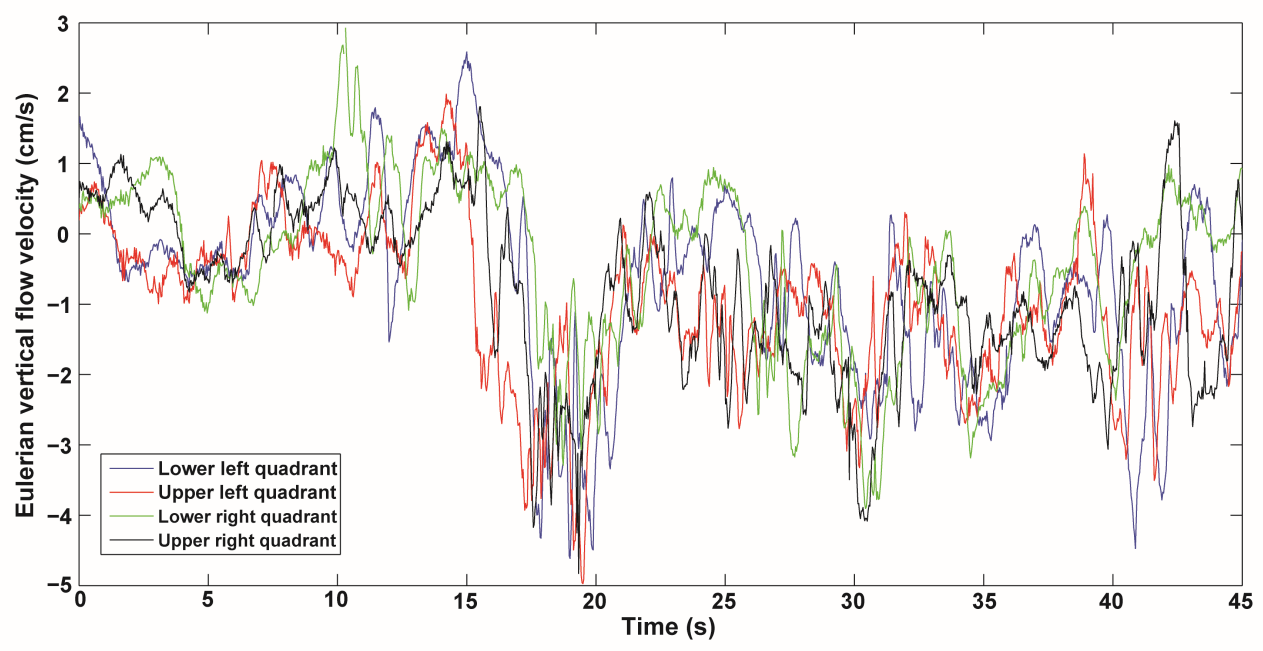

Figure 3-3: Vertical velocity time series at central points in the four quadrants of the field of view, selected from a level $\ell_{1.25}$ dataset in OY 5 , used to calculate $\left\langle w_{f}\right\rangle$. 


\section{Identification of dive response}

The dive response was identified in larval tracks through extensive comparison of experimental footage and corresponding individual swimming velocity time series. Through visual identification of the dive response in the experimental footage, we found a corresponding signature in the time series data, comprising of a sudden (within 1-2 time steps) drop in vertical velocity to swimming velocities in the range of -0.4 to $-0.8 \mathrm{~cm} \mathrm{~s}^{-1}$. The larval descent would then slow over 10-20 time steps before the vertical velocity would stabilize. Larval trajectories were classified as dive response tracks if they reached accelerations of 3.0 $\mathrm{cm} \mathrm{s}^{-2}$ (approximately 150 body lengths $\mathrm{s}^{-2}$ ) for more than one time step, and achieved negative vertical velocities of at least $-0.4 \mathrm{~cm} \mathrm{~s}^{-1}$.

\section{Calculation of turbulent energy dissipation rate}

Energy dissipation was estimated through a separate set of PIV-only experiments in the same grid-stirred turbulence tank. These tests employed a green Nd/Yag laser (Big Sky) and a LaVision Imager Pro X camera $(2048 \times 2048$ pixels $)$. Sphericel@ $10 \mu \mathrm{m}$ hollow glass spheres were used as the tracer particles in $3 \mu \mathrm{m}$-filtered seawater. The image FOV was $12 \times 12 \mathrm{~cm}$, centered in the tank. At each turbulence level, 3 sequences of 150 image pairs were acquired at $4 \mathrm{~Hz}$ for later PIV analysis using LaVision DaVis PIV software. The interframe time was adjusted between turbulence levels for optimal PIV analysis. Velocity vectors were computed using $32 \times 32$ pixels interrogation regions with 50 percent overlap resulting in $128 \times 128$ vectors with separation of $0.119 \mathrm{~cm}$. Dissipation rates $\varepsilon$ were calculated directly from the two-dimensional vector fields from Doron et al. (2001):

$$
\varepsilon=3 \nu\left[\overline{\left(\frac{\partial u}{\partial x}\right)^{2}}+\overline{\left(\frac{\partial u}{\partial z}\right)^{2}}+\overline{\left(\frac{\partial w}{\partial x}\right)^{2}}+\overline{\left(\frac{\partial w}{\partial z}\right)^{2}}+\overline{2\left(\frac{\partial u}{\partial z} \frac{\partial w}{\partial x}\right)}+\frac{2}{3} \overline{\left(\frac{\partial u}{\partial x} \frac{\partial w}{\partial z}\right)}\right]
$$

Here $u$ and $w$ are velocities in the horizontal, $x$, and vertical, $z$, directions, respectively, and $\nu$ is the kinematic viscosity of seawater. The overbar indicates an average over the FOV and time at a fixed turbulence level. Prior to computing, $\varepsilon$ the individual vector fields were passed through a median filter and the derivatives were then estimated using leastsquares finite difference stencils to reduce noise (see [105]). Since the flow is inherently threedimensional, the estimate of dissipation rate from two-dimensional velocity fields assumes that the turbulence is isotropic and that the out of plane, $y$-direction, velocity fluctuations, 
$v$, have average magnitudes equal to the in-plane values (see [104]). These criteria are not precisely met in a grid-driven flow. However, the symmetry of the grid implies that $u \sim v$. Furthermore, the measurements show that the ratio of $w_{r m s}$ to $u_{r m s}$ of root-mean-squared values is order one, ranging from $\approx 2-1$ from the lowest to highest turbulence levels considered. This anisotropy is typical of grid-forced turbulence. The central location of the FOV between grids promotes homogeneity of the turbulence.

\subsubsection{Statistical analysis}

The primary objective of this paper was to determine differences between larval vertical swimming velocities in varying levels of turbulent flow. However, prior to this analysis, differences between absolute and larval swimming velocity distributions in each turbulence level were determined, to examine robustness of previous methodologies in decoupling larval behavior from flow. Distributions of larval velocities (denoted by $W$ and an appropriate subscript) are constructed by combining all individual instantaneous velocities, so that $W_{s}$, for instance, is the distribution of larval swimming velocities $w_{s}$, for a given turbulence level. Statistical analyses were carried out to compare larval swimming velocity distributions $W_{s}$, calculated by our PIV methods to the more typically used mean-shifted swimming velocity distributions $W_{m s}$. We also determined statistical differences between larval swimming distributions $W_{s}$ over increasing turbulence levels. Finally, we determined the statistical significance of changes in dive frequency with respect to turbulence level. Because of the non-independence of the data in the distributions (velocity of a larva at time step $t$ is likely correlated to velocity in nearby time steps), all statistical analyses were conducted on the mean instantaneous velocity of each larval track. All statistical analyses, unless otherwise referenced, were carried out using MATLAB statistical routines at a significance level of $\alpha=0.05$ with the appropriate Bonferroni correction.

For each trial in each turbulence level, we compared the means and the variances of the mean-shifted swimming velocity distribution $\left(W_{m s}\right)$ and isolated swimming velocity distribution $\left(W_{s}\right)$, using a modified two-tailed $t$ test (Welch's approximate $t$ test statistic and Satterthwaite's approximation for the degrees of freedom) for means and the two-tailed variance ratio test for the variances.

To determine the effect of turbulence level on the mean vertical swimming velocity, and also the effect of turbulence treatment order, we conducted a two-way ANOVA. Significance 
levels in ANOVAs can be adversely affected by the combined effects of heterogeneity of variance and sample size, but the effect of non-homoscedasticity on the ANOVA's significance level are almost negligible when sample sizes are equal [106]. We therefore sub-sampled the distributions in each turbulence level and trial so the sample size in each matched the smallest sample in the initial set. Though the larval population in the tank remained the same within a trial for the different turbulence levels, the experimental design does not call for a repeated-measures analysis, as the large quantity of larvae in the tank and frequent mixing suggests that resampling of larvae in the FOV was rare. Two subsequent tests were implemented in the case of a significant ANOVA result. First, multiple comparisons of the mean swimming velocities in the different turbulence levels were carried out using Tukey-Kramer's honestly significant difference criterion, and second, one-tailed $t$ tests were implemented to determine if vertical directional swimming was significantly different from zero for each turbulence level. These two sets of tests are complementary, as the $t$ test compares larval swimming to a zero velocity baseline while the multiple comparison test examines the differences in swimming velocity between turbulence levels.

Dive frequencies were compared between turbulence levels using a $\chi^{2}$ binomial comparison test with Yates' corrected $\chi^{2}$ test statistic [107], to determine if the proportion of larvae which dove was dependent on the turbulence level. The contingency tables of the binomial comparison test were then sub-divided to identify the turbulence threshold at which a significant difference in dive frequency was detected.

\subsection{Results}

\subsubsection{Energy dissipation rate}

Using a kinematic viscosity of $\nu=0.01 \mathrm{~cm}^{2} \mathrm{~s}^{-1}$ for seawater, we found the following energy dissipation rates $\varepsilon$ for the experimental turbulence levels: $\varepsilon_{\ell 0} \rightarrow 0, \varepsilon_{\ell 0.25}=0.002, \varepsilon_{\ell 0.5}=$ 0.017, $\varepsilon_{\ell 0.75}=0.064, \varepsilon_{\ell 1.25}=0.373, \varepsilon_{\ell 1.75}=0.667 \mathrm{~cm}^{2} \mathrm{~s}^{-3}$ (where $\ell$ denotes the grid oscillation frequency, Table 3-1). This range of dissipation rates is consistent with those recorded in tidal channels, estuaries, and calmer surf zones [76, 100], and therefore suitable for testing oyster larvae settlement responses. The Kolmogorov length scale at the highest experimental turbulence level $(0.035 \mathrm{~cm})$ is comparable to the spatial resolution of the PIV data $(0.039 \mathrm{~cm})$, and so we capture the smallest-scale flow dynamics in the turbulence tank. 

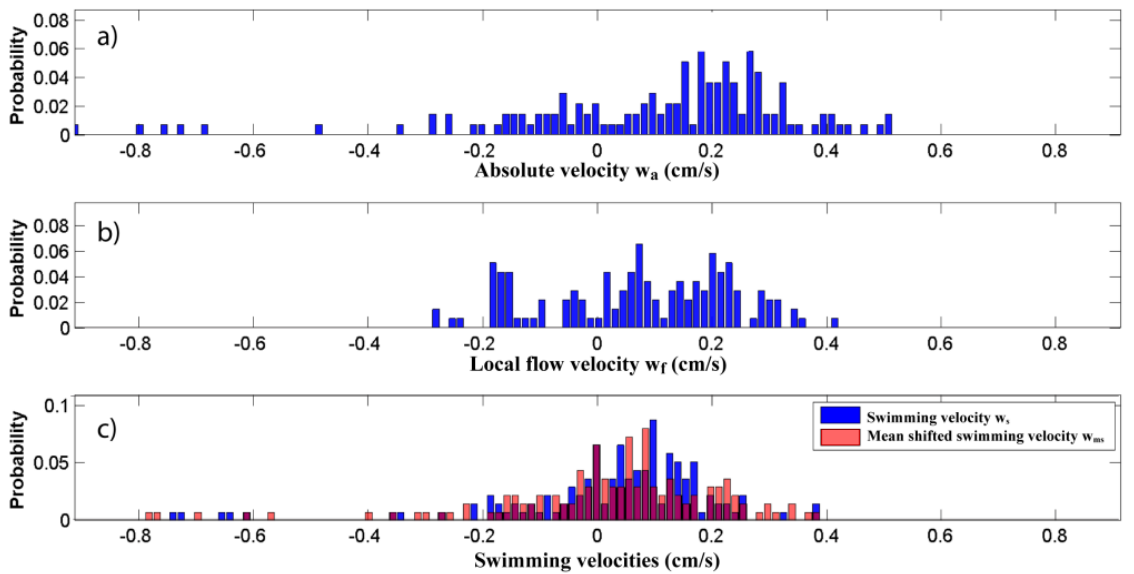

OY6 10.75
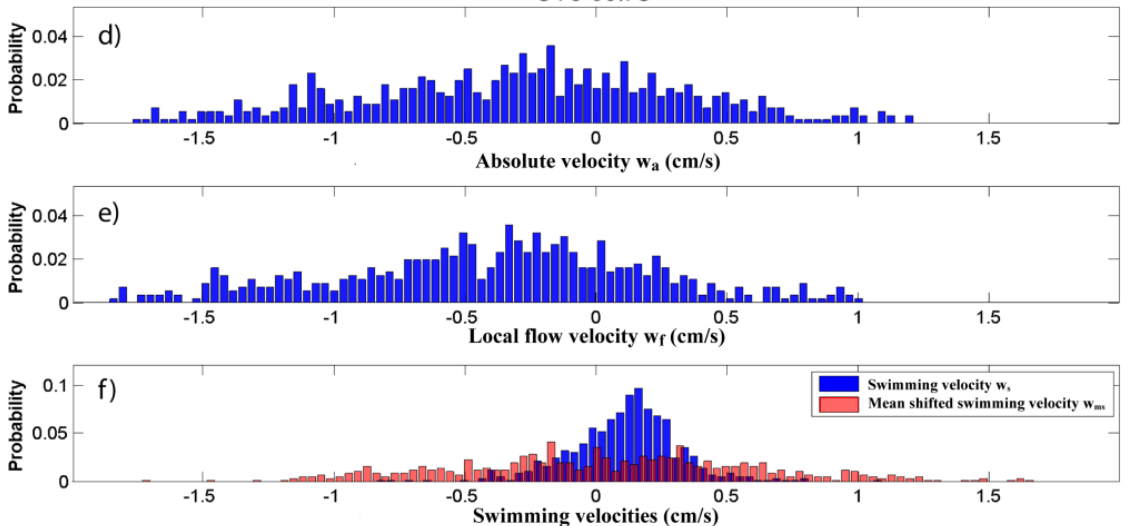

OY6 $\ell 1.75$
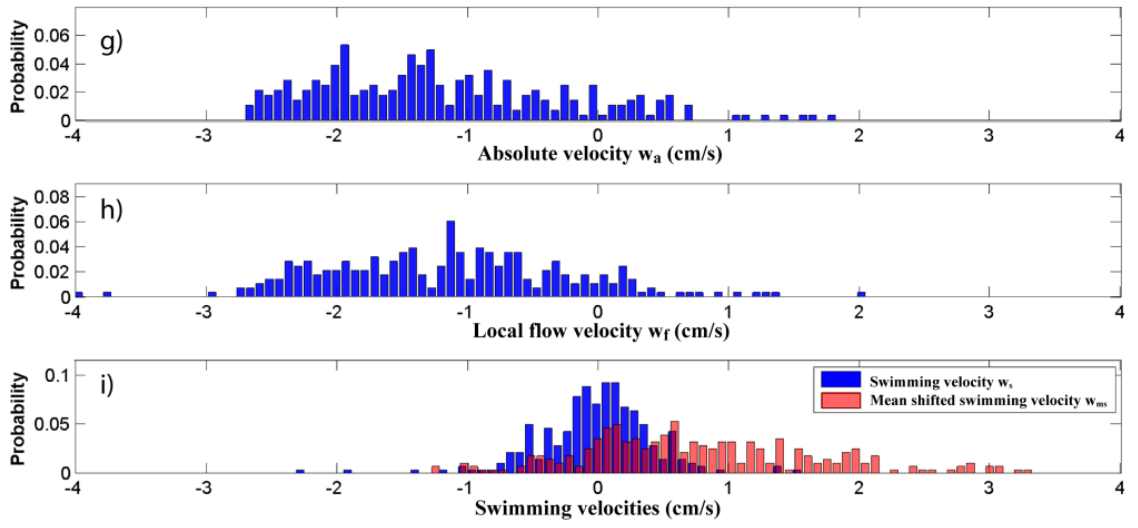

Figure 3-4: A comparison of vertical instantaneous larval and flow velocity distributions for turbulence levels $\ell_{0}, \ell_{0.75}$, and $\ell_{1.75}$. For each level, the top panel illustrates mean absolute velocities ( $\mathrm{a}, \mathrm{d}, \mathrm{g}$ ), the mid panel illustrates mean flow velocities in an annulus around each larva $(\mathrm{b}, \mathrm{e}, \mathrm{h})$. The bottom panel shows mean swimming velocity, computed by subtracting the local flow around each larva from the absolute velocity, in blue, and mean-shifted swimming velocity, in red (c, f, i). Turbulence levels have separate horizontal axis scaling for clarity. 


\subsubsection{Absolute versus isolated vertical swimming velocity}

In all turbulence levels except $\ell_{0}$ and $\ell_{0.25}$, the variances of the mean-shifted swimming velocity distributions and swimming velocity distributions ( $W_{m s}$ vs $\left.W_{s}\right)$ differed significantly

in all trials. In $\ell_{0}$, where no flow was induced in the tank, the variances in $W_{m s}$ and $W_{s}$ were not significantly different in five of the eight trials and in $\ell_{0.25}$, the variances were indistinguishable in one of eight trials (Table 3-2). At all other turbulence levels, the variances of these two distributions were significantly different in all trials ( 8 of 8 in $\ell_{0.50}-\ell_{1.25}$ and 4 of 4 in $\left.\ell_{1.75}\right)$. Clearly, the variance of the larval swimming velocity distribution cannot be captured by the mean-shifted velocity distribution when even weak flow is induced in the tank.

The means of the two distributions were also compared for each trial, in each turbulence level. The means of the two distributions were statistically different in no trials in $\ell_{0}, 2$ of 8 trials in $\ell_{0.25}$ and $\ell_{0.5}, 5$ of 8 trials in $\ell_{0.75}, 1$ of 8 trials in $\ell_{1.25}$, and 4 of 4 trials in $\ell_{1.75}$ (Table $3-3)$. It appears that $W_{m s}$ approximates the mean swimming velocity well when no flow is induced in the tank, but the approximation is not as reliable when flow is induced. At high turbulence, the mean-shifted approximation appears good in $\ell_{1.25}$ but fails to capture the swimming velocity distribution in $\ell_{1.75}$.

An example of larval velocity distributions in increasing turbulence levels (Figure 3-4) demonstrates this disagreement between the two approaches. From top to bottom, each set of three panels represents velocity distributions in $\ell_{0}, \ell_{0.75}$, and $\ell_{1.75}$, respectively. For each turbulence level, panels illustrate distributions of absolute larval velocity $\left(W_{a}\right)$, larval swimming velocity $\left(W_{s}\right)$, and mean-shifted larval swimming velocity $\left(W_{m s}\right)$, along with the velocity distribution of mean local flow in the annulus around each larva $\left(W_{f}\right)$. The results of the statistical analysis can be readily seen in comparisons of larval swimming velocity and mean-shifted swimming velocity: for still water $\left(\ell_{0}\right)$, the mean and variance of the two distributions are approximately equal (Figure 3-4 c), while for higher turbulence levels $\left(\ell_{0.75}\right.$ and $\ell_{1.75}$ ), the variance in $W_{m s}$ distribution is significantly larger than that of $W_{s}$ (Figs. 3-4 $\mathrm{f}$ and i). The means of these distributions in $\ell_{0}$ and $\ell_{0.75}$ appear roughly equal, but at $\ell_{1.75}$, $W_{m s}$ is centered far to the right of $W_{s}$. 


\begin{tabular}{|c|c|c|c|c|}
\hline Trial & $d f$ & $f$ stat & $\begin{array}{l}95 \% \text { CI on } \\
\sigma_{s a}^{2} / \sigma_{r}^{2}\end{array}$ & p-value \\
\hline $\begin{array}{l}\text { OY5 } \ell_{0} \mathrm{~T} 1 \\
\text { T2 } \\
\text { T3 } \\
\text { T4 }\end{array}$ & $\begin{array}{l}54 \\
223 \\
137 \\
125\end{array}$ & $\begin{array}{l}1.76 \\
1.18 \\
1.15 \\
1.15\end{array}$ & $\begin{array}{l}{[1.00,2.96]} \\
{[0.91,1.53]} \\
{[0.82,1.61]} \\
{[0.82,1.63]}\end{array}$ & $\begin{array}{l}0.04 \\
0.21 \\
0.4 \\
0.43\end{array}$ \\
\hline $\begin{array}{l}\text { OY5 } \ell_{0.25} \mathrm{~T} 1 \\
\text { T2 } \\
\text { T3 } \\
\text { T4 }\end{array}$ & $\begin{array}{l}112 \\
203 \\
212 \\
179\end{array}$ & $\begin{array}{l}1.29 \\
1.73 \\
2.17 \\
1.94\end{array}$ & $\begin{array}{l}{[0.89,1.88]} \\
{[1.31,2.29]} \\
{[1.66,2.85]} \\
{[1.44,2.60]}\end{array}$ & $\begin{array}{l}0.16 \\
<\mathbf{0 . 0 0 1} \\
<\mathbf{0 . 0 0 1} \\
<\mathbf{0 . 0 0 1}\end{array}$ \\
\hline $\begin{array}{l}\text { OY5 } \ell_{0.5} \mathrm{~T} 1 \\
\text { T2 } \\
\text { T3 } \\
\text { T4 }\end{array}$ & $\begin{array}{l}100 \\
417 \\
330 \\
260\end{array}$ & $\begin{array}{l}9.57 \\
7.47 \\
6.31 \\
6.99\end{array}$ & $\begin{array}{l}{[6.45,14.20]} \\
{[6.16,9.05]} \\
{[5.08,7.82]} \\
{[5.48,8.92]}\end{array}$ & $\begin{array}{l}<0.001 \\
<0.001 \\
<0.001 \\
<0.001\end{array}$ \\
\hline $\begin{array}{l}\text { OY5 } \ell_{0.75} \mathrm{~T} 1 \\
\text { T2 } \\
\text { T3 } \\
\text { T4 }\end{array}$ & $\begin{array}{l}532 \\
705 \\
587 \\
527\end{array}$ & $\begin{array}{l}4.73 \\
6.44 \\
6.24 \\
6.23\end{array}$ & $\begin{array}{l}{[3.99,5.61]} \\
{[5.55,7.46]} \\
{[5.31,7.34]} \\
{[5.25,7.39]}\end{array}$ & $\begin{array}{l}<0.001 \\
<0.001 \\
<0.001 \\
<0.001\end{array}$ \\
\hline $\begin{array}{l}\text { OY5 } \ell_{1.25} \mathrm{~T} 1 \\
\text { T2 } \\
\text { T3 } \\
\text { T4 }\end{array}$ & $\begin{array}{l}390 \\
591 \\
375 \\
528\end{array}$ & $\begin{array}{l}7.61 \\
8.24 \\
10.51 \\
11.22\end{array}$ & $\begin{array}{l}{[6.23,9.28]} \\
{[7.01,9.68]} \\
{[8.58,12.87]} \\
{[9.46,13.31]}\end{array}$ & $\begin{array}{l}<0.001 \\
<0.001 \\
<0.001 \\
<0.001\end{array}$ \\
\hline $\begin{array}{l}\text { OY6 } \ell_{0} \mathrm{~T} 1 \\
\text { T2 } \\
\text { T3 } \\
\text { T4 }\end{array}$ & $\begin{array}{l}137 \\
366 \\
195 \\
167\end{array}$ & $\begin{array}{l}1.18 \\
1.43 \\
1.59 \\
1.94\end{array}$ & $\begin{array}{l}{[0.84,1.65]} \\
{[1.16,1.75]} \\
{[1.20,2.11]} \\
{[1.43,2.63]}\end{array}$ & $\begin{array}{l}0.33 \\
<0.001 \\
0.001 \\
<0.001\end{array}$ \\
\hline $\begin{array}{l}\text { OY6 } \ell_{0.25} \mathrm{~T} 1 \\
\text { T2 } \\
\text { T3 } \\
\text { T4 }\end{array}$ & $\begin{array}{l}159 \\
122 \\
172 \\
215\end{array}$ & $\begin{array}{l}1.86 \\
3.07 \\
2.76 \\
3.35\end{array}$ & $\begin{array}{l}1.36,2.54] \\
{[2.15,4.39]} \\
{[2.05,3.73]} \\
{[2.57,4.39]}\end{array}$ & $\begin{array}{l}<0.001 \\
<0.001 \\
<0.001 \\
<0.001\end{array}$ \\
\hline $\begin{array}{ll}\text { OY6 } \ell_{0.5} & \text { T1 } \\
\text { T2 } & \\
\text { T3 } & \\
\text { T4 } & \end{array}$ & $\begin{array}{l}453 \\
465 \\
282 \\
241\end{array}$ & $\begin{array}{l}8.05 \\
7.35 \\
7.68 \\
6.48\end{array}$ & $\begin{array}{l}6.69,9.68] \\
{[6.12,8.82]} \\
{[6.08,9.71]} \\
{[5.03,8.35]}\end{array}$ & $\begin{array}{l}<0.001 \\
<0.001 \\
<0.001 \\
<0.001\end{array}$ \\
\hline $\begin{array}{l}\text { OY5 } \ell_{0.75} \mathrm{~T} 1 \\
\text { T2 } \\
\text { T3 } \\
\text { T4 }\end{array}$ & $\begin{array}{l}560 \\
754 \\
380 \\
450\end{array}$ & $\begin{array}{l}7.22 \\
6.15 \\
7.24 \\
6.21\end{array}$ & $\begin{array}{l}6.12,8.52] \\
{[5.33,7.09]} \\
{[5.92,8.86]} \\
{[5.16,7.48]}\end{array}$ & $\begin{array}{l}<0.001 \\
<0.001 \\
<0.001 \\
<0.001\end{array}$ \\
\hline $\begin{array}{l}\text { OY6 } \ell_{1.25} \mathrm{~T} 1 \\
\text { T2 } \\
\text { T3 } \\
\text { T4 }\end{array}$ & $\begin{array}{l}394 \\
626 \\
297 \\
193\end{array}$ & $\begin{array}{l}8.04 \\
15.13 \\
7.3 \\
9.72\end{array}$ & $\begin{array}{l}{[6.23,9.28]} \\
{[7.01,9.68]} \\
{[8.58,12.87]} \\
{[9.46,13.31]}\end{array}$ & $\begin{array}{l}<0.001 \\
<0.001 \\
<0.001 \\
<0.001\end{array}$ \\
\hline $\begin{array}{l}\text { OY6 } \ell_{1.75} \mathrm{~T} 1 \\
\text { T2 } \\
\text { T3 } \\
\text { T4 }\end{array}$ & $\begin{array}{l}281 \\
474 \\
324 \\
91\end{array}$ & $\begin{array}{l}4.17 \\
6.13 \\
5.44 \\
4.96\end{array}$ & $\begin{array}{l}{[3.30,5.27]} \\
{[5.12,7.34]} \\
{[4.37,6.77]} \\
{[3.28,7.51]}\end{array}$ & $\begin{array}{l}<0.001 \\
<0.001 \\
<0.001 \\
<0.001\end{array}$ \\
\hline
\end{tabular}

Table 3-2: Variance ratio test for the mean-shifted swimming velocity $w_{m s}$ versus the swimming velocity $w_{s}$. The null hypothesis states that $\sigma_{\text {shift abs }}^{2}=\sigma_{\text {rel }}^{2}$ while the alternate hypothesis states that these variances are not equal. Significance level is $\alpha=0.05 / 4=0.0125$ (with the Bonferroni correction). 


\begin{tabular}{|c|c|c|c|c|}
\hline Trial & $d f$ & $t$ stat & $\begin{array}{l}95 \% \text { CI on } \\
\left|\mu_{1}-\mu_{2}\right|\end{array}$ & $p$-value \\
\hline $\begin{array}{l}\text { OY5 } \ell_{0} \text { T1 } \\
\text { T2 } \\
\text { T3 } \\
\text { T4 }\end{array}$ & $\begin{array}{l}100.84 \\
442.97 \\
272.62 \\
248.78\end{array}$ & $\begin{array}{l}-1.46 \\
-1.25 \\
-0.81 \\
-0.56\end{array}$ & $\begin{array}{l}{[-.09,0.014]} \\
{[-0.04,0.008]} \\
{[-0.04,0.014]} \\
{[-0.03,0.02]}\end{array}$ & $\begin{array}{l}0.15 \\
0.21 \\
0.42 \\
0.58\end{array}$ \\
\hline $\begin{array}{l}\text { OY5 } \ell_{0.25} \mathrm{~T} 1 \\
\text { T2 } \\
\text { T3 } \\
\text { T4 }\end{array}$ & $\begin{array}{l}220.28 \\
378.45 \\
372.83 \\
324.79\end{array}$ & $\begin{array}{l}-0.45 \\
-0.22 \\
-2.64 \\
-2.19\end{array}$ & $\begin{array}{l}{[-0.06,0.03]} \\
{[-0.04,0.03]} \\
{[-0.06,-0.009]} \\
{[-0.06,-0.003]}\end{array}$ & $\begin{array}{l}0.65 \\
0.82 \\
.008 \\
0.02\end{array}$ \\
\hline $\begin{array}{l}\text { OY5 } \ell_{0.5} \mathrm{~T} 1 \\
\text { T2 } \\
\text { T3 } \\
\text { T4 }\end{array}$ & $\begin{array}{l}120.65 \\
526.62 \\
432.73 \\
332.86\end{array}$ & $\begin{array}{l}0.06 \\
-2.9 \\
-4.7 \\
-1.57\end{array}$ & $\begin{array}{l}{[-0.10,0.11]} \\
{[-0.10,-0.02]} \\
{[-0.15,-0.06]} \\
{[-0.10,0.011]}\end{array}$ & $\begin{array}{l}0.95 \\
.004 \\
<\mathbf{0 . 0 0 1} \\
0.12\end{array}$ \\
\hline $\begin{array}{l}\text { OY5 } \ell_{0.75} \mathrm{~T} 1 \\
\text { T2 } \\
\text { T3 } \\
\text { T4 }\end{array}$ & $\begin{array}{l}747.14 \\
918.72 \\
770.23 \\
691.81\end{array}$ & $\begin{array}{l}-0.009 \\
-0.67 \\
-5.28 \\
-4.63\end{array}$ & $\begin{array}{l}{[-0.04,0.04]} \\
{[-0.06,0.02]} \\
{[-0.18,-0.08]} \\
{[-0.17,-0.07]}\end{array}$ & $\begin{array}{l}0.99 \\
0.5 \\
<\mathbf{0 . 0 0 1} \\
<\mathbf{0 . 0 0 1}\end{array}$ \\
\hline $\begin{array}{l}\text { OY5 } \ell_{1.25} \mathrm{~T} 1 \\
\text { T2 } \\
\text { T3 } \\
\text { T4 }\end{array}$ & $\begin{array}{l}490.73 \\
732.3 \\
445.69 \\
621.33\end{array}$ & $\begin{array}{l}1.38 \\
-3.69 \\
-0.66 \\
-0.88\end{array}$ & $\begin{array}{l}{[-0.02,0.16]} \\
{[-0.24,-0.07]} \\
{[-0.15,0.07]} \\
{[-0.15,0.05]}\end{array}$ & $\begin{array}{l}0.17 \\
<\mathbf{0 . 0 0 1} \\
0.51 \\
0.38\end{array}$ \\
\hline $\begin{array}{l}\text { OY6 } \ell_{0} \text { T1 } \\
\text { T2 } \\
\text { T3 } \\
\text { T4 }\end{array}$ & $\begin{array}{l}272.14 \\
709.83 \\
370.74 \\
302.96\end{array}$ & $\begin{array}{l}-0.17 \\
-1.98 \\
-0.74 \\
1.44\end{array}$ & $\begin{array}{l}{[-0.05,0.04]} \\
{[-0.04,-0.0001]} \\
{[-0.04,0.02]} \\
{[-0.008,0.05]}\end{array}$ & $\begin{array}{l}0.87 \\
0.04 \\
0.46 \\
0.15\end{array}$ \\
\hline $\begin{array}{l}\text { OY6 } \ell_{0.25} \mathrm{~T} 1 \\
\text { T2 } \\
\text { T3 } \\
\text { T4 }\end{array}$ & $\begin{array}{l}291.61 \\
193.73 \\
282.08 \\
332.73\end{array}$ & $\begin{array}{l}-0.17 \\
-0.06 \\
-0.7 \\
-2.89\end{array}$ & $\begin{array}{l}{[-0.04,0.03]} \\
{[-0.05,0.05]} \\
{[-0.05,0.02]} \\
{[-0.09,-0.02]}\end{array}$ & $\begin{array}{l}0.86 \\
0.95 \\
0.49 \\
\mathbf{0 . 0 0 4}\end{array}$ \\
\hline $\begin{array}{l}\text { OY6 } \ell_{0.5} \mathrm{~T} 1 \\
\text { T2 } \\
\text { T3 } \\
\text { T4 }\end{array}$ & $\begin{array}{l}563.81 \\
589.17 \\
354.14 \\
313.60\end{array}$ & $\begin{array}{l}-4.47 \\
-0.05 \\
0.25 \\
0.54\end{array}$ & $\begin{array}{l}{[-0.14,-0.05]} \\
{[-0.03,0.04]} \\
{[-0.04,0.06]} \\
{[-0.03,0.06]}\end{array}$ & $\begin{array}{l}<\mathbf{0 . 0 0 1} \\
0.95 \\
0.8 \\
0.59\end{array}$ \\
\hline $\begin{array}{l}\text { OY6 } \ell_{0.75} \mathrm{~T} 1 \\
\text { T2 } \\
\text { T3 } \\
\text { T4 }\end{array}$ & $\begin{array}{l}712.11 \\
992.88 \\
482.95 \\
591.18\end{array}$ & $\begin{array}{l}-2.57 \\
-1.53 \\
-2.66 \\
-5.96\end{array}$ & $\begin{array}{l}{[-0.12,-0.02]} \\
{[-0.07,-0.009]} \\
{[-0.13,-0.02]} \\
{[-0.21,-0.11]}\end{array}$ & $\begin{array}{l}\mathbf{0 . 0 1} \\
0.13 \\
0.008 \\
<\mathbf{0 . 0 0 1}\end{array}$ \\
\hline $\begin{array}{l}\text { OY6 } \ell_{1.25} \mathrm{~T} 1 \\
\text { T2 } \\
\text { T3 } \\
\text { T4 }\end{array}$ & $\begin{array}{l}490.46 \\
708.37 \\
376.92 \\
232.27\end{array}$ & $\begin{array}{l}1.5 \\
1.87 \\
-0.59 \\
-1.66\end{array}$ & $\begin{array}{l}{[-0.02,0.16]} \\
{[-0.004,0.18]} \\
{[-0.15,0.08]} \\
{[-0.24,0.02]}\end{array}$ & $\begin{array}{l}0.15 \\
0.06 \\
0.55 \\
0.09\end{array}$ \\
\hline $\begin{array}{l}\text { OY6 } \ell_{1.75} \mathrm{~T} 1 \\
\text { T2 } \\
\text { T3 } \\
\text { T4 }\end{array}$ & $\begin{array}{l}408.3 \\
624.575 \\
439.12 \\
126.23\end{array}$ & $\begin{array}{l}13.25 \\
18.71 \\
9.13 \\
6.34\end{array}$ & $\begin{array}{l}{[0.67,0.90]} \\
{[0.88,1.10]} \\
{[0.41,0.63]} \\
{[0.42,0.81]}\end{array}$ & $\begin{array}{l}<0.001 \\
<0.001 \\
<0.001 \\
<0.001\end{array}$ \\
\hline
\end{tabular}

Table 3-3: Modified $t$ test for the mean-shifted swimming velocity $w_{m s}$ versus the swimming velocity $w_{s}$. The null hypothesis states that $\mu_{\text {shift }}=\mu_{\text {rel }}$ while the alternate hypothesis states that these means are not equal. Significance level is $\alpha=0.05 / 4=0.0125$ (with the Bonferroni correction). 


\begin{tabular}{|l|l|l|l|l|l|}
\hline Source & SS & $d f$ & MS & $F$ & $P$ \\
\hline \hline OY5 & & & & & \\
Turbulence level & 0.043 & 4 & 0.01 & 5.67 & $\mathbf{0 . 0 0 8}$ \\
Treatment order & 0.001 & 3 & $<0.001$ & 0.24 & 0.866 \\
Error & 0.022 & 12 & 0.002 & & \\
Total & 0.067 & 19 & & & \\
& & & & & \\
\hline \hline OY6 & & & & & \\
Turbulence level & 0.01 & 5 & 0.002 & 0.37 & 0.864 \\
Treatment order & 0.009 & 3 & 0.003 & 0.55 & 0.656 \\
Error & 0.079 & 15 & 0.005 & & \\
Total & 0.097 & 23 & & & \\
\hline
\end{tabular}

Table 3-4: Two-way ANOVA comparing the effects of turbulence level versus treatment order on larval swimming velocity. The null hypotheses state that average larval swimming velocities are equal across turbulence levels and independent of treatment order, while the alternate hypotheses state that these factors change average larval swimming velocity. Significance level is $\alpha=0.05$.

\subsubsection{Vertical swimming velocity in turbulence}

In OY5, larvae showed a significant swimming response to turbulence in the two-way ANOVA (Table 3-4), swimming upward on average at higher turbulence levels. The post-hoc multiple comparison test (Fig. 3-5) demonstrated that upward larval swimming increased significantly in moderate turbulence $\left(\ell_{0.5}\right.$ and $\left.\ell_{0.75}\right)$ when compared to swimming in still water and low turbulence $\left(\ell_{0}\right.$ and $\left.\ell_{0.25}\right)$. Further, the $t$ tests (Table 3-5, Fig. 3-5) demonstrated that swimming velocities in moderate to high turbulence $\left(\ell_{0.75}\right.$ and $\left.\ell_{1.25}\right)$ were significantly greater than zero. Treatment order had no effect on swimming velocity in either OY5 or OY6 (Table 3-4), indicating that exposure history did not influence the larval response to a particular treatment level.

OY6 data had greater inter-trial variability and no significant swimming response to turbulence was detected (Table 3-4), but several general patterns emerged in both experiments (dotted lines, Fig. 3-5). In almost all the trials, larval swimming velocities were near zero in low turbulence $\left(\ell_{0}\right.$ and $\left.\ell_{0.25}\right)$, with both positive and negative mean velocities observed. At higher turbulence levels $\left(\ell_{0.5}\right.$ and higher $)$ mean swimming velocities were greater in magnitude, and predominantly positive (upward swimming). At the highest turbulence level $\left(\ell_{1.75}, \mathrm{OY} 6\right)$, larvae demonstrated more flexibility in their vertical directionality, with larvae swimming upward in two trials and downward in two others. 


\begin{tabular}{|l|l|l|l|l|}
\hline Turbulence level & $d f$ & $t$ stat & $95 \%$ CI on $\left|\mu_{1}-\mu_{2}\right|$ & $p$-value \\
\hline \hline OY5 $\ell_{0}$ & 3 & -0.23 & {$[-0.06,0.05]$} & 0.58 \\
$\ell_{0.25}$ & 3 & 0.94 & {$[-0.01,0.02]$} & 0.2 \\
$\ell_{0.5}$ & 3 & 2.94 & {$[-0.05,0.24]$} & 0.03 \\
$\ell_{0.75}$ & 3 & 4.91 & {$[0.01,0.19]$} & $\mathbf{0 . 0 0 8}$ \\
$\ell_{1.25}$ & 3 & 4.63 & {$[0.02,0.16]$} & $\mathbf{0 . 0 0 9}$ \\
\hline
\end{tabular}

Table 3-5: Results of $t$ test on OY5 data to determine the effects of turbulence level on upward larval swimming. Trials are grouped in each turbulence level and the null hypothesis states that the mean vertical larval swimming velocity is less than or equal to zero, and the alternate hypothesis states that it is positive (that is, larvae swim upwards). Significance level is $\alpha=0.05 / 5=0.01$ (with the Bonferroni correction).

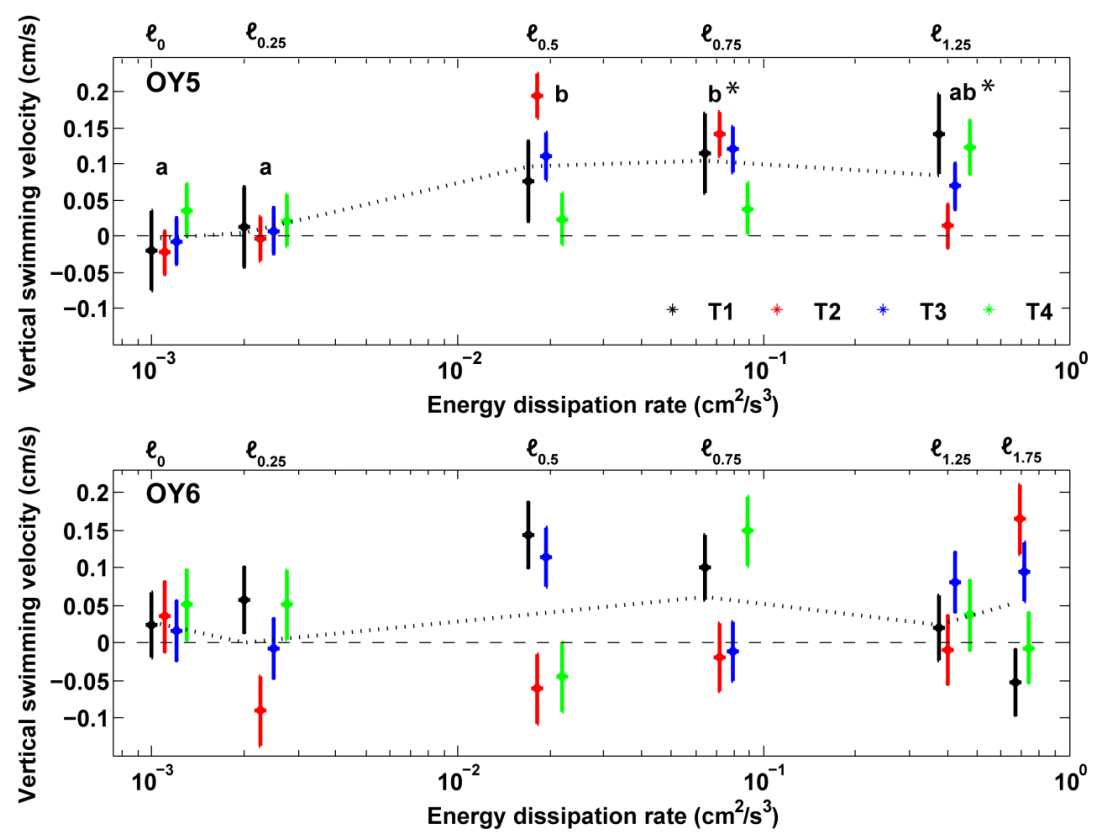

Figure 3-5: Mean instantaneous swimming velocity $w_{s}$ with respect to energy dissipation rate $\varepsilon$, for all four trials of both OY 5 and OY6. Error bars are 95\% confidence intervals as computed from pooled sample variance for each trial, and the dashed line indicates zero swimming velocity. Letters denote turbulence levels for which swimming velocities differ significantly, from the post-hoc multiple comparison test. Asterisks above grouped trials denote significantly positive (upward) swimming velocities, as determined by $t$ tests (Table 3-5). Dotted lines indicate grouped trial means, to illustrate qualitative similarities between OY5 and OY6.

\subsubsection{Dive frequency in turbulence}

Dive frequency (Fig. 3-6, Table 3-6) changed significantly with turbulence level in both experiments OY5 and OY6. The $\chi^{2}$ binomial comparison test on the contingency table 
(Table 3-6) gave $d f=4, \chi_{Y}^{2}=150.49$, and $p<0.001$ for OY5 data and $d f=4, \chi_{Y}^{2}=$ 138.19 , and $p<0.001$ for OY6 data. Dive frequency decreased with increasing turbulence level, where a subdivision of the contingency table led to demonstrated that the onset of statistically significant changes in dive frequency occurred between levels $\ell_{0}$ and $\ell_{0.25}$ in OY5 $\left(d f=2, \chi_{Y}^{2}=12.02, p<0.001\right)$, and between $\ell_{0.25}$ and $\ell_{0.5}$ in OY6 $\left(d f=2, \chi_{Y}^{2}=9.73\right.$, $p=0.002)$.

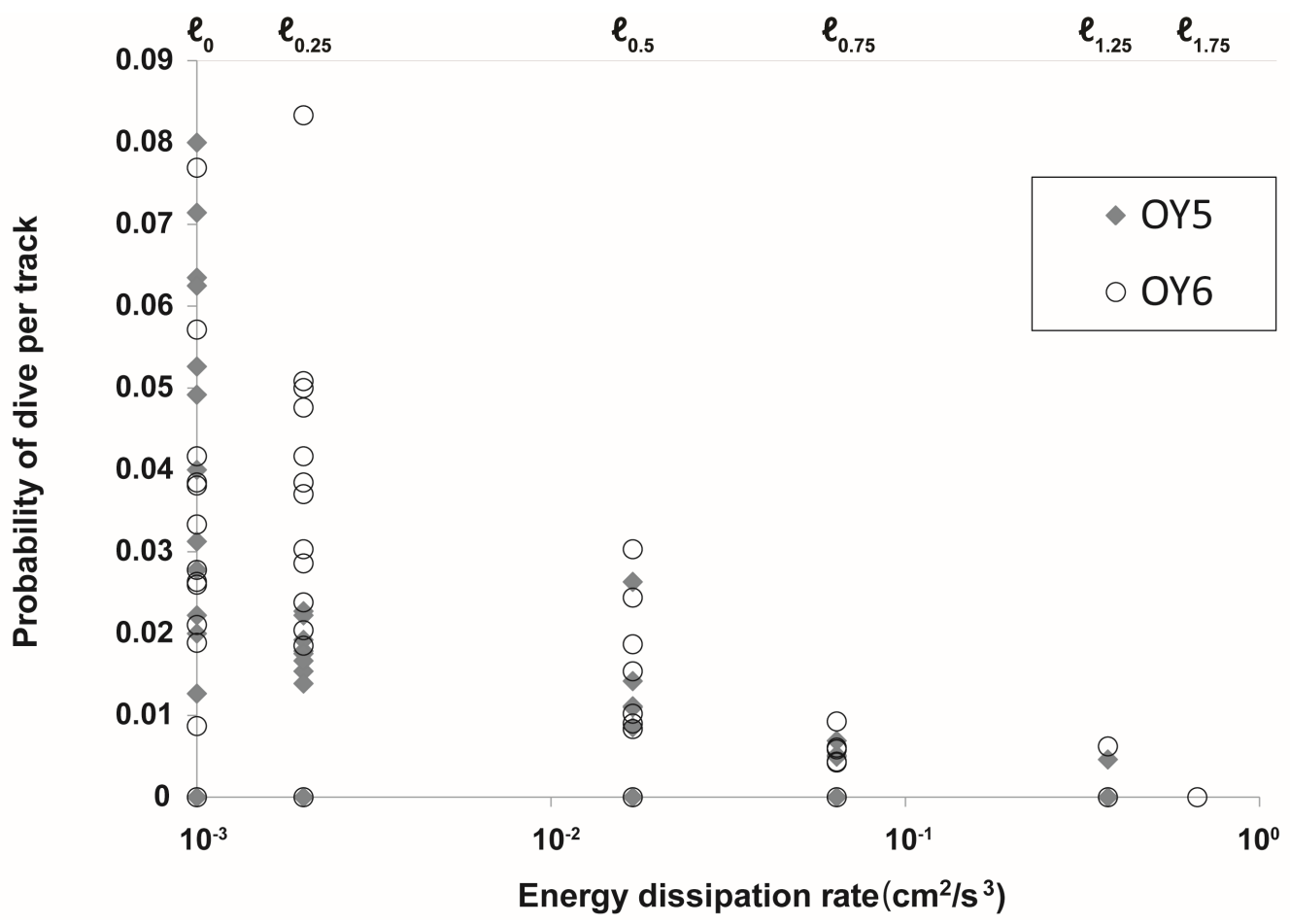

Figure 3-6: Probability of dive (frequency of dives divided by number of larval trajectories) with respect to energy dissipation rate, for each dataset, where OY5 datasets are represented by a grey diamond, and each dataset of OY6 is represented by an open circle.

\subsection{Discussion}

Swimming behavior of oyster larvae varied with turbulence level, but not in the way we had hypothesized. Larval swimming velocities were weak in low turbulence and generally became stronger and upward in moderate turbulence. While high inter-trial variation in one experiment (OY6) precluded a statistically significant effect of turbulence on larval swimming, both experiments demonstrated qualitatively similar patterns. Upward swimming did 


\begin{tabular}{|l|l|l|l|l|l|l|}
\hline Turb. Level & $\ell_{0}$ & $\ell_{0.25}$ & $\ell_{0.5}$ & $\ell_{0.75}$ & $\ell_{1.25}$ & $\ell_{1.75}$ \\
\hline \hline OY5 & & & & & & \\
Dive & 24 & 8 & 7 & 4 & 1 & - \\
No dive & 666 & 893 & 1274 & 2893 & 3620 & - \\
& & & & & & \\
OY6 & & & & & & \\
Dive & 23 & 17 & 10 & 5 & 1 & 0 \\
No Dive & 1058 & 797 & 1640 & 2578 & 2726 & 3604 \\
\hline
\end{tabular}

Table 3-6: Contingency table for $\chi^{2}$ binomial comparison test for significance of dive frequency. Columns under turbulence levels contain the number of larval trajectories containing dives and the number of tracks not containing dives. The null hypothesis states that the proportion of larvae which dive (compared to non-divers) is independent of turbulence level, while the alternate hypothesis states that said proportion changes with turbulence level. Significance level is $\alpha=0.05$.

not cease at an upper turbulence threshold. The dive response did not increase in frequency with increasing turbulence and did not cause downward movement of the population as a whole. In fact, the dive response in larvae decreased significantly in increasing turbulence, so our initial hypothesis of increasing turbulence as a dive trigger is not supported by the results. Larvae demonstrated the ability to maintain upward swimming velocities even in high turbulence, but the behaviour demonstrated some variability as average downward swimming was also observed at the highest turbulence level tested.

We further found that simultaneous subtraction of local flow from larval movement was necessary to isolate larval behaviour, insofar as a mean-shifted larval velocity distribution could not reliably capture the mean and variance of the more accurate swimming velocity distribution. That is, a temporally and spatially averaged flow velocity does not adequately approximate local flow subtraction near individual larvae, confirming the necessity of a PIV-type approach to simultaneous larval tracking and flow quantification. This result has considerable implications for the design of larval behaviour experiments.

A surprising result of our experiments is the observation of continued larval swimming even in highly turbulent flow. We expected larvae to cease swimming in high turbulence, and exhibit negative velocities consistent with sinking [25]. Instead, even in high turbulence, larvae typically continued to swim upward. This suggests that turbulence may not be a settlement trigger for oyster larvae, given their tendency to swim up in highly turbulent flow. Further, the upward motion is an active response of larvae, and not a result of passive advection in turbulence. Studies of weakly inertial particle motion in turbulent flow 
demonstrate that turbulence leads to small modifications of the average fall velocity (e.g. $[108,109,110])$, but does not cause the particles to move upward on average.

Another unexpected result of our experiments is the unwillingness or inability of larvae to dive in even moderately turbulent flows. The dive response is not uncommon when no flow is induced in the tank; it appears in up to $8 \%$ of larval tracks in a trial (Fig. 3-6). However, the dive frequency decreases (and in the case of the OY5 experiment, significantly) when any flow is induced in the tank.

The propensity of larvae to actively swim upward in high turbulence and not exhibit a dive response is especially interesting in the context of the results of Finelli and Wethey [96]. In an open channel flow, they observed a dive response in approximately $4 \%$ of larvae filmed near a bottom boundary layer. Working with their reported shear velocity, camera height, and water level height, we estimated an energy dissipation rate of $\varepsilon \approx 0.37 \mathrm{~cm}^{2}$ $\mathrm{s}^{-3}$, following the methodology of Fuchs et al. [94]. This dissipation rate is comparable to $\ell_{1.25}$ from our experiment, where we observed only two dives in 6348 larval tracks. This wide discrepancy in dive frequency suggests the existence of a physical trigger for the dive response present in a channel flow that was absent in our experiment. Though estimated dissipation rates were comparable between their experiment and $\ell_{1.25}$ of our experiment, their flume tank had horizontal mean flow of $4.5 \mathrm{~cm} \mathrm{~s}^{-1}$. The presence of a relatively large horizontal mean flow indicates strong vertical shear approaching the bottom of the flume, suggesting that larvae may dive in response to vertical shear. Alternately, the presence of the vertical mean flows in the grid-stirred tank at higher turbulence levels may have suppressed the larval dive response, as larvae may have chosen to swim against a downward mean flow to maintain vertical position (observed experimentally for cyprid larvae in [55]). Larval swimming and diving responses to local components of turbulence which they may sense (acceleration, strain, and vorticity), are a subject of current investigation.

Oyster larvae continued to swim upward in turbulent flow of energy dissipation rates comparable to estuarine and calm surf zone conditions. If turbulence is a trigger to settlement, we have yet to observe a threshold turbulent energy level high enough to cause these larvae to consistently stop swimming and sink or dive out of the water column. Further experiments with more strongly turbulent flows within reasonable field conditions may yet yield this threshold. It is also possible that turbulence induced by vertical grid movement is insufficient to trigger a settlement or downward swimming response in competent larvae, 
though downward swimming has been reported for larger competent larvae in a turbulence tank study [27]. Our larvae were in an early phase of competency, as they had not reached the larger sizes $(300 \mu \mathrm{m})$ of settling larvae reported from other studies (e.g.,[102]). Larger larvae may be more inclined to move downward as they develop heavier shells towards the end of competency, due either to energetic constraints (more energy needed to keep shell aloft), or developmental limits (i.e., they become desperate larvae [111]). Further, the specific turbulent cues to which larvae respond are not yet well understood: larvae approaching nearshore environments may react to highly turbulent flows with strong shear (as in the flume of Finelli \& Wethey [96]), or possibly a combination of turbulence and a chemical cue are required to induce larval settlement responses (see for instance [99]). Our results raise the intriguing question of how larval responses to turbulent flows may change through the competent-to-settle period.

A useful technical result obtained from this study is the demonstration that adjusting absolute larval velocity distributions by subtracting mean, non-local flow appears inadequate for capturing larval swimming metrics, especially the variances of relative velocity distributions. The variances in the mean-shifted swimming velocity distributions increased dramatically with increasing turbulence level, likely due to enhanced random variability in the experimental flow. A similar increase in variance was also observed in Fuchs et al. [25], although their mean flow subtraction methodology differed somewhat from that presented here. Our mean flow velocities were considerably greater than those reported in Fuchs et al. [25], as they observed maximal mean flows of $-0.7 \mathrm{~cm} \mathrm{~s}^{-1}$, while ours were $-2.6 \mathrm{~cm}$ $\mathrm{s}^{-1}$, though this discrepancy may be due to the time interval over which the mean flow was calculated. Roy et al. [52] also reported sporadic considerable mean flows in their tank, occurring at far lower turbulence levels than used in our experiment. Mean flows appear inherent to the vertically-oscillating grid tank design, and as the mean-shifted velocity correction was especially inaccurate at high turbulence, we conclude that local flow subtraction is an unavoidable necessity at high turbulence. The spatial and temporal gradients in flow velocity at high turbulence also impede efforts to separate average flow from larval swimming velocities, further indicating the necessity of local flow subtraction. Finally, flow subtraction is necessary to identify behaviors such as the dive response, as they occur on an individual level and are too transient to be observed clearly in swimming velocity distributions in all but the lowest turbulence levels (e.g. Fig. 3-4 c). To determine how often the diving 
behavior occurs, however, one requires the isolated swimming velocity time series of each individual larva.

In this study, we determined that oyster larvae, unlike previously studied mollusc species, do not sink in experimental turbulent flows comparable to coastal settlement zones, and in fact continue to actively swim. Further, we found that previous methods for estimating larval swimming velocities may be inadequate to distinguish behavior from advection in fluid flow. Because larval behavior may have profound effects on supply and successful settlement to the benthos, we must continue to carefully isolate behavioral effects from flow in larval invertebrates. For oyster larvae specifically, further work remains to determine a potential settlement response to hydrodynamic cues. Larval swimming responses to faster flows, vertical shear, and combined turbulent and chemical cues are the subject of future investigation.

\subsection{Acknowledgements}

Thanks to Skylar Bayer, Meredith White, and Stace Beaulieu for discussions of larval behaviour and culture, Susan Mills for larval acquisition and culture, and experimental assistance, Shawn Arellano for discussions of larval tracking and behaviour in flow, and Heidi Fuchs for helpful discussions during the inception of the project. Thanks also to Joseph Fitzgerald for discussions of larval behavior, tracking, and data analysis., and to Vicke Starczak for suggestions on statistical analysis. Thanks also to three anonymous reviewers for helpful manuscript comments and suggestions. We gratefully acknowledge the culturing expertise of the Aquaculture Research Facility in Dennis, MA, who supplied the larval oysters used in this study. This work was supported by NSF grant OCE-0850419, grants from WHOI Coastal Ocean Institute, discretionary WHOI funds to purchase the IR laser and high-speed camera, and a WHOI Ocean Life Fellowship to LSM. 


\section{Chapter 4}

\section{Isolating the hydrodynamic triggers of the dive response in eastern oyster}

\section{larvae}

\subsection{Abstract}

${ }^{1}$ Understanding the behavior of larval invertebrates during planktonic and settlement phases remains an open and intriguing problem in larval ecology. Larvae modify their vertical swimming behavior in response to water column cues in order to feed, avoid predators, and search for settlement sites. The larval eastern oyster (Crassostrea virginica) can descend in the water column via active downward swimming, sinking, or "diving", which is a flick and retraction of the ciliated velum to propel a transient downward acceleration. Diving may play an important role in active settlement, since diving larvae move rapidly downward in the water column and may regulate their proximity to suitable settlement sites. Alternatively, it may function as a predator-avoidance escape mechanism. We examined potential hydrodynamic triggers to this behavior by observing larval oysters in a grid-stirred turbulence tank. Larval swimming was recorded for two turbulence intensities and flow properties around each larva were measured using particle image velocimetry. The statistics of flow properties likely to be sensed by larvae (fluid acceleration, deformation, vorticity, and angular acceleration) were

\footnotetext{
${ }^{1}$ Originally published as "JD Wheeler, KR Helfrich, EJ Anderson, and LS Mullineaux (2015). Isolating the hydrodynamic triggers of the dive response in eastern oyster larvae. Limnology and Oceanography, 60: 1332-1343." Reproduced here under the terms of the Creative Commons Attribution License, which permits use, distribution and reproduction in any medium, provided the original work is properly cited.
} 
compared between diving and non-diving larvae. Our analyses showed that diving larvae experienced high average flow accelerations in short time intervals (approximately 1-2 seconds) prior to dive onset, while accelerations experienced by non-diving larvae were significantly lower. Further, the probability that larvae dove increased with the fluid acceleration they experienced. These results indicate that oyster larvae actively respond to hydrodynamic signals in the local flow field, which has ecological implications for settlement and predator avoidance.

\subsection{Introduction}

Many marine invertebrates have a planktonic larval dispersal period before settling to the seafloor as adults. Our understanding of how larval behavior may influence dispersal and transport across a range of spatial scales is limited [45], and larval responses to a variety of physical, chemical, and biological cues remain ongoing areas of research. Larval swimming can be impacted by turbulent flow fields, especially in the turbulent bottom boundary layer as larvae move towards the substratum (e.g. [91, 92]). However, the impact of turbulent flow on the behavior of individual larvae is not well characterized due to technical challenges in simultaneously quantifying larval swimming and the motion of the surrounding flow field. Recent advances $[27,28]$ are now making such studies feasible.

Small swimming organisms in a turbulent ocean experience a complex fluid environment, and may potentially respond to different components of ambient flow conditions, such as temporal velocity gradients (acceleration), spatial velocity gradients governing fluid deformation and rotation (strain rate and vorticity, respectively), and temporal vorticity gradients (angular acceleration). Rapid behavioral responses to local flow conditions are better studied for zooplankton than for larvae: threshold flow deformation has been observed to trigger escape responses in copepods [112] as well as multiple protists [113]. Acceleration, meanwhile, has not been observed to produce a similar response, though both acceleration and deformation are strong components of the suction flow fields produced by feeding predators $[112,113,114]$. In vortical flows, small organisms (ranging from bacteria to larvae) tilt and reorient, a response that has been attributed to a physical mechanism involving the balance of viscous and gravitational torques acting on the organism (see for example $[115,116,57]$. In this study, we focus on the larvae of the eastern oyster, Crassostrea virginica, to increase 
our understanding of rapid behavioral responses of marine invertebrate larvae, and bivalves particularly, to flow conditions that they might experience in the field.

We chose oyster larvae for this study because they exhibit intriguing swimming behaviors in turbulent flows characteristic of coastal benthic habitats. They swim using a ciliated velum and so control their own swimming direction in still water, likely sensing their orientation and swimming direction with respect to gravity using a statocyst structure [102]. A specific behavior of interest in oyster larvae is a response known as "dive-bombing" or "diving" $[96,28]$. Herein, we consider diving as a transient response occurring over timescales of approximately one second, where larvae abruptly accelerate downward, achieving speeds up to $1 \mathrm{~cm} \mathrm{~s}^{-1}$, or approximately 50 body lengths $\mathrm{s}^{-1}$, which is distinct from the sustained slower downward swimming behavior defined as diving in Fuchs et al. [27]. Diving, as we have defined it, has been observed in a moderately turbulent channel flow [96], and in low turbulence induced by a grid-stirred tank [28]. The cue or cues triggering the onset of the dive response are not well understood: some population-level estimates of larval swimming velocity in flow suggest that downward swimming increases in high turbulence [27], while others suggest that larvae persist in upward swimming in high turbulence, and further, that the dive response disappears in highly turbulent flow [28]. As larval swimming responses in turbulence appear to be highly variable at the population level, we seek to identify specific triggers experienced consistently by larvae immediately prior to dive onset. It is important to identify these cues because through diving, a larva can rapidly displace itself downward through the water column. This behavior may therefore impact larval supply to the benthos, as diving may help larvae avoid predators and/or identify and approach suitable settlement sites.

Larvae settling into oyster reefs and other complex benthic structures experience a complex fluid environment which may impact settlement patterns (e.g. [117, 91, 118]). Current field research on oyster reefs suggests a link between oyster larval settlement patterns and turbulent flow over regions of settlement. Whitman and Reidenbach [97] observed that turbulent drag and shear fields were considerably higher over live oyster reefs than mud flats and restoration reefs made of broken oyster or whelk shells. Larvae were observed to settle preferentially on oyster reefs, followed by whelk shell restoration sites, then oyster shell restoration sites, and not at all on mud flats. Settlement patterns suggest that flow fields generated by rough relief and low levels of turbulence in interstitial spaces may abet larval 
recruitment. Because oyster larvae display a dive response in turbulent conditions, we want to determine whether or not larvae dive in response to local hydromechanical cues in the turbulent flow field, such as flow acceleration, deformation, vorticity, or angular acceleration.

When transitioning out of the water column to the benthos, oyster larvae experience turbulent flow fields that may induce rapid downward diving responses. In this study, we actively quantify the diving response observed in two turbulence regimes, and determine which (if any) local hydromechanical signals induce the response, as well as the response timescales. Further, we use a Bayesian approach to calculate probabilities of larval diving conditioned on specified local hydromechanical conditions (e.g. the probability of a larva diving, supposing it has experienced a specified flow acceleration for a specified length of time). This relationship may be useful for understanding the ecological implications of larval responses in specific field conditions, and for the integration of behavior into larval models. We determine these diving triggers by identifying diving larvae and their local flow conditions in experimentally generated grid-stirred turbulence, then comparing the conditions experienced by diving and non-diving larvae as they move through the turbulent fluid environment.

\subsection{Methods}

\subsubsection{Experimental organism and larval culturing}

Crassostrea virginica, the eastern oyster, is a mollusc species native to the North Atlantic. Adults inhabit coastal shallow waters and broadcast spawn into the plankton, where larvae reside as free-swimming planktotrophs for 2-3 weeks [6]. Larvae entering the final planktonic stage, referred to as pediveligers, develop a foot and commonly a pronounced eyespot which are used in aquacultural practice to denote competency to settle [103].

We obtained such competent larvae from the Aquaculture Research Corporation in Dennis, Massachusetts, United States of America, in three separate spawns in the summers of 2011, 2012, and 2013. All spawns were retained prior to experiments in identical culture conditions: $3 \mu \mathrm{m}$-filtered, aerated seawater at ambient field temperature $\left(20-22^{\circ} \mathrm{C}\right)$ and salinity (33 psu), in covered 16 L plastic buckets. Larvae were kept at low densities to minimize interactions ( 3000 larvae L-1) and fed a suspension of haptophyte Isochrysis sp. once

per day (375 mL filtered seawater with $9 \times 105$ cells $\mathrm{mL}^{-1}$.) Larvae were given a minimum 
period of 8 hours to acclimate post-transport from the aquaculture facility, and used for experiments within two days of competency onset. A representative sample of larvae from the 2013 spawn were measured and examined for eyespots prior to their use in experiments: average larval width (perpendicular to hinge) was $277 \mu \mathrm{m}$, average height (parallel to hinge) was $264 \mu \mathrm{m}$, and percentage of larvae with eyespots was $>80 \%$.

\subsubsection{Experimental set-up}

The turbulence tank used in the experiments (see [28] for schematic) consists of a $180 \mathrm{~L}$ plexiglass tank $(44.5 \times 44.5 \times 90 \mathrm{~cm})$ with two horizontal grid structures set equidistant from the center of the tank, connected by vertical rods in each corner. The grid structures are made from $1 \mathrm{~cm} \times 1 \mathrm{~cm}$ plexiglass bars spaced $5 \mathrm{~cm}$ apart. Both grids are connected to a motor above the tank by a vertical rod, which drives a simultaneous vertical oscillation in the grids. The oscillation amplitude is $5 \mathrm{~cm}$ and the oscillation frequency is specified by the user to induce flow fields of different turbulence intensity.

In the analysis described in this study, the larvae were subjected to two turbulence levels, hereafter referred to as "unforced" and "forced" regimes: the first regime has no flow induced in the tank (i.e. the grid frequency is $0 \mathrm{~Hz}$ ) and the second regime has low forcing conditions with a grid frequency of $0.25 \mathrm{~Hz}$. The forced regime has an estimated energy dissipation rate of $2 \times 10^{-3} \mathrm{~cm}^{2} \mathrm{~s}^{-3}$, and has Kolmogorov and integral length scales of $0.14 \mathrm{~cm}$ and 3.02 $\mathrm{cm}$, respectively, roughly comparable to calm field conditions in tidal channels and estuarine flows [76]. Note that although the grid was not operating in the unforced case, there was weak turbulent flow in the tank due to residual motions and possibly convection. The original experiments additionally subjected larvae to more highly turbulent flow conditions with dissipation rates ranging from $0.017 \mathrm{~cm}^{2} \mathrm{~s}^{-3}$ in a moderate turbulence regime to 0.667 $\mathrm{cm}^{2} \mathrm{~s}^{-3}$ in the most highly turbulent regime, and associated Kolmogorov and integral length scales ranging from 0.08 to $0.03 \mathrm{~cm}$ and 3.64 to $3.59 \mathrm{~cm}$, respectively. These regimes were not examined in our present study because the larval diving behavior disappears in more highly turbulent flow (see [28]).

A vertical cross-section in the center of the tank was illuminated by a pulsed near-infrared laser (Oxford Lasers, Firefly $300 \mathrm{~W}, 1000 \mathrm{~Hz}, 808 \mathrm{~nm}$ ) in a plane approximately $1 \mathrm{~mm}$ thick. A high-speed monochrome camera (Photron Fastcam SA3, $1024 \times 1024$ pixel resolution) was trained perpendicularly to the laser sheet, recording a $\sim 3 \times 3 \mathrm{~cm}$ two-dimensional field 
of view (FOV).
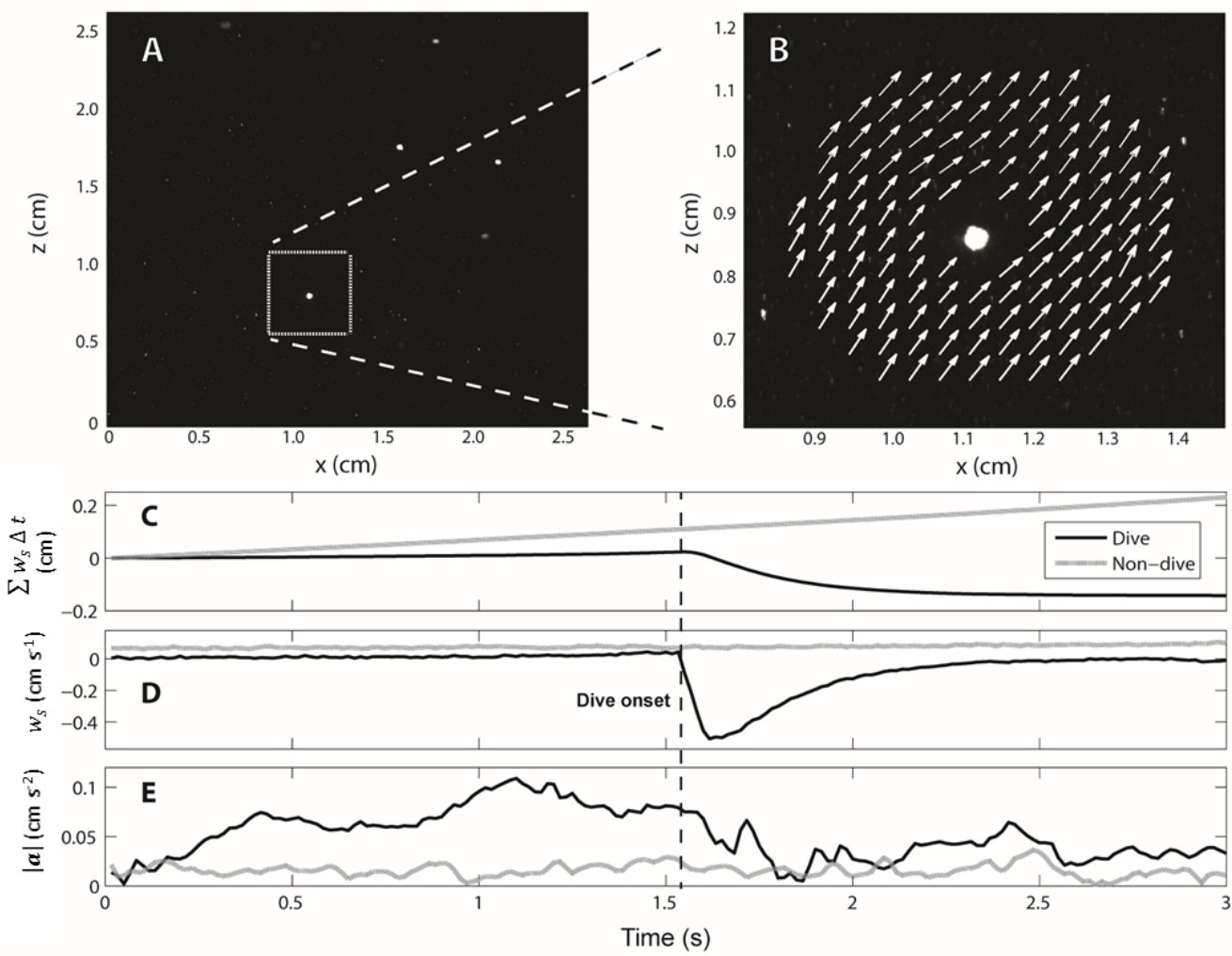

Figure 4-1: (A) Sample image from field of view in the turbulence tank: larvae are bright white spots and polystyrene passive particles are small dim white specks. (B) Close up of individual larva (white spot) overlaid with annulus of local flow velocity field (white arrows) estimated using PIV. (C-E) Sample time series of diving (black curve) versus non-diving larva (grey curve), where the vertical black dashed line denotes dive onset time: vertical displacement due to larval swimming $\sum w_{s} \Delta t(\mathrm{C})$, vertical swimming velocity $w_{s}(\mathrm{D})$, and flow acceleration magnitude $|\mathbf{a}|$ experienced by each larva $(\mathrm{E})$.

The tank was maintained in an environmental chamber of fixed temperature $\left(20^{\circ} \mathrm{C}\right)$ and filled with surface seawater filtered to particle size $<1 \mu \mathrm{m}$. Larvae were gently introduced into the tank using a beaker to densities of $0.5-0.62$ larvae $\mathrm{mL}^{-1}$. The tank was subsequently seeded with a $2.5 \mathrm{~mL}$ suspension of neutrally buoyant polystyrene passive particles (3.0-3.4 $\mu \mathrm{m}$ diameter, $1.05 \mathrm{~g} \mathrm{~cm}^{-3}$ density, $5 \%$ weight by volume, Spherotech, Lake Forest, Illinois, USA) to a density of $\sim 4.2 \times 10^{4}$ particles $\mathrm{mL}^{-1}$ for flow quantification by particle image velocimetry (PIV). Preliminary experiments showed no effects of these artificial particles on larval swimming in still water, when compared to both swimming in control filtered seawater and seawater seeded with natural Isochrysis algae (of roughly comparable size and 
concentration), leading us to conclude that artificial particles could be used in turbulence experiments without affecting behavior.

Larval behavior was recorded for 5-6 separate 45 second intervals at 60 fps (with the number of intervals depending on the spawn and the turbulence level). These intervals were separated in time by approximately 5 minutes each to transfer images from the camera to the computer as TIFF files (e.g. Fig. 4-1 A). Experiments were conducted under identical conditions over three separate two day periods in the summers of 2011, 2012, and 2013, corresponding to three separate spawns. Larvae were subjected to multiple randomly ordered turbulence levels, though only the two lowest turbulence regimes were examined in the present study. Turbulence treatment order has no observed effect on larval swimming velocity [28], so eliminating measurements from these higher turbulence levels should not affect our results. Separate batches of larvae were also pooled for this analysis. Analyses of mean vertical swimming velocities in higher turbulence regimes, and separated by larval batches, are presented for the 2011 and 2012 data in Wheeler et al. [28].

\subsubsection{Larval tracking and local flow subtraction}

The following methodology for isolating larval swimming velocity from advection in the local flow field was presented in Wheeler et al. [28] and is summarized here, with the added refinement of interpolating local flow velocities to larval positions. First, larvae were identified by the following method: all TIFF files were imported into LabVIEW 2010 (National Instruments) and average background intensity was subtracted. Larval centroid positions ( $x$ and $z$ coordinates) were identified using a fixed threshold particle size and intensity and recorded along with larval size, in the frame which they appeared.

Second, observed larval trajectories were computed using an in-house MATLAB script which tracked identified larvae from frame to frame according to a subsequent-frame tolerance distance radius set by the user. Larval trajectories were truncated by five frames at both the beginning and end of the trajectories due to uncertainties in centroid estimates in cases where larvae passed laterally into and out of the focal plane, which caused larvae to appear diffuse and out of focus. Instantaneous observed larval velocities, denoted

$\mathbf{u}_{o b s}=\left[u_{o b s}, w_{o b s}\right]$ for each larva, were computed using a central difference scheme of larval centroid position in time, so that the velocity is defined centered in time between the two images. 
Third, fluid velocity fields in the FOV were quantified using PIV imaging software LaVision DaVis (v.7.2). All TIFF files were imported into the software and velocity fields were computed using correlations (default FFT with Whittaker reconstruction) of most likely passive particle positions from frame to frame, using $16 \times 16$ pixel interrogation windows (with 7-8 particles per window, not distinguishable by eye in Figs. 4-1 A-B). This process yielded two $64 \times 64$ spatial grids of horizontal and vertical flow velocity for each time step, corresponding to a grid spacing of $0.039-0.046 \mathrm{~cm}$ (varied slightly by spawn).

Fourth, fluid velocities local to larvae were subtracted from observed larval velocities to obtain larval swimming velocities by the following method. The velocity fields estimated by PIV were imported and converted to MATLAB data files and velocity vectors in an annulus around each larva were used to estimate the fluid velocity at the larval position at each time step. The radius of the annulus changed dynamically for each larva: the inner radius was the sum of the maximum individual larval radius and the grid spacing of the PIV data (16 pixels), and the outer radius was four times greater than the inner radius (Fig. 4-1 B). The inner radius of the annulus masked the larval presence in the PIV data, which might otherwise contaminate the PIV analysis for fluid velocity. The velocity data in the annulus were fit to a two-dimensional, second-order Taylor series function by least-squares. The flow velocity $\mathbf{u}=[u, w]$ local to a larva was then obtained by evaluating the function at the larval centroid position. This interpolated fluid velocity was subtracted from the observed larval velocity at that time step to obtain the larval swimming velocity $\mathbf{u}_{s}=\left[u_{s}, w_{s}\right]$. For each larva,

$$
\mathbf{u}_{s}=\mathbf{u}_{o b s}-\mathbf{u}
$$

\subsubsection{Identification of the dive response}

The dive response was initially observed by eye in experimental footage and in individual larval vertical swimming velocity time series, where it was characterized by a rapid drop to high downward swimming velocities, followed by a slow deceleration over the span of several seconds to near-zero vertical swimming velocity. We described a larva as diving if it performed downward accelerations of at least $3.0 \mathrm{~cm} \mathrm{~s}^{-2}$ (approximately 150 body lengths $\mathrm{s}^{-2}$ ) for minimally 2 time steps $(1 / 30 \mathrm{~s})$ and achieved negative vertical swimming velocities

of at least $-0.4 \mathrm{~cm} \mathrm{~s}^{-1}$. These thresholds in vertical swimming acceleration and velocity were used to separate diving larvae from non-diving larvae in the subsequent analysis (example 
difference between diving and non-diving larvae velocity time series, Fig. 4-1 D).

\subsubsection{Hydromechanical parameters detectable by larvae}

In this section, we propose a suite of hydromechanical cues in the turbulent flow that are likely to be detectable by larvae. Because larvae can be divided into divers and non-divers, relevant potential cues experienced by these two groups (Fig. 4-1 E) can then be compared for statistical differences. Following Kiørboe and Visser [119], one may isolate the various aspects of a turbulent flow to which a larva might respond. Potentially relevant hydromechanical triggers are fluid acceleration, deformation (strain rate), rotation (vorticity), and angular acceleration. Given a flow velocity $\mathbf{u}$ local to a larva having swimming velocity $\mathbf{u}_{s}$, on any given time step, we can calculate the following acceleration, strain rate, vorticity, and angular acceleration fields.

Acceleration measures the rate of change in fluid velocity and could potentially be perceived by a larva through its statocyst structure: a calcareous statolith would be displaced into the wall of the statocyst cavity due to inertia in an accelerating flow [120, 27]. To characterize the temporal changes in flow velocity near an individual larva, we use the magnitude

of the two-dimensional acceleration of the fluid flow following the larval position [121] (see the Supplemental Material, Section 4.7.1, for a derivation):

$$
|\mathbf{a}|=\left|\frac{\partial \mathbf{u}}{\partial t}+\left(\mathbf{u}+\mathbf{u}_{s}\right) \cdot \nabla \mathbf{u}\right|
$$

We use acceleration magnitude, with magnitude denoted by $|\cdot|$, as a hydromechanical metric in order to incorporate both dimensions of the acceleration vector. This acceleration metric excludes the acceleration that a larva experiences due to its own swimming motion, accounting only for the acceleration the larva experiences due to the local flow field. Larval swimming velocity $\mathbf{u}_{s}$ is present in $|\mathbf{a}|$ because both larval swimming and flow velocity contribute to larval position, hence the inclusion of both in the advection term. If larvae perceive acceleration using a statocyst, they would feel the total acceleration from both the flow and their own swimming (see Supplemental Material, Section 4.7.1). However, we focus on the externally-imposed fluid acceleration because it is independent of all larval behavior: this simplifies the interpretation of our results, as we do not conflate the larval responses to internally-imposed and externally-imposed motion. 
In practice, the flow acceleration above is calculated by interpolating flow velocity to the larval position at each time step, then using a central difference scheme to compute the temporal derivative along the larval path. While the acceleration magnitude used in this analysis uses only the two known dimensions $(x, z)$ available from our PIV set-up, the unknown $y$-acceleration component will be similar to that of $x$, due to tank and forcing symmetries. We estimated a three-dimensional acceleration magnitude by doubling the $x$ acceleration component and found that the two and three-dimensional fluid acceleration estimates yield similar statistical results, so we report only the two-dimensional results in the subsequent sections.

The velocity gradients in a fluid flow lead to shear stresses on the surface of any object or fluid parcel in that flow. The net effect of these shear stresses can be to strain (i.e. deform) and rotate the object or fluid parcel. The strain rate (quantified using the rate of strain tensor) determines how a fluid parcel is stretched or sheared in different spatial dimensions, and could potentially be detected by a larva at sufficiently high signal strength by a deformation of cilia along the velum. The rotation rate (quantified using the vorticity) is likely detectable through a larva's statocyst structure [120], as the statolith is displaced and rolls steadily along the statocyst cavity wall, imposing a centrifugal force.

Strain rate is quantified in a three dimensional flow by the symmetric strain rate tensor $e_{i j}$, elements of which describe the deformation of the flow along two axes. Because we have only two dimensions of velocity data, the full strain rate tensor cannot be computed, and we are restricted to the examination of three of the elements of the tensor: the shear strain rate $e_{x z}$ and the normal strain rates $e_{x x}$ and $e_{z z}$. We use the two-dimensional shear strain rate magnitude at the larval position:

$$
\left|e_{x z}\right|=\left|\frac{1}{2}\left(\frac{\partial u}{\partial z}+\frac{\partial w}{\partial x}\right)\right|
$$

This metric represents the shearing, or deformation, of a fluid parcel in the focal plane, and is calculated using flow velocities local to the larval position. We use the magnitude of the shear strain rate because the sign of this term simply governs the direction in which the shear deformation occurs, and we do not expect larvae to recognize or respond to this directionality. 
Normal strain rates are

$$
e_{x x}=\frac{\partial u}{\partial x}
$$

and

$$
e_{z z}=\frac{\partial w}{\partial z}
$$

where these quantities measure how fluid is stretched in the $x$ and $z$ dimensions, respectively, calculated local to the larval position at each time step. Unlike the shear strain rate, the signs of the normal strain rates are retained; positive normal strain rates indicate divergence in the specified spatial dimension, while negative normal strain rates indicate convergence in the specified spatial dimension, and these are physically distinct phenomena. For all strain rates, the spatial derivatives are calculated at the fluid velocity points in the annulus around each larva and then interpolated to the larval position using the method described for the velocity field in the local flow subtraction section.

Vorticity measures the rotation of a fluid parcel, and is likely detectable through a larva's statocyst structure, as described above. Vorticity is a three dimensional vector for a three dimensional flow, with each element describing the rotation of the fluid normal to a plane described by the other two dimensions. Because we have only two dimensions of velocity data, we are restricted to using the vorticity element normal to the focal plane as our vorticity metric:

$$
\left|\omega_{y}\right|=\left|\frac{\partial w}{\partial x}-\frac{\partial u}{\partial z}\right|
$$

The vorticity is calculated local to larval position at each time step, with spatial derivatives calculated as described above for the strain rate metrics. Similarly to shear strain rate, we define our vorticity metric by the magnitude of the vorticity element: the sign of vorticity denotes the direction of rotation of the local fluid (clockwise versus anti-clockwise), which we do not expect the larvae to distinguish. In a simple parallel shear flow, vorticity is equal to the velocity gradient in a single direction, and we use vorticity in this study because it generalizes the shear metric commonly reported in simpler flows [119]. Similar to the acceleration term defined above, this vorticity term accounts only for the fluid rotation around the larva and not the larva's own rotation term. The larval rotation term is not considered in this analysis; as above, the rationale is to separate external forcing imposed by the fluid from the internal forcing of the larva's own swimming motion. In addition, larval 
rotation (unlike larval acceleration) cannot be reliably measured in this set of experiments.

Angular acceleration measures the rate of rotation of a fluid parcel, and may be detectable in the larval statocyst structure through the onset of statolith motion along the statocyst wall. To characterize the temporal changes in flow vorticity near an individual larva, we compute the magnitude of the angular acceleration of the fluid flow following the larval position:

$$
|\alpha|=\left|\frac{\partial \omega_{y}}{\partial t}+\left(\mathbf{u}+\mathbf{u}_{s}\right) \cdot \nabla \omega_{y}\right|
$$

In practice, the angular acceleration is calculated by interpolating flow vorticity to the larval position at each time step, then using a central difference scheme to compute the temporal derivative along the larval path. To avoid confusion, in the following analysis and discussion, acceleration always refers to a, the rate of change of fluid velocity following larval paths, while angular acceleration specifically will be used to refer to $\alpha$, the rate of change of fluid vorticity following larval paths.

\subsubsection{Statistical analysis}

In this study, one of our objectives was to determine differences in hydromechanical parameters (flow acceleration, normal and shear strain rates, vorticity, and angular acceleration) experienced by diving larvae and non-diving larvae. To determine this, we calculated mean hydromechanical parameters experienced by all diving larvae in a set temporal interval immediately prior to dive onset, and mean hydromechanical parameters in the same temporal interval (randomly selected in the individual larval trajectory) for non-diving larvae. We used means instead of maxima, as using mean values in short time intervals allowed us to capture peak hydromechanical parameter values while filtering out PIV noise that distorts the maxima. A randomly subsampled group of non-diving larvae were then selected to compare to the diving larvae, so that the sample size in both groups would be identical. Two conditional probability distributions were then constructed for comparative purposes: $P(T \mid$ larva dives $)$ and $P(T \mid$ larva does not dive $)$ for each mean hydromechanical parameter $T$.

The distributions of mean hydromechanical parameters experienced by diving larvae and non-diving larvae were then compared statistically using the following methods. If $T$ was strictly non-negative (i.e. all magnitude terms) we used a non-parametric 2-sided Wilcoxon 
rank sum test to compare the medians of the diving versus non-diving distributions. If the distributions were drawn from both positive and negative values, we used a modified 2-tailed $t$-test (Welch's approximate $t$-test statistic and Satterthwaite's approximation for the degrees of freedom) to compare the means of the distributions instead.

If a parameter was found to differ significantly between diving and non-diving larvae, both distributions were compared to the background distribution of the hydromechanical parameter, $P(T)$, which was determined by computing $T$ through four fixed spatial points in the FOV over the three experiments (over comparable spatial and temporal scales to which $T$ was computed for the larvae). The comparisons of diving, non-diving, and background $T$ distributions were carried out using a non-parametric Kruskal-Wallis test. A multiple comparison test was subsequently carried out to identify whether hydromechanical parameters experienced by diving and/or non-diving larvae differed significantly from the average parameter values in the background flow. All statistical tests were carried out using MATLAB.

For any hydromechanical parameter which differed significantly between diving and nondiving larvae, the conditional probability of diving given a specified mean parameter value was calculated using Bayes theorem:

$$
P(\text { larva dives } \mid T)=\frac{P(\text { larva dives }) \cdot P(T \mid \text { larva dives })}{P(T)} \text {. }
$$

The probability of larval diving, $P$ (larva dives), is the number of diving larval trajectories divided by the total number of trajectories observed, while $P(T \mid$ larva dives $)$ and $P(T)$ are described above. The conditional probability of larval diving given a mean hydromechanical parameter value, $P($ larva dives $\mid T)$, is an ecologically relevant function as it predicts larval behavior in response to specific environmental conditions.

A $95 \%$ confidence interval for this conditional probability was computed by summing in quadrature the independent confidence intervals from each term in the equation. Confidence intervals for $P$ (larva dives $\mid T)$ and $P(T)$ were estimated by bootstrapping the distributions and directly computing the confidence interval for each value of $T$. The confidence interval for the scalar $P$ (larva dives) was computed using the Clopper-Pearson method for binomial confidence intervals as the diving probability is a probability of success in a binomial trial (i.e. diving vs. non-diving). 


\subsection{Results}

\subsubsection{Identification of dive response}

Using our quantitative definition of diving, we found that 82 larvae (of 874 total larvae) dove at least once during their observed trajectory in the unforced regime, and 57 larvae (of 1019 total larvae) dove at least once in the forced regime. We overlaid the diving trajectories aligned by dive onset time in the unforced regime (Fig. 4-2) to identify similarities in diving trajectories, and found similar timescales in the downward acceleration for all larvae, on the order of $0.1 \mathrm{~s}$. Larvae reached peak downward velocities ranging from -0.5 to $-0.7 \mathrm{~cm}$ $\mathrm{s}^{-1}$ (slightly less than passive terminal fall velocity, indicative of continued active control). Larvae decelerated to zero velocity in approximately one second. Prior to dive onset, larvae engaged in a range of vertical velocities, centered near zero, but both upward and downward swimming were observed, suggesting that larvae had no fixed pre-dive behavior. As larvae decelerated from the dive and resumed a more constant vertical velocity, they exhibited a similar range of vertical velocities, indicating that larvae also had no fixed post-dive behavior. Vertical displacement from a single dive was of order $10^{-1} \mathrm{~cm}$, or approximately 4 body lengths, and comparable to the Kolmogorov scale, the length scale of the smallest eddies in the forced regime.

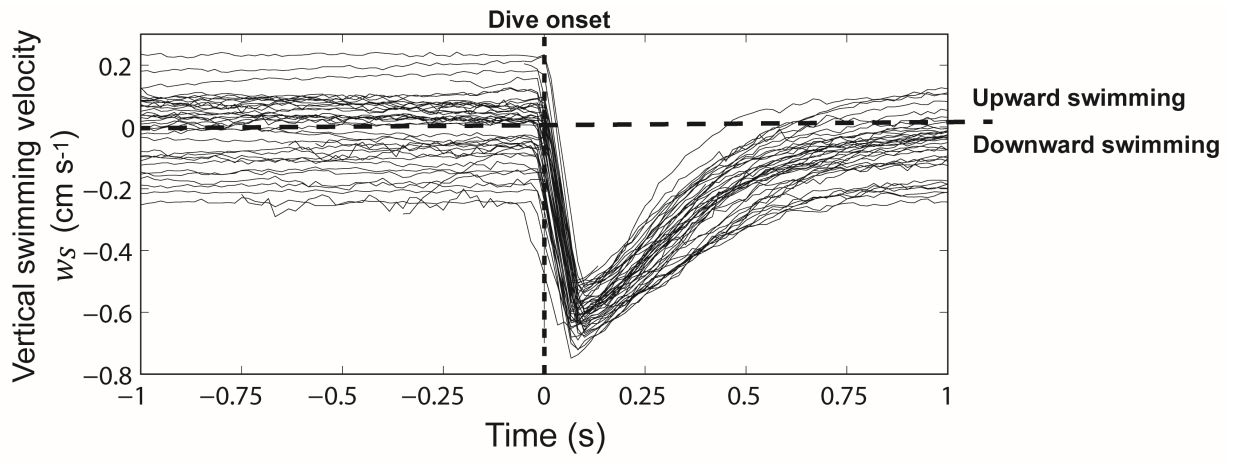

Figure 4-2: Diving larval vertical swimming velocity time series in the unforced regime, aligned by dive onset time. Larvae display strong uniformity in time spent accelerating downward, maximum downward velocity, and time spent decelerating out of the dive. Larvae exhibit a range of vertical swimming velocities prior to dive onset. 

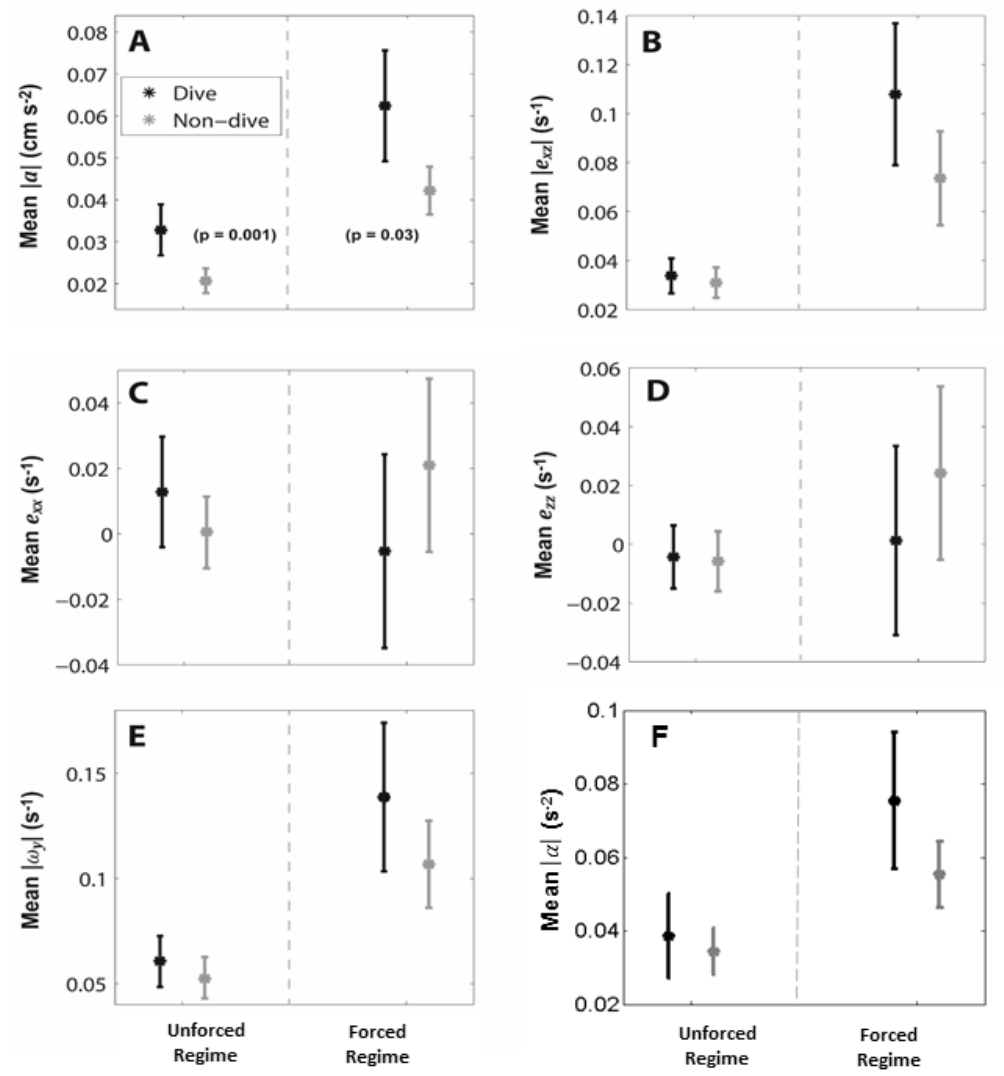

Figure 4-3: Values of hydromechanical parameters (mean and $95 \%$ confidence intervals) for diving larvae (black) and non-diving larvae (grey) in unforced and forced regimes. Values are calculated in a $1.66 \mathrm{~s}$ time interval prior to the dive onset in diving larvae, and a randomly selected $1.66 \mathrm{~s}$ time interval in the trajectories of non-diving larvae. Sample sizes are $n=82$ for both groups in the unforced regime, and $n=57$ in the forced regime. (A) Mean acceleration magnitude $|\mathbf{a}|$ is significantly different between diving and non diving larvae for both turbulence regimes (see Table 4-1). (B) Mean shear strain rate magnitude $\left|e_{x z}\right|$ experienced by diving and non-diving larvae is not significantly different in either turbulence regime (Supplemental Material Table S2). (C-D) Mean horizontal and vertical normal strain rates $e_{x x}$ and $e_{z z}$ experienced by diving and non-diving larvae are not significantly different in either turbulence regime (Supplemental Material Table S1). (E) Mean vorticity magnitude $\left|\omega_{y}\right|$ experienced by diving and non-diving larvae is not significantly different in either turbulence regime (Supplemental Material Table S2) (F) Mean angular acceleration magnitude $|\alpha|$ experienced by diving and non-diving larvae is not significantly different in either turbulence regime (Supplemental Material Table S2).

\subsubsection{Hydromechanical parameters triggering the dive response}

A range of temporal intervals prior to the dive onset was investigated, from $0.33 \mathrm{~s}$ to $3 \mathrm{~s}$, in intervals of $0.33 \mathrm{~s}$ (see Supplemental Materials) to identify potential reaction timescales 
for diving larvae. A hydromechanical parameter was considered to be a consistent trigger to the dive response only if 1 ) it differed significantly between diving and non-diving larvae in the specified temporal interval, and 2) this significant response held in both flow regimes, unforced and forced, for identical temporal intervals.

Diving larvae consistently experienced significantly higher mean acceleration than nondiving larvae. In the unforced regime, mean accelerations were significantly higher for diving larvae in the $1,1.33,1.66$, and $2 \mathrm{~s}$ time intervals and intermittently significant for longer time intervals (Table 4-1, Fig. 4-3 A). In the forced regime, mean accelerations were significantly higher for diving larvae in all time intervals from $1.33 \mathrm{~s}$ to $3 \mathrm{~s}$ prior to the dive onset (Table 4-1, Fig. 4-3 A). The intersection of these temporal intervals is $1.33-2.33 \mathrm{~s}$, representing the consistent response range in which diving larvae experienced significantly higher acceleration than non-diving larvae. For subsequent analyses presented in the main text, we used a central point of this interval, $1.66 \mathrm{~s}$ prior to dive onset, as the averaging window and denote the mean acceleration experienced by a larva in this interval as $|\mathbf{a}|_{1.66}$.

No other hydromechanical parameter differed significantly prior to dive onset between diving and non-diving larvae (Figs. 4-3 B-F, Supplemental Material Tables S1 and S2) in contrast to acceleration (Fig. 4-3 A, Table 4-1). That is, none of shear deformation, normal deformation (horizontal or vertical), vorticity, or angular acceleration induced a diving response in larvae in any temporal window examined.

Flow accelerations experienced by diving and non-diving larvae in the $1.66 \mathrm{~s}$ interval were then compared to background acceleration fields (Fig. 4-4). These three distributions of flow acceleration, $P\left(|\mathbf{a}|_{1.66} \mid\right.$ larva dives $), P\left(|\mathbf{a}|_{1.66} \mid\right.$ larva does not dive $)$, and $P\left(|\mathbf{a}|_{1.66}\right)$, were significantly different in the unforced regime (Table 4-2). A post-hoc multiple comparison test of these distributions demonstrated that diving larvae experienced significantly higher average flow accelerations than both non-diving larvae and the average background acceleration. Non-diving larvae experienced flow accelerations that were indistinguishable from the background acceleration. In the forced regime, a similar pattern was observed: diving larvae experienced higher accelerations than did non-diving larvae, as well as higher accelerations than those occurring in the background flow. However, the result in this regime was non-significant (Table 4-2), likely due to the smaller sample size of dives and lower power of the multi-way comparison. 


\begin{tabular}{|l|l|l|l|l|}
\hline $\begin{array}{l}\text { Time interval prior } \\
\text { to dive onset }(\mathrm{s})\end{array}$ & Turbulence regime & Rank sum & $\mathrm{z}$ & $p$-value \\
\hline \hline 0.33 & Unforced regime & 6314 & 1.84 & 0.06 \\
0.66 & $\varepsilon \rightarrow 0 \mathrm{~cm}^{2} \mathrm{~s}^{-3}$ & 6270 & 1.67 & 0.09 \\
1.00 & & 6490 & 2.48 & $\mathbf{0 . 0 1}$ \\
1.33 & & 6735 & 3.39 & $<\mathbf{0 . 0 0 1}$ \\
1.66 & & 6697 & 3.25 & $\mathbf{0 . 0 0 1}$ \\
2.00 & & 6406 & 2.17 & $\mathbf{0 . 0 2}$ \\
2.33 & & 6148 & 1.22 & 0.21 \\
2.66 & & 6626 & 2.99 & $\mathbf{0 . 0 0 2}$ \\
3.00 & & 6318 & 1.88 & 0.06 \\
& & & & \\
\hline \hline 0.33 & Forced regime & 3095 & 1.63 & 0.1 \\
0.66 & $\varepsilon=10^{-3} \mathrm{~cm}^{2} \mathrm{~s}^{-3}$ & 3076 & 1.51 & 0.13 \\
1.00 & & 3015 & 1.13 & 0.25 \\
1.33 & & 3166 & 2.08 & $\mathbf{0 . 0 3}$ \\
1.66 & & 3164 & 2.07 & $\mathbf{0 . 0 3}$ \\
2.00 & & 3325 & 3.08 & $\mathbf{0 . 0 0 2}$ \\
2.33 & & 3232 & 2.5 & $\mathbf{0 . 0 1}$ \\
2.66 & & 3144 & 1.94 & $\mathbf{0 . 0 5}$ \\
3.00 & & 3244 & 2.57 & $\mathbf{0 . 0 0 9}$ \\
\hline
\end{tabular}

Table 4-1: Wilcoxon rank sum test comparing medians of mean acceleration distributions experienced by diving versus non-diving larvae, where means are computed in the stated window prior to dive onset. The null hypothesis states that medians $M_{d}=M_{n d}$ while the alternate hypothesis states that they differ. Significance level is $\alpha=0.05$. The medians of mean acceleration distributions are significantly higher for diving larvae than non-diving larvae in both flow regimes, given at least a $1.33 \mathrm{~s}$ window over which local acceleration is averaged. 


\begin{tabular}{|l|l|l|l|l|l|}
\hline Source & SS & $d f$ & MS & $\chi^{2}$ & $\mathrm{P}$ \\
\hline \hline Unforced regime & & & & & \\
Group & $5.54 \times 10^{4}$ & 2 & $2.77 \times 10^{4}$ & 12.40 & $\mathbf{0 . 0 0 2}$ \\
Error & $9.71 \times 10^{5}$ & 228 & $4.26 \times 10^{4}$ & & \\
Total & $102 \times 10^{6}$ & 230 & & & \\
\hline \hline Forced regime & & & & & \\
Group & $9.76 \times 10^{3}$ & 2 & $4.88 \times 10^{3}$ & 4.38 & 0.11 \\
Error & $3.51 \times 10^{5}$ & 160 & $2.19 \times 10^{3}$ & & \\
Total & $3.60 \times 10^{5}$ & 162 & & & \\
\hline
\end{tabular}

Table 4-2: Kruskal-Wallis test comparing median average accelerations experienced by the following three groups: diving larvae in a $1.66 \mathrm{~s}$ window prior to dive onset, non-diving larvae in a random $1.66 \mathrm{~s}$ window, and four fixed spatial points over all three experiments in a random $1.66 \mathrm{~s}$ window. The null hypothesis states that medians of all three mean acceleration distributions are equal, and the alternate hypothesis states that the mean accelerations experienced by these groups are different. Significance level is $\alpha=0.05$.
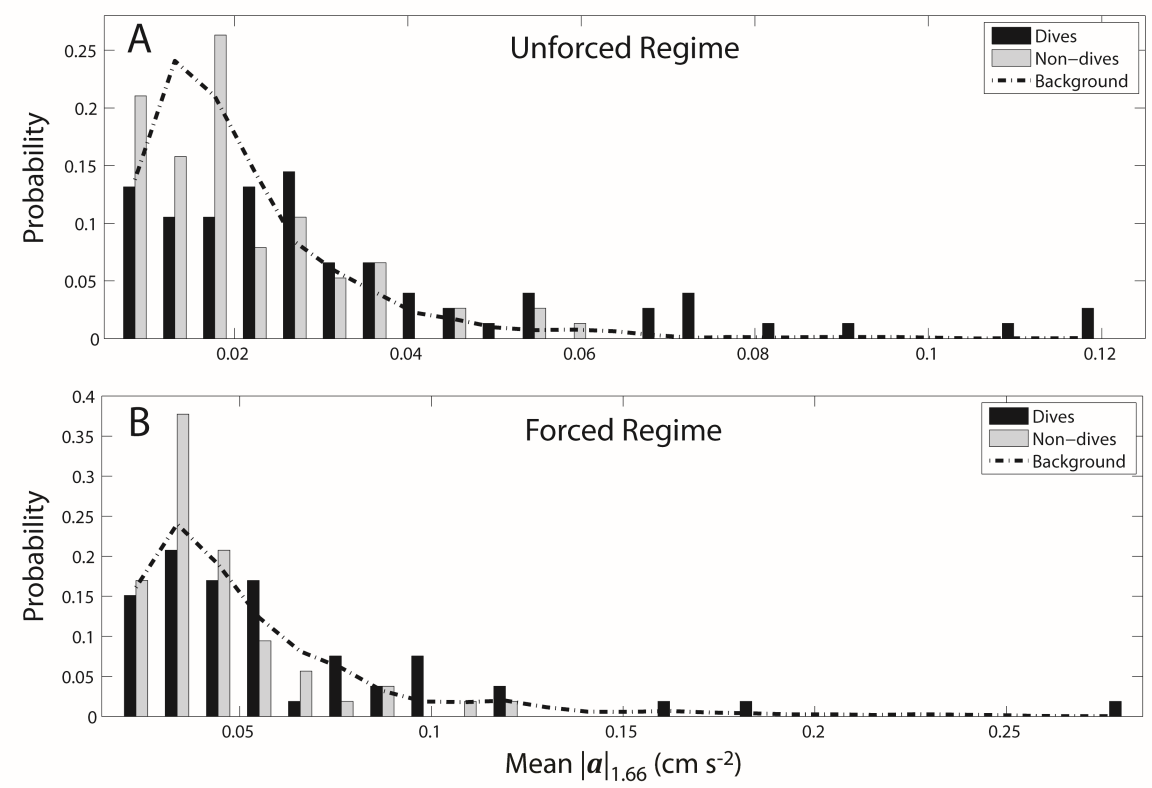

Figure 4-4: Probability distributions of mean flow acceleration magnitude experienced by larvae in a $1.66 \mathrm{~s}$ time interval (prior to dives for diving larvae, randomly selected for non-diving larvae), in unforced (A) and forced (B) regimes, respectively. The black bar distributions are those of diving larvae, $P\left(|\mathbf{a}|_{1.66} \mid\right.$ larva dives $)$, the grey bar distributions are those of non-diving larvae, $P\left(|\mathbf{a}|_{1.66} \mid\right.$ larva does not dive), and the black dashed curves are background mean acceleration magnitudes $P\left(|\mathbf{a}|_{1.66}\right)$. Note the different acceleration scales in unforced and forced regimes.

These distributions were then used to compute $P$ (larva dives $\left.\left.|| \mathbf{a}\right|_{1.66}\right)$, the conditional probability that larvae dove for a given acceleration averaged over the $1.66 \mathrm{~s}$ pre-dive window 
in the unforced regime (Fig. 4-5). The positive relationship between this probability and the acceleration demonstrates that diving became a more probable response as mean fluid acceleration experienced by larvae increased. The bounds on the $95 \%$ confidence intervals increased for high acceleration values due to the rarity of high acceleration events, which likely also accounted for overestimates of the conditional diving probability (i.e. greater than 1) for high accelerations. The computation is omitted for the forced regime as the large decrease in number of dives observed renders estimates much more uncertain.

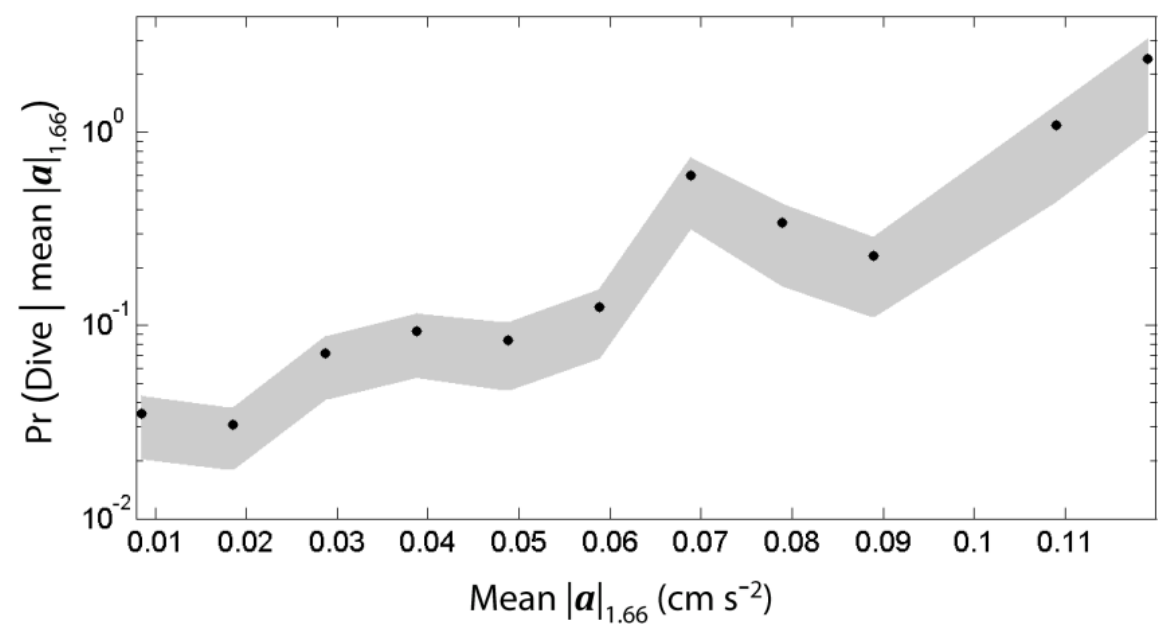

Figure 4-5: Probability of larval dive conditioned on $|\mathbf{a}|_{1.66}$, the local mean acceleration field (averaged over $1.66 \mathrm{~s}$ window), i.e. $P$ (larva dives $|\mathbf{a}|_{1.66}$ ), for the unforced regime. Larvae were more likely to dive when they encountered higher local flow acceleration. Shaded grey region represents the $95 \%$ confidence interval for all mean accelerations.

\subsection{Discussion}

Comparisons of flow fields experienced by diving and non-diving larvae strongly support a conclusion that flow acceleration triggers the dive response in oyster larvae. Diving larvae experienced significantly higher mean fluid accelerations than did non-diving larvae during a short period leading up to the dive onset in both turbulence regimes. The other candidate hydromechanical parameters did not differ significantly between diving and non-diving larvae: none of mean normal strain rates, shear strain rate, vorticity, or angular acceleration triggered the dive response. An examination of diving in the central $1.66 \mathrm{~s}$ response window demonstrated that not only did diving larvae experience higher accelerations than 
non-diving larvae, but that these accelerations were anomalously high compared to the background (significantly so in the unforced regime). The correspondence between probability of diving and increasing fluid acceleration further reinforces the interpretation that diving is triggered by acceleration. Further, the time interval over which the threshold mean acceleration was experienced was important for triggering the dive response. When acceleration was averaged over temporal windows shorter than $1.33 \mathrm{~s}$, higher acceleration did not appear to induce diving preferentially. This analysis suggests that the reaction timescale of the larvae to the fluid acceleration field they experience was at least $1.33 \mathrm{~s}$. A lack of pattern in timescales longer than $2 \mathrm{~s}$ suggests that the larvae are responding to an acceleration event, roughly $1.5 \mathrm{~s}$ before the dive, rather than to mean acceleration over a longer interval.

The observation that a mean acceleration of $0.035 \mathrm{~cm} \mathrm{~s}^{-2}$ triggered a dive in the unforced case, but not in forced case, indicates that the required threshold acceleration changes with the turbulence level. In the low-forcing regime, an average acceleration of $0.06 \mathrm{~cm} \mathrm{~s}^{-1}$ triggered a dive, while non-diving larvae experienced mean accelerations of $0.04 \mathrm{~cm} \mathrm{~s}^{-2}$. This result suggests that larvae become conditioned to the flow regime in which they find themselves, and the dive response is triggered by anomalously high accelerations compared to the background acceleration. This interpretation is supported by the finding that the accelerations experienced by diving larvae were significantly higher than both non-diving larvae and the background field. In a previous study [28], the dive response was found to disappear entirely in highly turbulent flow conditions (having energy dissipation rates greater than $10^{-1} \mathrm{~cm}^{2} \mathrm{~s}^{-3}$ ). While our experimental results do not provide a complete explanation for this disappearance, we offer several possibilities. First, larvae may simply stop reacting to an acceleration trigger above a certain threshold which occurs in the higher turbulence regimes. Second, recall that larvae respond to anomalously high accelerations within a turbulence level, and this threshold increases with turbulence intensity, at least in unforced and low forcing conditions. The frequency with which larvae encounter sufficiently high acceleration anomalies in more turbulent regimes may be lower, which would explain the lack of diving in these regimes. However, we cannot quantify the diving threshold accelerations for these higher flow regimes (beyond supposing the thresholds are greater than that observed in our low flow forced regime), and as such, this explanation for the lack of diving in high turbulence remains speculative. Alternatively, it is possible that the experimental set-up precluded detection of dives because larvae are advected quickly 
in more highly turbulent flow. It is possible that it becomes more difficult to observe the diving response because larvae remain in the FOV for shorter time periods (though more larvae are observed in higher turbulence regimes).

The dive response for all observed larvae was highly uniform in terms of acceleration and deceleration timescales (Fig. 4-2), and the response is predictable based on fluid acceleration through the conditional probability $P$ (larva dives || $\mathbf{a} \mid)$. These characteristics make the dive response well suited for inclusion into individual based models of larval behavior in complex flow fields (see for instance [122]). Such models would be very useful for testing whether diving affects settlement success in simulated turbulent flow fields over rough bottom topography. The strong uniformity of the dive further suggests that the response, once instigated, is regulated by biomechanical constraints, as all larvae emerge from the dive and resume swimming on comparable timescales. In this way, the diving response triggered by acceleration may differ from the sinking response to waterborne chemical cues observed in the larval sea slug Phestilla sibogae [48]. These larvae retract velar lobes instantly in response to coral-conditioned seawater, and continue to sink unless the cue is absent on timescales of one second or longer. Our larvae, conversely, cease to dive after approximately 1 second regardless of local flow conditions. While the larvae are capable of diving multiple times in succession, their behavior appears distinct from the sustained sinking observed in P. sibogae larvae.

The effects of local environmental conditions on the behavior of mollusc larvae have been previously studied in a few species with varying results. Two bivalve larvae (Crassostrea gigas and Mytilus edulis) exposed to horizontal suction flow demonstrated no discernible swimming response as they approached a suction tube [123], a flow that would have a strong acceleration signal. However, the flow fields experienced by these larvae were quantified in a separate experiment from the larval observations. This technique can make it difficult to isolate larval behavior [28], as small scale temporal and spatial variations in the flow field that larvae might experience are not captured. $P$. sibogae retract their velar lobes in response to mechanical stimulus [48], and potentially to local hydrodynamic conditions (M. Koehl, personal communication), as well as the potentially distinct response to chemical cues, as discussed above. The similarity of the response (retraction of ciliated swimming organ into a shell) in different mollusc groups suggests that larval diving in response to acceleration may be common to multiple species. 
A dive response when larvae are experiencing anomalously high accelerations could potentially be a beneficial strategy if they need to settle onto rough bottom surfaces, or to avoid predator feeding currents. We consider both possibilities, beginning with the ecological implications of diving as a settlement response. PIV measurements over rough topography in an oscillating flow tank have demonstrated that the highest accelerations occur up to 5 cm from the bottom, and decay rapidly farther above [124]. Further, simulated larvae in the PIV-measured flow experience peak accelerations of short duration that are much higher in magnitude than the mean values, much like the anomalously high accelerations experienced by the diving larvae in our study. The threshold accelerations experienced by larvae in our unforced and forced regimes are small compared to the fluid accelerations near the bottom reported by Pepper et al. [124], but may help larvae navigate downward through the water column at heights above $5 \mathrm{~cm}$ from the bottom. The dive response disappears in more highly turbulent flow regimes that more closely mimic the energetics of flow immediately above preferred settlement sites (e.g. [97]), which offers further evidence the dive response is likely to be employed by larvae higher in the water column.

Larvae could alternatively experience flow acceleration due to suction feeding flows from predators in the plankton $[112,113,114]$, or even from the feeding currents of adult oysters on reefs [123]. In this way, the dive could act as an escape response analogous to the jumping behavior of copepods (e.g. [125, 126]) or the rapid downward swimming of insect larvae and pupae (e.g. [127]) observed in the presence of predators. Larval dive responses to flow acceleration in the water column could thus increase larval supply to the seafloor, by either increasing the rate of downward flux, or decreasing the proportion lost to predators.

\subsection{Acknowledgements}

Thanks to Elaine Luo, Brenna McGann, Susan Mills, Andre Price, and Anthony Ritchie for assistance with larval acquisition, culture, experiments, and thanks to Anna Wargula for turbulence tank measurements. Thanks to Margaret Byron, Karen Chan, Joseph Fitzgerald, Mimi Koehl, and Evan Variano for helpful discussions throughout this project, and two anonymous reviewers for suggestions. We gratefully acknowledge the culturing expertise of the Aquaculture Research Corporation in Dennis, Massachusetts, who supplied the larval oysters used in this study. This work was supported by NSF grant OCE-0850419, NOAA 
Sea Grant NA14OAR4170074, grants from the WHOI Coastal Ocean Institute, discretionary WHOI funds, a WHOI Ocean Life Fellowship to LM, and a Grove City College Swezey Fellowship to EA. 


\subsection{Supplemental Material}

\subsubsection{Flow acceleration following larval trajectory}

The following section presents the derivation of $\mathbf{a}$, the flow acceleration following the path of a swimming larva. Suppose a larva swims with velocity $\mathbf{u}_{\mathbf{s}}(t)$ in a flow field having velocity $\mathbf{u}(x, t)$. We first calculate the total observed acceleration experienced by the larva, in terms of contributions from larval swimming and from the flow. In this scenario,

$$
\mathbf{u}_{o b s}(\mathbf{x}, t)=\mathbf{u}(\mathbf{x}, t)+\mathbf{u}_{\mathbf{s}}(t)
$$

is the observed velocity of the larva at time $t$ and position $\mathbf{x}$. At the initial position and time $\left(\mathbf{x}_{0}, t_{0}\right)$, the larva has an observed velocity of

$$
\mathbf{u}^{\mathbf{0}}{ }_{\text {obs }}=\mathbf{u}\left(\mathbf{x}_{\mathbf{0}}, t_{0}\right)+\mathbf{u}_{\mathbf{s}}\left(t_{0}\right) \equiv \mathbf{u}^{0}+\mathbf{u}_{\mathbf{s}}^{0}
$$

At time $t=t_{0}+\delta t$, the larva has an observed velocity of

$$
\mathbf{u}^{\mathbf{1}}{ }_{o b s}=\mathbf{u}\left(\mathbf{x}_{\mathbf{0}}+\delta \mathbf{x}, t_{0}+\delta t\right)+\mathbf{u}_{\mathbf{s}}\left(t_{0}+\delta t\right)
$$

where $\delta \mathbf{x}=\left(\mathbf{u}+\mathbf{u}_{\mathbf{s}}\right) \delta t$. To obtain the total observed acceleration $\frac{d^{2} \mathbf{x}}{d t^{2}} \equiv \mathbf{a}_{\mathrm{obs}}$,

$$
\begin{aligned}
\mathbf{a}_{\mathrm{obs}} & =\frac{\mathbf{u}_{\mathbf{1}}-\mathbf{u}_{\mathbf{0}}}{\delta t} \\
& =\frac{1}{\delta t}\left(\mathbf{u}^{0}+\delta \mathbf{x} \cdot \nabla \mathbf{u}+\delta t \frac{\partial \mathbf{u}}{\partial t}+\mathbf{u}_{\mathbf{s}}^{0}+\delta t \frac{d \mathbf{u}_{\mathbf{s}}}{d t}-\left(\mathbf{u}^{0}+\mathbf{u}_{\mathbf{s}}^{0}\right)\right) \\
& =\frac{1}{\delta t}\left(\delta \mathbf{x} \cdot \nabla \mathbf{u}+\delta t \frac{\partial \mathbf{u}}{\partial t}+\delta t \frac{d \mathbf{u}_{\mathbf{s}}}{d t}\right) \\
& =\frac{1}{\delta t}\left(\delta t\left(\mathbf{u}+\mathbf{u}_{\mathbf{s}}\right) \cdot \nabla \mathbf{u}+\delta t \frac{\partial \mathbf{u}}{\partial t}+\delta t \frac{d \mathbf{u}_{\mathbf{s}}}{d t}\right) \\
& =\frac{\partial \mathbf{u}}{\partial t}+\left(\mathbf{u}+\mathbf{u}_{\mathbf{s}}\right) \cdot \nabla \mathbf{u}+\frac{d \mathbf{u}_{\mathbf{s}}}{d t}
\end{aligned}
$$

Because we want to consider solely the component of acceleration that the larva feels due to the flow, we drop the swimming velocity derivative to obtain the flow acceleration at the larval position:

$$
\mathbf{a}=\frac{\partial \mathbf{u}}{\partial t}+\left(\mathbf{u}+\mathbf{u}_{\mathbf{s}}\right) \cdot \nabla \mathbf{u}
$$


An examination of our swimming and flow data indicates that the swimming velocity term is on average smaller than the flow acceleration term by a factor of $\sim 10$.

\subsubsection{Statistical analysis of non-acceleration turbulence fields as larval dive triggers}

The following tables document the results of the statistical analyses (modified $t$ test and Wilcoxon rank sum test) comparing the means and medians (respectively) of the distributions $P(T \mid$ larva dives $)$ and $P(T \mid$ larva does not dive $)$, for a set of mean hydromechanical parameters $T$. The normally distributed mean $e_{x x}$ and $e_{z z}$ (horizontal and vertical normal strain rates) have the means of their distributions compared in Table S1, while the nonnormally distributed fields, $\left|\omega_{y}\right|,\left|e_{x z}\right|$, and $|\alpha|$ have their distribution medians compared in Table S2. Mean hydromechanical parameters are computed over the specified temporal interval in the first columns, and results are separated by flow regime. 


\begin{tabular}{|c|c|c|c|c|c|}
\hline $\begin{array}{l}\text { Time interval } \\
\text { prior to dive } \\
\text { onset }(\mathrm{s})\end{array}$ & Field & sd & $t$ & $\begin{array}{l}95 \% \text { CI on } \\
\left|\mu_{d}-\mu_{n d}\right|\end{array}$ & $p$-value \\
\hline & & Unforced Regime & & & \\
\hline 0.33 & Horizontal & 0.06 & 1.75 & {$[-0.002,0.03]$} & 0.08 \\
\hline 0.66 & normal & 0.05 & 1.46 & {$[-0.004,0.03]$} & 0.14 \\
\hline 1.00 & strain rate & 0.06 & 1.78 & {$[-0.001,0.03]$} & 0.07 \\
\hline 1.33 & $e_{x x}$ & 0.05 & 1.68 & {$[-0.002,0.03]$} & 0.09 \\
\hline 1.66 & & 0.06 & 1.22 & {$[-0.007,0.03]$} & 0.22 \\
\hline 2.00 & & 0.05 & 2.20 & {$[0.002,0.03]$} & 0.02 \\
\hline 2.33 & & 0.06 & 1.63 & {$[-0.003,0.03]$} & 0.1 \\
\hline 2.66 & & 0.05 & 0.56 & {$[-0.01,0.02]$} & 0.57 \\
\hline 3.00 & & 0.06 & 1.75 & {$[-0.002,0.03]$} & 0.08 \\
\hline 0.33 & Vertical & 0.05 & 1.10 & {$[-0.007,0.02]$} & 0.27 \\
\hline 0.66 & normal & 0.05 & 0.84 & {$[-0.01,0.02]$} & 0.40 \\
\hline 1.00 & strain rate & 0.05 & 1.34 & {$[-0.005,0.02]$} & 0.18 \\
\hline 1.33 & $e_{z z}$ & 0.04 & 1.17 & {$[-0.006,0.02]$} & 0.24 \\
\hline 1.66 & & 0.04 & 0.20 & {$[-0.01,0.01]$} & 0.83 \\
\hline 2.00 & & 0.04 & 1.65 & {$[-0.002,0.02]$} & 0.09 \\
\hline 2.33 & & 0.04 & 1.27 & {$[-0.005,0.02]$} & 0.20 \\
\hline 2.66 & & 0.04 & 0.88 & {$[-0.007,0.01]$} & 0.37 \\
\hline \multirow[t]{2}{*}{3.00} & & 0.04 & 1.26 & {$[-0.005,0.02]$} & 0.20 \\
\hline & & Forced Regime & & & \\
\hline 0.33 & Horizontal & 0.09 & -1.33 & {$[-0.06,0.01]$} & 0.18 \\
\hline 0.66 & normal & 0.1 & -0.92 & {$[-0.05,0.2]$} & 0.35 \\
\hline 1.00 & strain rate & 0.1 & -0.99 & {$[-0.06,0.01]$} & 0.32 \\
\hline 1.33 & $e_{x x}$ & 0.1 & -1.28 & {$[-0.06,0.01]$} & 0.2 \\
\hline 1.66 & & 0.1 & -1.32 & {$[-0.06,0.01]$} & 0.18 \\
\hline 2.00 & & 0.09 & -0.80 & {$[-0.05,0.02]$} & 0.42 \\
\hline 2.33 & & 0.09 & -1.92 & {$[-0.07,0.001]$} & 0.06 \\
\hline 2.66 & & 0.09 & -1.13 & {$[-0.05,0.01]$} & 0.25 \\
\hline 3.00 & & 0.1 & -0.68 & {$[-0.05,0.02]$} & 0.49 \\
\hline 0.33 & Vertical & 0.1 & -0.89 & {$[-0.05,0.02]$} & 0.37 \\
\hline 0.66 & normal & 0.12 & 0.32 & {$[-0.03,0.05]$} & 0.78 \\
\hline 1.00 & strain rate & 0.11 & -0.33 & {$[-0.05,0.03]$} & 0.73 \\
\hline 1.33 & $e_{z z}$ & 0.11 & -1.31 & {$[-0.07,0.01]$} & 0.19 \\
\hline 1.66 & & 0.11 & -1.05 & {$[-0.06,0.02]$} & 0.29 \\
\hline 2.00 & & 0.1 & -0.4 & {$[-0.05,0.03]$} & 0.68 \\
\hline 2.33 & & 0.11 & -1.67 & {$[-0.07,0.006]$} & 0.09 \\
\hline 2.66 & & 0.1 & 0.39 & {$[-0.03,0.04]$} & 0.69 \\
\hline 3.00 & & 0.11 & -0.74 & {$[-0.05,0.02]$} & 0.45 \\
\hline
\end{tabular}

Table S1: Modified $t$-test for mean hydromechanical parameters experienced by diving versus non-diving larvae: the comparison of distributions $P(T \mid$ larva dives $)$ and $P(T \mid$ larva does not dive $)$ for mean parameter $T$, where mean is computed over stated temporal window prior to dive onset. The null hypothesis states that $\mu_{d}=\mu_{n d}$ while the alternate hypothesis states that they differ. Degrees of freedom are $d f=150$ for no turbulence, and $d f=104$ for low turbulence. Significance level is $\alpha=0.05$, with significant results in bold. 


\begin{tabular}{|c|c|c|c|c|}
\hline $\begin{array}{l}\text { Time interval } \\
\text { prior to dive } \\
\text { onset }(\mathrm{s})\end{array}$ & Field & Rank sum & $\mathrm{Z}$ & p-value \\
\hline & & Unforced Regime & & \\
\hline 0.33 & Vorticity & 5968 & 0.56 & 0.57 \\
\hline 0.66 & magnitude & 5716 & -0.35 & 0.71 \\
\hline 1.00 & $\left|\omega_{y}\right|$ & 6126 & 1.14 & 0.25 \\
\hline 1.33 & & 6024 & 0.77 & 0.44 \\
\hline 1.66 & & 5951 & 0.50 & 0.61 \\
\hline 2.00 & & 5983 & 0.62 & 0.53 \\
\hline 2.33 & & 5867 & 0.19 & 0.84 \\
\hline 2.66 & & 5799 & -0.05 & 0.95 \\
\hline 3.00 & & 5982 & 0.6 & 0.53 \\
\hline 0.33 & Shear strain & 5647 & -0.61 & 0.53 \\
\hline 0.66 & rate magnitude & 5745 & -0.25 & 0.80 \\
\hline 1.00 & $\left|e_{x z}\right|$ & 6050 & 0.86 & 0.38 \\
\hline 1.33 & & 5764 & -0.18 & 0.85 \\
\hline 1.66 & & 5825 & 0.03 & 0.96 \\
\hline 2.00 & & 5508 & -1.12 & 0.26 \\
\hline 2.33 & & 5827 & 0.04 & 0.96 \\
\hline 2.66 & & 5750 & -0.23 & 0.81 \\
\hline 3.00 & & 6025 & 0.77 & 0.43 \\
\hline 0.33 & Angular & 5415 & 1.20 & 0.23 \\
\hline 0.66 & acceleration & 5455 & 1.36 & 0.17 \\
\hline 1.00 & magnitude & 5431 & 1.27 & 0.20 \\
\hline 1.33 & $|\alpha|$ & 5438 & 1.29 & 0.19 \\
\hline 1.66 & & 5328 & 0.86 & 0.38 \\
\hline 2.00 & & 5340 & 0.90 & 0.36 \\
\hline 2.33 & & 5299 & 0.74 & 0.46 \\
\hline 2.66 & & 5188 & 0.3 & 0.76 \\
\hline \multirow[t]{2}{*}{3.00} & & 5083 & -0.10 & 0.91 \\
\hline & & Forced Regime & & \\
\hline 0.33 & Vorticity & 2940 & 0.65 & 0.51 \\
\hline 0.66 & magnitude & 3004 & 1.06 & 0.28 \\
\hline 1.00 & $\left|\omega_{y}\right|$ & 2940 & 0.65 & 0.51 \\
\hline 1.33 & & 2838 & 0.01 & 0.98 \\
\hline 1.66 & & 2954 & 0.74 & 0.45 \\
\hline 2.00 & & 2954 & 0.74 & 0.45 \\
\hline 2.33 & & 2930 & 0.59 & 0.55 \\
\hline 2.66 & & 2805 & -0.18 & 0.84 \\
\hline 3.00 & & 2917 & 0.51 & 0.60 \\
\hline 0.33 & Shear strain & 2983 & 0.92 & 0.35 \\
\hline 0.66 & rate magnitude & 3080 & 1.54 & 0.12 \\
\hline 1.00 & $\left|e_{x z}\right|$ & 2978 & 0.89 & 0.36 \\
\hline 1.33 & & 2988 & 0.96 & 0.33 \\
\hline 1.66 & & 3082 & 1.55 & 0.12 \\
\hline 2.00 & & 3189 & 2.23 & 0.02 \\
\hline 2.33 & & 2961 & 0.78 & 0.42 \\
\hline 2.66 & & 3105 & 1.69 & 0.08 \\
\hline 3.00 & & 2876 & 0.25 & 0.80 \\
\hline 0.33 & Angular & 2401 & 1.41 & 0.15 \\
\hline 0.66 & acceleration & 2299 & 0.67 & 0.49 \\
\hline 1.00 & magnitude & 2337 & 0.95 & 0.34 \\
\hline 1.33 & $|\alpha|$ & 2309 & 0.75 & 0.45 \\
\hline 1.66 & & 2352 & 1.05 & 0.28 \\
\hline 2.00 & & 2333 & 0.92 & 0.36 \\
\hline 2.33 & & 2365 & 1.15 & 0.24 \\
\hline 2.66 & & 2353 & 1.06 & 0.28 \\
\hline 3.00 & & 2305 & 0.71 & 0.47 \\
\hline
\end{tabular}

Table S2: Wilcoxon rank sum test comparing medians of mean hydromechanical parameters experienced by diving versus non-diving larvae: the comparison of distributions $P(T \mid$ larva dives and $P(T \mid$ larva does not dive) for mean parameter $T$, where mean is computed over stated temporal window prior to dive onset. The null hypothesis states that medians $M_{d}=M_{n d}$ while the alternate hypothesis states that they differ. 


\section{Chapter 5}

\section{Light stimulates swimming behavior of larval eastern oysters (Crassostrea virginica) in turbulent flow}

\subsection{Abstract}

${ }^{1}$ Planktonic larvae of the eastern oyster (Crassostrea virginica) are able to regulate their vertical position in the water, but the environmental cues responsible for this regulation, particularly in turbulent settings, remain unclear. We quantified the swimming response of late-stage oyster larvae in a grid-stirred turbulence tank to determine how light affects the swimming behavior of larvae over a range of hydrodynamic conditions similar to their natural coastal environments. We used particle image velocimetry and larval tracking to isolate larval swimming from local flow and to quantify three behavioral metrics: vertical swimming direction, probability of dives, and proportion of larvae swimming helically. We compared these metrics across turbulence levels ranging from still water $\left(\epsilon=0 \mathrm{~cm}^{2} \mathrm{~s}^{-3}\right)$ to surf zone-like conditions $\epsilon=0.4 \mathrm{~cm}^{2} \mathrm{~s}^{-3}$ ) in light and dark. In all turbulence levels, light had no effect on the proportion of upward swimming larvae, but elicited modest increases in the proportion of helically swimming and diving behaviors. We further examined the effect of light and turbulence on specific characteristics of helical trajectories, and found that these

\footnotetext{
${ }^{1}$ Currently in revision as "Wheeler JD, Luo EY, Helfrich KR, Anderson EJ, Starczak VR and Mullineaux LS. Light stimulates swimming behavior of larval eastern oysters (Crassostrea virginica) in turbulent flow", for Marine Ecology Progress Series.
} 
environmental cues induce changes to both vertical and horizontal velocities of helically swimming larvae, changing the helix geometry. The more frequent appearance of these behaviors in light is unlikely to enhance settlement success, but may serve an anti-predatory function. The rarity of dives and helical swimming in higher turbulence suggests that larval swimming responses to light may become ineffective in highly turbulent conditions.

\subsection{Introduction}

The Eastern oyster (Crassostrea virginica), like many benthic marine invertebrates, develops through a series of free-swimming planktonic larval stages of interest to both larval ecologists and fisheries managers. This native to the east coast of North America has high economic value to shellfisheries and recreational value for water clarification [128, 129, 130], but current numbers have declined to $1 \%$ of historical population due to a combination of overharvesting and environmental perturbations [131, 132]. Understanding larval behavior during planktonic stages is important for both dispersal modelling $[15,45,133]$ and effective population restoration through larval supply. For larval supply, the behavior in the competent-to-settle larval stage is of most interest, as successful larval recruitment is crucial to adult survival and reproduction [91, 134, 135]. Larval oyster recruitment in particular relies on larvae locating and settling in shallow water on rough substrate [136].

Previous studies have shown strong correlations between physical habitat and oyster larval recruitment [137, 97], suggesting that physical characteristics in the water column may mediate larval behavior. Indeed, larval oysters appear to use acoustic signatures typical of oyster reefs [30] and chemical cues released by adult oysters [95] to initiate settlement. Additionally, oyster larvae appear to respond to turbulence with a range of behaviors: larval eastern oysters have been reported to increase downward swimming [27], upward swimming [28] and diving [29] with changes in local flow conditions.

Whether light plays a role in the regulation of larval oyster swimming and settlement behavior remains unclear. Larval oysters are negatively buoyant and need to swim upwards to maintain position in the water column, in response to gravity and possibly light $[93,6]$. A response to light has been widely reported in larvae of other marine groups such as gastropods [31], crustaceans [32, 33], and ascidians [34, 35]. Additionally this response to light may also vary with larval ontogeny, as late-stage oyster pediveligers that are competent 
to settle into a benthic habitat may instead display negative phototaxis to move downward in the water column. Indeed, other marine invertebrate larvae appear to display ontogenic shifts in their response to light $[138,35]$. It is unclear if light influences settlement success and metamorphosis in larval oysters; confounding effects such as temperature and turbidity may account for contradictory results in the literature (see [6] for review).

As addressed above, most investigations of larval behavioral changes in light focus on vertical swimming direction as a positive or negative phototactic response. A less well-studied question is whether the characteristic behaviors of larvae change significantly with light; that is, do larvae exhibit diel behavioral changes? Competent larval oysters are especially useful for investigating this question, due to distinct behaviors such as helical swimming (exploratory corkscrew swimming trajectories) and diving (transient rapid downward acceleration) that can be readily observed and compared between light regimes.

Our study aims to quantify the swimming response of oyster pediveligers to light, and determine whether this response varies over a range of turbulence conditions typical of their natural coastal environment. This dual-factor approach allows us to explore turbulence thresholds of light-induced behaviors, and to evaluate whether particular larval responses might occur more commonly in day or night time conditions. We also investigate the potential utility of light as a cue to enhance settlement success in larvae. Larval behavior is quantified by observing the proportion of larvae: 1) swimming upward, 2) diving, and 3) swimming helically. Larval vertical swimming is of interest because it provides a populationlevel indicator for active settlement. The dive is an active behavior that larvae may use for either settlement or predation escape (e.g. [96, 29]), whereas helical swimming may be used in exploration or feeding (e.g. [115, 139]).

\subsection{Methods}

\subsubsection{Larval culture}

Larval eastern oysters used for this experiment were obtained from the Aquaculture Research Corporation in Dennis, Massachusetts at a size of $200-300 \mu \mathrm{m}$ and cultured as in [28]. Larvae were maintained in $3 \mu \mathrm{m}$-filtered, aerated seawater at ambient field temperature $\left(20-22^{\circ} \mathrm{C}\right)$ and salinity $(33 \mathrm{psu})$, in covered $16 \mathrm{~L}$ plastic buckets. Larvae were kept at low densities to minimize interactions $\left(<3\right.$ larvae $\left.\mathrm{mL}^{-1}\right)$ and fed daily a suspension of 
haptophyte Isochrysis sp. $\left(\sim 9 \times 10^{5}\right.$ cells $\mathrm{mL}^{-1}$ in filtered seawater $)$. Experimental trials were conducted within 2 days of larval acquisition, during which $>80 \%$ of the larvae were observed to have eyespots (a common indicator of competency [103]).

\subsubsection{Experimental setup}

The experiments were conducted in a grid-stirred turbulence tank $(44.5 \times 44.5 \times 90 \mathrm{~cm}$; described in [28]), filled with $3 \mu \mathrm{m}$-filtered seawater at $\sim 20^{\circ} \mathrm{C}$, in a temperature-controlled chamber at $\sim 20^{\circ} \mathrm{C}$. The two horizontal grids, separated vertically by $45 \mathrm{~cm}$, were constructed of $1 \times 1 \mathrm{~cm}$ acrylic bars spaced $5 \mathrm{~cm}$ apart. The grids were attached to a drive rod that oscillated them vertically in phase with an amplitude of $5 \mathrm{~cm}$ at a specified frequency. In the light treatment, our visible light source $(2700 \mathrm{~K}$, PAR of 40.93 microeinsteins at water surface) was placed on top of the tank to emulate the direction of light experienced by larvae in nature.

For each experimental trial, larvae were gently introduced into the tank at densities of $0.36-0.6 \mathrm{~mL}^{-1}$. The tank was then seeded with neutrally buoyant polystyrene particles $\left(3.0-3.4 \mu \mathrm{m}\right.$ diameter, Spherotech) to a density of $\sim 4.2 \times 10^{4}$ particles $\mathrm{mL}^{-1}$ for flow characterization by particle image velocimetry (PIV). A monochrome high-speed camera (Photron Fastcam SA3, $1024 \times 1024$ pixel resolution), was focused on a $\sim 3 \times 3 \mathrm{~cm}$ field of view in the center of the tank, equidistant from the grids. A near-infrared laser (Oxford Lasers, Firefly $300 \mathrm{~W}, 1000 \mathrm{~Hz}, 808 \mathrm{~nm}$ ), oriented perpendicularly to the camera, illuminated the field of view with a laser sheet approximately $1 \mathrm{~mm}$ thick, which was unaffected by the presence or absence of visible light.

The larvae were subjected to either dark or light conditions under 5 turbulence levels, ranging from unforced flow $\left(\epsilon=0 \mathrm{~cm}^{2} \mathrm{~s}^{-3}\right)$ and low turbulence $\left(\epsilon=0.002 \mathrm{~cm}^{2} \mathrm{~s}^{-3}\right)$ to conditions similar to coastal surf zones $\left(\epsilon=0.4 \mathrm{~cm}^{2} \mathrm{~s}^{-3}\right)$, with energy dissipation rates estimated as in [28]. After larvae and particles were introduced, the tank was permitted a 20-minute relaxation period, with the still water (unforced) treatment conducted after this period. Sets of $45 \mathrm{~s}$ video sequences, recorded at 60 frames per second, were collected for each turbulence level as described in [28]. Four replicate trials for each light condition, each with a separate batch of larvae, were conducted by cycling through all 5 turbulence levels. The turbulence levels were sequenced in a different order, in a Latin square configuration post-unforced flow, in each trial (Table 5-1) to reduce possible confounding temporal effects. 


\begin{tabular}{|l|l|l|l|}
\hline Treatment & $\begin{array}{l}\text { Turbulence level } \\
\left(\mathrm{cm}^{2} \mathrm{~s}^{-3}\right)\end{array}$ & $\begin{array}{l}\text { Number of } \\
45 \text {-second datasets }\end{array}$ & $\begin{array}{l}\text { Number of } \\
\text { larvae tracked }\end{array}$ \\
\hline dark & 0 & 5 & 168 \\
\hline dark & 0.027 & 4 & 512 \\
\hline dark & 0.373 & 3 & 368 \\
\hline dark & 0.002 & 4 & 207 \\
\hline dark & 0.064 & 3 & 390 \\
\hline light & 0 & 5 & 307 \\
\hline light & 0.027 & 4 & 465 \\
\hline light & 0.373 & 3 & 737 \\
\hline light & 0.002 & 4 & 230 \\
\hline light & 0.064 & 3 & 487 \\
\hline
\end{tabular}

Table 5-1: An example of experimental design for Trial 4. Light treatments were randomized and turbulence orders were assigned by Latin square in an attempt to minimize the effects of time. Consult Supplemental Materials for complete experimental design of Trials 1-4.

\subsubsection{Local flow subtraction to isolate larval swimming velocities}

Larval swimming velocity was calculated for each individual by subtracting local flow velocity from the larval motion at each step in the recorded larval trajectory, fully detailed in [28]. Larval centroid positions were identified in each frame using custom LabVIEW (National Instruments) software with user-specified tolerances on larval size and pixel intensity. Larvae were tracked from frame to frame using a custom MATLAB script with a maximum search radius in subsequent frames, and frame-to-frame instantaneous velocities were thereby calculated. Individual mean larval velocities were computed by averaging instantaneous velocities over the observed larval trajectory. Flow fields were estimated using PIV with DaVis v.7.2 (LaVision) software to a spatial resolution of $\sim 0.04 \mathrm{~cm}$ and velocity vector fields were imported into MATLAB. We identified annuli (inner radius $\sim 0.04$ and outer radius $\sim 0.2 \mathrm{~cm}$ ) of flow vectors around each larva and subtracted the mean annulus flow velocity from observed larval velocity, to isolate individual larval swimming velocities. Individual instantaneous larval swimming velocity time series were then used to compute the proportion of upward swimming larvae. A larva was classified as upward swimming if its mean vertical swimming velocity was positive. 

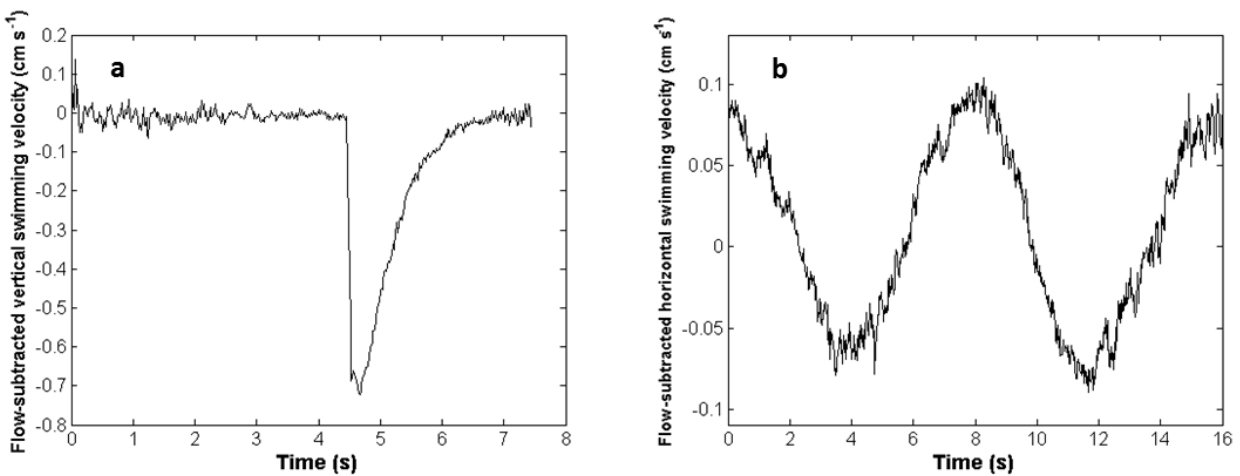

Figure 5-1: Distinct larval swimming behaviors observed in flow subtracted larval swimming velocity time series at 60 frames per second. (a) Example of a dive, as characterized by a sudden drop in vertical velocities to at least $-0.4 \mathrm{~cm} \mathrm{~s}^{-1}$. Note sharp decrease in vertical swimming velocities to under $-0.7 \mathrm{~cm} \mathrm{~s}^{-2}$, around $4.5 \mathrm{~s}$, characterizing the dive. (b) Example of a helically swimming larva characterized by a sinusoid-like shape in horizontal velocity having a period of approximately $8 \mathrm{~s}$.

\subsubsection{Identification of dives}

The dive response is a distinct behavior characterized by a rapid downward burst in speed. Dives were identified using larval instantaneous vertical swimming velocity and acceleration time-series, where acceleration was computed from the velocity time-series data using a central difference scheme. A dive was characterized by a sudden (within 1/30 s) drop in vertical velocity to values of -0.4 to $-0.8 \mathrm{~cm} \mathrm{~s}^{-1}$. A dive typically lasted approximately one second, during which time the larva slowed its descent and eventually reached near-zero vertical velocity (Fig. 5-1a). Larval trajectories were classified as dives if they reached instantaneous accelerations of $3.0 \mathrm{~cm} \mathrm{~s}^{-2}$ (150 body lengths $\mathrm{s}^{-2}$ ) for more than one time step $(1 / 60 \mathrm{~s})$, and achieved instantaneous negative vertical velocities of at least $-0.4 \mathrm{~cm} \mathrm{~s}^{-1}$ $[28]$. 


\subsubsection{Identification of helical swimming}

The corkscrew shaped path of helically swimming larvae results in a near-sinusoidal curve in horizontal velocities versus time. We searched for occasions of helical swimming by detecting sinusoidal-like motion in time-series of larval horizontal swimming velocities. A larva was categorized as helically swimming if it contained at least one full period of sinusoidal-shaped horizontal velocity (determined by visual inspection), with a minimum velocity of $0.05 \mathrm{~cm}$ $\mathrm{s}^{-1}$ in both horizontal directions (Fig. 5-1b).

\subsubsection{Analysis of behavioral data}

The effects of light and turbulence on proportion of upward swimming larvae were analyzed in the unforced regime and turbulence using two separate general linear models. The purpose of the analysis was to detect effects of light and turbulence, as well as their interaction, on vertical swimming, but we also incorporated other potential drivers of larval behavior, including larval age and time spent in the tank. The model for the unforced regime data was

$$
Y=\mu+\text { light }+ \text { trial }(\text { light })+\text { video seq. }+ \text { light } \times \text { video seq. }+ \text { error. }
$$

and the model for the turbulence data was

$$
\begin{aligned}
Y & =\mu+\text { light }+ \text { trial }(\text { light })+\text { time }+ \text { time } \times \text { light }+ \text { turbulence level } \\
& + \text { turbulence level } \times \text { light }+ \text { error }
\end{aligned}
$$

For the turbulence data, "time" is the variable controlling the turbulence treatment order (that is, each turbulence level occurred at a different time within each trial, as the turbulence levels were reordered for each new trial). Light (2 levels), trial (4 levels), video sequence (4 levels), and time (4 levels) were categorical variables, and light was tested between trials in the light and dark treatments. Other effects were fixed and tested with the mean squares error of the ANOVA within the light and dark treatments individually. Data were separated into unforced and turbulence analyses because the unforced observations were taken prior to any turbulence observations in all trials, and the turbulence treatments between the trials were amenable to a Latin squares analysis.

The proportion of diving larvae in turbulence was also analyzed using the turbulence 
general linear model. The proportion of helically swimming larvae was tested using a modified analysis, as helical swimming was only identified in the unforced and lowest forcing regime. This is due to the inherent challenge of identifying a multi-second behavioral pattern (a full helical period) when larvae are rapidly advected through the field of view in more highly turbulent flow. For helix data, analysis on each variable was done with a split plots design with light as the main factor, trials nested within light and turbulence as the subplot factor. In addition to the proportion of helically swimming larvae, we also applied this model to relevant characteristics of helix geometry: translational velocity (mean vertical swimming speed during an identified half helix) and speed of helical swimming (mean horizontal swimming speed throughout a half helix).

\subsection{Results}

\subsubsection{Vertical swimming}

In both light and dark, larvae generally swam downward in the unforced flow regime, upward in moderate turbulence, and displayed decreased upward swimming in high turbulence (Fig. 5 -2). In the unforced flow regime, only the video sequence number (time spent in the tank) had a significant effect on the proportion of upward swimmers, with light, trial, and the interactive effect of light and video sequence showing no significance (Table 5-2). This suggests that larval behavior may have changed over time spent in the tank (within a trial) with either acclimation or fatigue. In the turbulence regimes, light had no significant effect on upward swimming, either by itself, or in interaction with time or turbulence level, which suggests that larvae did not respond phototactically. Turbulence level, time, and trial all had a significant effect on upward swimming (Table 5-3): the unforced and highly forced turbulence regimes $\left(\epsilon=0 \mathrm{~cm}^{2} \mathrm{~s}^{-3}\right.$ and $0.373 \mathrm{~cm}^{2} \mathrm{~s}^{-3}$ respectively) had a lower proportion of upward swimming larvae than the moderate turbulence regimes $\left(\epsilon=0.002-0.064 \mathrm{~cm}^{2}\right.$ $\mathrm{s}^{-3}$ ). Both the time (within the trial) and the trial impacted upward swimming, suggesting acclimation to the tank or fatigue within a trial, and potentially age effects since trials took place over the entire larval competency period. Larval vertical swimming was thus mediated strongly by turbulent flow conditions and larval age, but not at all by light. 


\begin{tabular}{|l|l|l|l|l|l|}
\hline Source & Type III SS & $d f$ & MS & $F$-ratio & $p$-value \\
\hline Light & 0.02 & 1 & 0.02 & 0.95 & 0.36 \\
Video sequence & 0.29 & 3 & 0.1 & 3.2 & $\mathbf{0 . 0 5}$ \\
Light $\times$ Video Sequence & 0.03 & 3 & 0.009 & 0.31 & 0.81 \\
Trial(light) & 0.12 & 6 & 0.02 & 0.72 & 0.63 \\
Error & 0.53 & 18 & 0.03 & & \\
\hline
\end{tabular}

Table 5-2: Results of ANOVA on general linear model for proportion of upward swimming larvae in unforced flow, testing for effects of light, video sequence number (acclimation/fatigue), and trial (aging). Significant results are bolded, with a significance level $\alpha=0.05$.

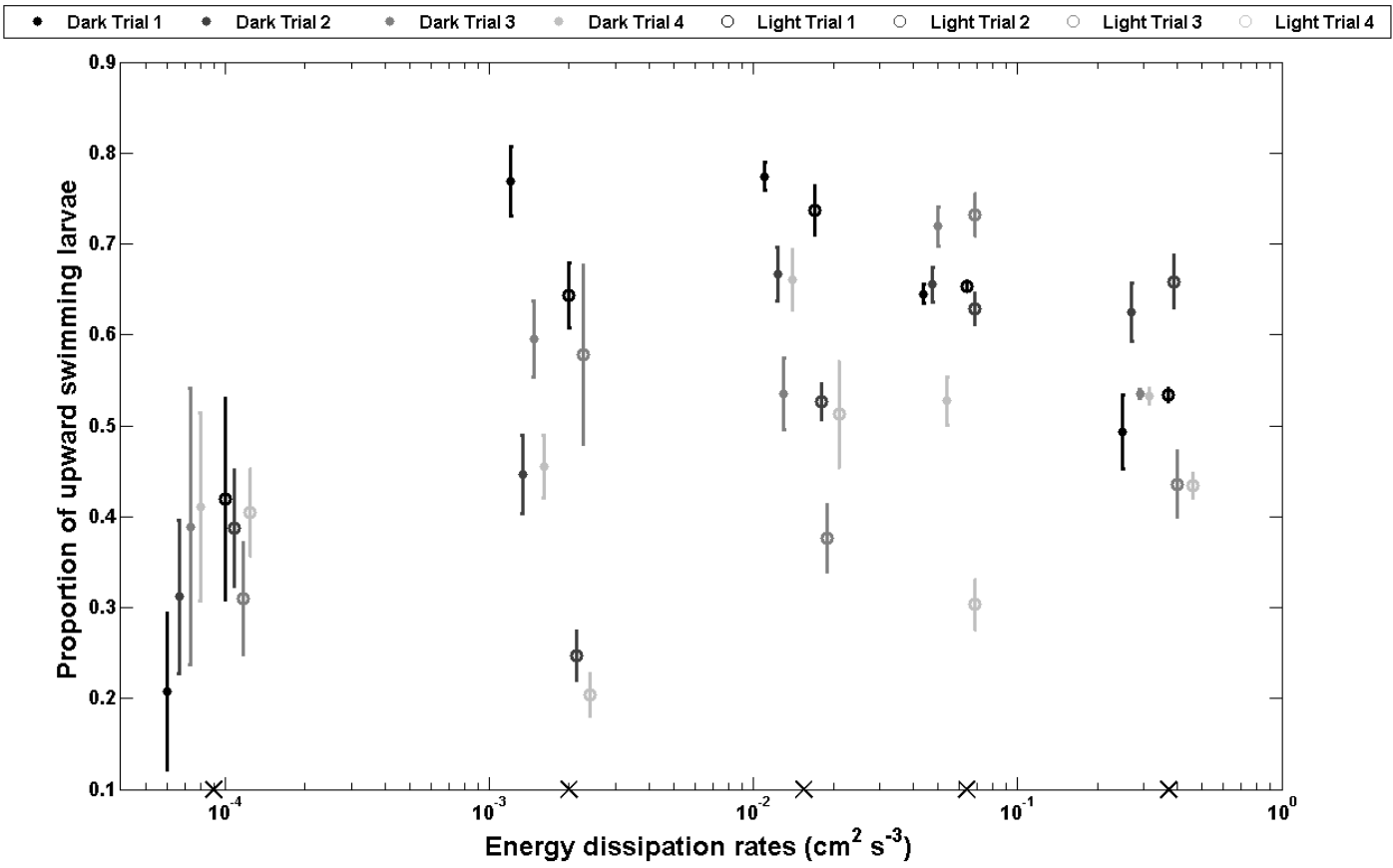

Figure 5-2: Mean ( $\pm \mathrm{SE}$ ) proportion of upward swimming larvae for each trial vs. turbulence level (energy dissipation rate), in dark (closed circles) and light (open circles), with trials 1-4 denoted for each by decreasing color intensity. Means are grouped by dark and light and are artificially spaced for clarity: black x symbols on the energy dissipation rate axis denote values at which measurements were taken. Larvae displayed primarily downward swimming in unforced flow regime, upward swimming in moderate turbulence, and decreased upward swimming in high turbulence. Upward swimming was less common in later trials than earlier trials in both light and dark.

\subsubsection{Probability of dives}

We observed 367 dives in total, predominantly in the unforced and low turbulence regimes. The mean proportion of dives differed significantly between turbulence treatments (Table 


\begin{tabular}{|l|l|l|l|l|l|}
\hline Source & Type III SS & $d f$ & MS & $F$-ratio & $p$-value \\
\hline Light & 0.06 & 1 & 0.06 & 2 & 0.21 \\
Time & 0.26 & 3 & 0.08 & 43.59 & $<\mathbf{0 . 0 0 1}$ \\
Turbulence level & 0.07 & 3 & 0.02 & 12.35 & $<\mathbf{0 . 0 0 1}$ \\
Time $\times$ Light & 0.01 & 3 & 0.004 & 2.05 & 0.16 \\
Turbulence level $\times$ Light & 0.02 & 3 & 0.006 & 3.02 & 0.07 \\
Trial (light) & 0.19 & 6 & 0.03 & 15.94 & $<\mathbf{0 . 0 0 1}$ \\
Error & 0.02 & 12 & 0.002 & & \\
\hline
\end{tabular}

Table 5-3: Results of ANOVA on general linear model for proportion of upward swimming larvae in forced flow, testing for effects of light, time (turbulence treatment order), turbulence level, and trial (aging). Significant results are bolded, with a significance level $\alpha=0.05$.

$5-4)$, where the proportion of dives was highest in the lowest turbulence treatment and decreased with increasing turbulence (Fig. 5-3). There was no significant interaction effect of turbulence and light, and light alone had a borderline significant effect on dive proportion (Table 5-4). The difficulty in ascertaining a light response may be due to the low power of the test and a significant variability among trials. Moreover, there was a significant interaction between light and the time that the turbulence treatment was administered (Table 5-4). In the fourth treatment administered, for instance, the proportion of diving larvae was higher in light than in dark (Tukey's HSD test; $p=0.006$ ).

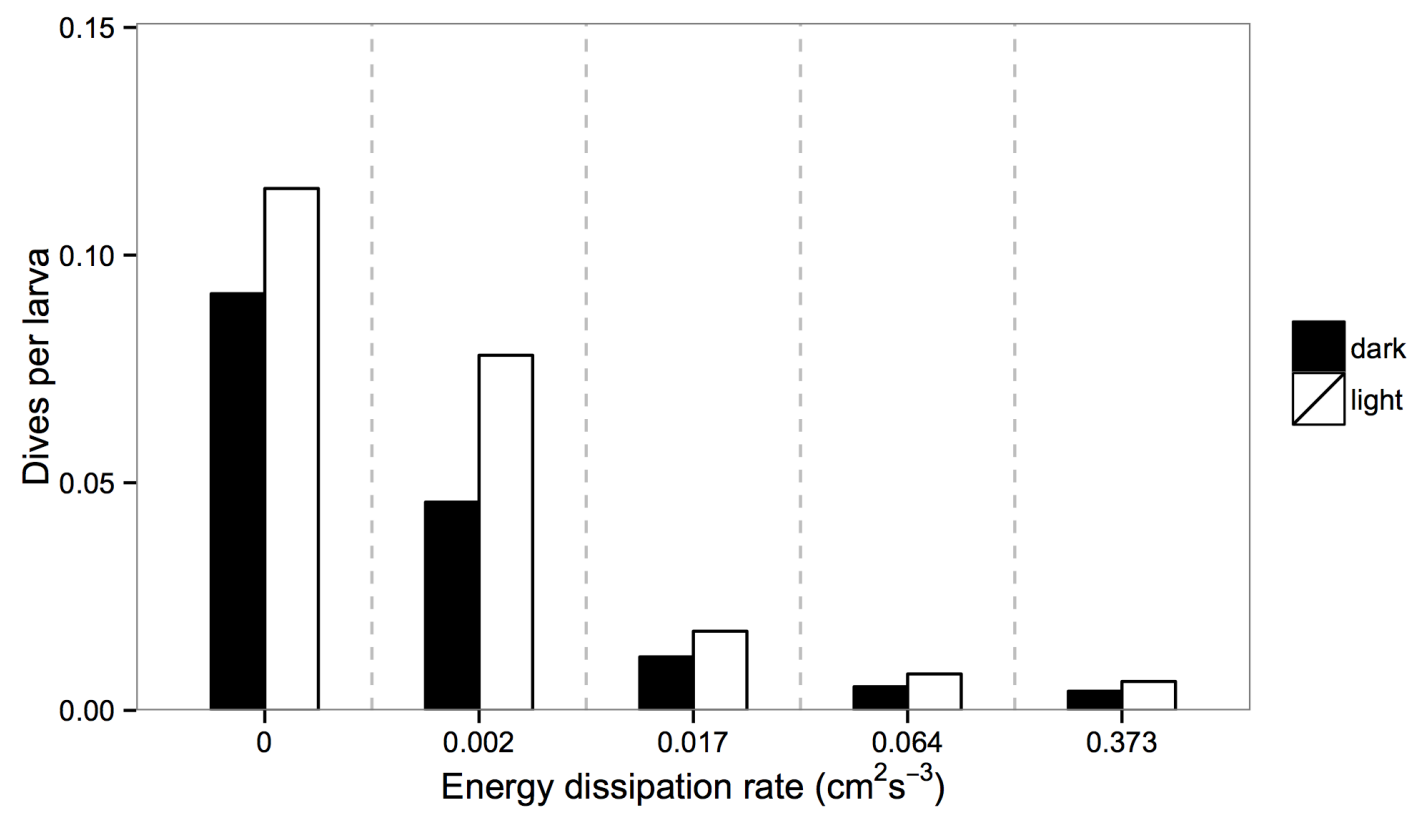

Figure 5-3: Probability of dives with respect to turbulence, as characterized by energy dissipation rate, in dark (black bars) and light (white bars). 


\begin{tabular}{|l|l|l|l|l|l|}
\hline Source & Type III SS & $d f$ & MS & $F$-ratio & $p$-value \\
\hline Light & 0.01 & 1 & 0.01 & 4.32 & 0.08 \\
Time & 0.003 & 3 & 0.001 & 1.27 & 0.32 \\
Turbulence level & 0.14 & 3 & 0.04 & 50.34 & $<\mathbf{0 . 0 0 1}$ \\
Time $\times$ Light & 0.01 & 3 & 0.003 & 3.99 & $\mathbf{0 . 0 3}$ \\
Turbulence level $\times$ Light & 0.002 & 3 & 0.0009 & 0.94 & 0.44 \\
Trial (light) & 0.02 & 6 & 0.003 & 3.53 & $\mathbf{0 . 0 3}$ \\
Error & 0.01 & 12 & 0.0009 & & \\
\hline
\end{tabular}

Table 5-4: Results of ANOVA on general linear model for proportion of diving larvae in forced flow, testing for effects of light, time (turbulence treatment order), turbulence level, and trial (aging). Significant results are bolded, with a significance level $\alpha=0.05$.

\subsubsection{Helical swimming}

Exposure of larvae to light did not effect the proportion of helically swimming larvae, but this proportion was significantly lower in the low forcing regime than in the unforced regime. The interactive effect of light and turbulence was non-significant, as was the effect of trial (Table 5-5).

Mean helical swimming speed was higher in larvae exposed to light, with borderline significance. Turbulence had no significant effect on helical speed, nor did the interaction between turbulence and light (Table 5-6).

Mean vertical swimming speed of helically swimming larvae did not differ significantly between light and dark treatments. The interaction between light and turbulence was significant, however, as was the turbulence main effect (Table 5-7). Paired comparison of the light and turbulence treatment means (Tukey's HSD test) revealed that in the dark the mean vertical speed was lower in unforced turbulence treatment than in the low forcing turbulence treatment $(p=0.013)$. The dark unforced treatment also had lower mean vertical swimming speed than the light forced turbulence treatment $(p=0.0232)$. No other pairs of means differed.

\subsection{Discussion}

We found that light induced modest increases in dives and modified the geometry of larval helical trajectories, but that turbulence had a much stronger effect on these behaviors. Light had no effect on the proportion of upward swimming larvae (i.e. population-level behavior) at any turbulence level. In contrast, both turbulence level and conditions relating to larval 


\begin{tabular}{|l|l|l|l|l|l|}
\hline Source & Type III SS & $d f$ & MS & $F$-ratio & $p$-value \\
\hline Light & 0.004 & 1 & 0.004 & 2.01 & 0.20 \\
Turbulence Level & 0.006 & 1 & 0.006 & 10.68 & $\mathbf{0 . 0 2}$ \\
Turbulence Level $\times$ Light & 0.0006 & 1 & 0.0006 & 0.96 & 0.36 \\
Trial(light) & 0.01 & 6 & 0.002 & 3.22 & 0.09 \\
Error & 0.003 & 6 & 0.0006 & & \\
\hline
\end{tabular}

Table 5-5: Results of ANOVA on split plots design for proportion of helically swimming larvae in unforced and low forcing flow, testing for effects of light, turbulence level, and trial (aging). Significant results are bolded, with a significance level $\alpha=0.05$.

\begin{tabular}{|l|l|l|l|l|l|}
\hline Source & Type III SS & $d f$ & MS & $F$-ratio & $p$-value \\
\hline Light & 0.01 & 1 & 0.01 & 5.02 & 0.06 \\
Turbulence Level & 0.00001 & 1 & 0.00001 & 0.005 & 0.94 \\
Turbulence Level $\times$ Light & 0.0002 & 1 & 0.0002 & 0.068 & 0.80 \\
Trial(light) & 0.01 & 6 & 0.003 & 1.03 & 0.48 \\
Error & 0.02 & 6 & 0.003 & & \\
\hline
\end{tabular}

Table 5-6: Results of ANOVA on split plots design for mean helix swimming speed in unforced and low forcing flow, testing for effects of light, turbulence level, and trial (aging). Significant results are bolded, with a significance level $\alpha=0.05$.

\begin{tabular}{|l|l|l|l|l|l|}
\hline Source & Type III SS & $d f$ & MS & $F$-ratio & $p$-value \\
\hline Light & 0.002 & 1 & 0.002 & 0.83 & 0.39 \\
Turbulence Level & 0.01 & 1 & 0.01 & 16.4 & $\mathbf{0 . 0 0 6}$ \\
Turbulence Level $\times$ Light & 0.005 & 1 & 0.005 & 6.72 & $\mathbf{0 . 0 4}$ \\
Trial(light) & 0.02 & 6 & 0.002 & 3.98 & 0.06 \\
Error & 0.004 & 6 & 0.0007 & & \\
\hline
\end{tabular}

Table 5-7: Results of ANOVA on split plots design for mean helix vertical swimming speed in unforced and low forcing flow, testing for effects of light, turbulence level, and trial (aging). Significant results are bolded, with a significance level $\alpha=0.05$. 
fatigue and age had strong impacts on larval swimming direction.

These effects of light, modest as they are, have several implications for larval swimming behavior. Diving is an active downward acceleration that may be attributed to a settlement response $[27,28,29]$. It is possible that late-stage oyster larvae display ontogenic shifts in larval phototaxis through increased dive frequencies in light, however, fast, downward propulsion may also allow them to escape from predation. Indeed, oyster larvae exhibit higher dive probabilities in response to the fluid acceleration they experience [29], which may be characteristic of an approaching predator [112,113]. Similarly, helical swimming may also act as an antipredatory response while engaging in exploratory swimming, as helical swimming clears large foraging volumes with a minimal hydromechanical presence to predators [139]. If dives and helical swimming serve as effective antipredatory responses, they may occur more readily in light if predators use visual cues to detect prey [140]. Alternatively, helical swimming may increase the precision of navigation during phototaxis, as demonstrated in simulations of annelid swimming [141, 142]. While no phototactic response is obvious in the proportion of upward swimming larvae in our study, the change in helical swimming characteristics in light demonstrates a behavior benefitting phototactic larvae. Such results indicate the importance of considering multiple swimming metrics when quantifying a behavioral response.

The change in helix speed and translational velocity in response to light suggests that larval oysters have active control over helical swimming behavior. Furthermore, helical swimming persists in turbulence, suggesting a robust larval control of swimming behaviors. Such control does not appear to be dictated by morphology alone [143], as larval oysters display flexibility in their helix translational and angular velocity in response to environmental cues. The increase in probability of dives in response to light was less apparent, and not significant, at higher turbulence level. Wheeler et al. [29] also observed a decrease in dive probability at higher turbulence, and suggested that although larvae dive in response to local hydrodynamic triggers, they may stop reacting to dive triggers past a threshold in higher turbulence levels. Alternatively, the rapid fluctuations in flow regimes in high turbulence may be inadequate for creating sustained dive triggers.

Light had no effect on the proportion of upward swimming larvae, which was surprising because we had expected to see some phototactic response. Despite the prevalence of positive phototaxis in larvae [1] and observations of positive phototaxis in younger oyster larvae [6], 
light had no observable effect on vertical swimming direction of our late-stage oyster larvae, suggesting an ontogenic shift in phototaxis. Ontogenic changes in phototaxis have been widely documented in larvae of eels [144], polychaetes [138, 145], crabs [146], mussels [26], nudibranchs [147], conch [148], and both larval and juvenile sole [149]. Competent larvae may cease to display positive phototaxic behavior because they no longer need to stay afloat in the water column. Further studies comparing phototactic behaviors of oyster larvae at various stages of development would be required to identify such an ontogenetic shift. A caveat to consider from our analyses, however, is the strong effect of time on larval swimming. Larvae exhibited fatigue or acclimation on short time scales and behavioral shifts on longer time scales. This uncontrolled variability may have made an effect of light on upward swimming behavior difficult to detect.

Our study further strengthens the body of evidence documenting upward swimming of larval oysters in moderate turbulence [28], which suggests that turbulence alone does not act as a cue for larval settlement. Similarly, we observed no change in the proportion of larvae which swam upward in response to light; the absence of an obvious negative phototactic response suggests that neither light alone, nor light in conjunction with turbulence, is an effective inducer of larval settlement. Nevertheless, the observed changes in larval diving and helical swimming in the presence of light suggests that they modify potentially exploratory and anti-predatory behaviors in light versus darkness. So while light does not modify larval vertical swimming direction on a population level (indicative of an active settlement response), it does indeed induce behavioral changes, suggestive of a diel behavioral shift.

\subsection{Acknowledgements}

We thank Susan Mills and Anthony Ritchie for experimental assistance, and Samuel Laney for light measurements. We gratefully acknowledge the Aquaculture Research Corporation (Dennis, MA, USA), who provided the larvae used in this study. Funding was provided by NSF grant OCE-0850419, NOAA Sea Grant NA14OAR4170074, grants from WHOI Coastal Ocean Institute, discretionary WHOI funds, a WHOI Ocean Life Fellowship to LM, a Grove City College Swezey Fund Grant to EA, and a WHOI Summer Student Fellowship to EL. 


\subsection{Supplemental Material}

Experimental design: treatment and turbulence orders were designed in an attempt to minimize the effects of time.

\begin{tabular}{|c|c|c|c|c|}
\hline Trial & Treatment & $\begin{array}{l}\text { Turbulence level } \\
\left(\mathrm{cm}^{2} \mathrm{~s}^{-3}\right)\end{array}$ & $\begin{array}{l}\text { Number of } \\
45 \text {-second datasets }\end{array}$ & $\begin{array}{l}\text { Number of } \\
\text { larvae tracked }\end{array}$ \\
\hline 1 & dark & 0 & 5 & 69 \\
\hline 1 & dark & 0.002 & 4 & 133 \\
\hline 1 & dark & 0.027 & 4 & 464 \\
\hline 1 & dark & 0.064 & 3 & 409 \\
\hline 1 & dark & 0.373 & 3 & 379 \\
\hline 1 & light & 0 & 5 & 91 \\
\hline 1 & light & 0.002 & 4 & 191 \\
\hline 1 & light & 0.027 & 4 & 450 \\
\hline 1 & light & 0.064 & 3 & 566 \\
\hline 1 & light & 0.373 & 3 & 510 \\
\hline 2 & light & 0 & 5 & 298 \\
\hline 2 & light & 0.373 & 3 & 896 \\
\hline 2 & light & 0.064 & 3 & 967 \\
\hline 2 & light & 0.027 & 4 & 767 \\
\hline 2 & light & 0.002 & 4 & 323 \\
\hline 2 & dark & 0 & 5 & 91 \\
\hline 2 & dark & 0.373 & 3 & 376 \\
\hline 2 & dark & 0.064 & 3 & 330 \\
\hline 2 & dark & 0.027 & 4 & 424 \\
\hline 2 & dark & 0.002 & 4 & 237 \\
\hline 3 & light & 0 & 5 & 220 \\
\hline 3 & light & 0.064 & 3 & 604 \\
\hline 3 & light & 0.002 & 4 & 215 \\
\hline 3 & light & 0.373 & 3 & 343 \\
\hline 3 & light & 0.027 & 4 & 382 \\
\hline 3 & dark & 0 & 5 & 68 \\
\hline 3 & dark & 0.064 & 3 & 430 \\
\hline 3 & dark & 0.002 & 4 & 210 \\
\hline 3 & dark & 0.373 & 3 & 301 \\
\hline 3 & dark & 0.027 & 4 & 398 \\
\hline 4 & dark & 0 & 5 & 168 \\
\hline 4 & dark & 0.027 & 4 & 512 \\
\hline 4 & dark & 0.373 & 3 & 368 \\
\hline 4 & dark & 0.002 & 4 & 207 \\
\hline 4 & dark & 0.064 & 3 & 390 \\
\hline 4 & light & 0 & 5 & 307 \\
\hline 4 & light & 0.027 & 4 & 465 \\
\hline 4 & light & 0.373 & 3 & 737 \\
\hline 4 & light & 0.002 & 4 & 230 \\
\hline 4 & light & 0.064 & 3 & 487 \\
\hline
\end{tabular}




\section{Chapter 6}

\section{Larval oyster swimming responses to adult chemical exudates: evidence for the desperate larva hypothesis?}

\subsection{Abstract}

Competent-to-settle larvae of benthic invertebrates can detect and respond to chemical cues originating from the benthos signaling the presence of suitable settlement sites, often in the form of conspecific adults or suitable prey species. Larvae in early stages of competency often demonstrate selectivity in responding to potential settlement cues, but this selectivity often decreases with larval age. The "desperate larva" hypothesis, originally proposed for lecithotrophic larvae, suggests that this decreased selectivity is due to dwindling energy reserves over time, and results in old larvae responding to sub-standard settlement cues. In this study, we exposed the competent, well-fed larvae of the eastern oyster (Crassostrea virginica) to two seawater treatments and observed their swimming behavior over the entire competency period. One treatment was filtered seawater containing no settlement cue, and the other was filtered seawater conditioned with adult oysters, a strong inducer for larval oyster settlement. We found that over time ( 40-60 hours post-competency) larvae exposed to no cue began behaving more like bathwater-exposed larvae, with average vertical position and vertical swimming velocity in the two treatments converging as larvae aged. Our results support an expanded version of the desperate larva hypothesis, where non-starving 
planktotrophic larvae ultimately settle due to an inability to maintain a competent larval state.

\subsection{Introduction}

The transport of larvae to the seafloor is a crucial component of a benthic invertebrate life cycle, as it represents the transition from the planktonic larval stages to adult life on the seafloor. Most studies examining this transitory period focus on larval behavior near and on the substratum (reviewed in e.g., [23, 24, 4]), where attachment and metamorphosis takes place. Larval behavior higher in the water column is less well-studied, however, and it remains unclear to what extent larvae can actively govern their downward motion when competent to settle $[25,94,27,28,29,48,98]$. Water column cues, which may change larval behavior by inducing active swimming toward or away from the benthos, are therefore important for our understanding of the settlement period. Of specific interest are chemical cues originating from the benthos, produced by prey species, conspecific adults, or corresponding biofilms [150, 151, 4].

The larvae of the eastern oyster (Crassostrea virginica) are widely used for chemical cue studies due to their documented responses to a variety of chemical cues in a laboratory setting. Gregarious settlement by larvae has been induced in both still-water and flume flow experiments. Crisp [152] was the first to demonstrate that larvae avoided settlement on bleached oyster shells, preferring shells which retained a biofilm. In still-water experiments, Tamburri et al. [40] demonstrated that the shell presence was in fact unnecessary: they observed a similar settlement response in larvae exposed to "oyster bathwater", a homogeneous solution of filtered seawater exposed to either adult oysters (without biofilms) or shell biofilms alone. In a later experiment, the peptide glycyl-glycyl-L-arginine (GGR) was found to induce larval attachment to the substrate with an almost identical dose-response curve to the conditioned seawater in homogeneous solutions [153]. Larvae exposed to both homogeneous adult conditioned seawater and GGR solutions in a flume flow were found to settle out of the flow for a range of mean flow velocities, significantly moreso than larvae which were not exposed to a chemical cue [95]. In addition to adult conditioned seawater and GGR, increased oyster larval settlement has been observed in response to bacterial supernatants [154] and ammonia [155]. With respect to habitat selection, oyster larvae will settle on whelk 
shell restoration reefs and other non-oyster sites in the field [97], indicating that settlement can occur without chemical cues put forth by adult oysters. However, field evidence suggests that chemical signaling does play a role in settlement patterns, as larvae will preferentially settle on living oyster reefs instead of broken oyster shells [135, 156, 97]. Further, heavily biofouled tiles are preferred to non-fouled tiles [157], recalling larval settlement responses to both cleaned adult oysters and their biofilms [40] and bacterial supernatants [154].

As larvae age and reach competency, they develop new behavioral responses which may facilitate settlement into suitable benthic habitats (e.g., [3]). Oyster larvae experience and respond to a variety of cues, including light [6] turbulence [27, 28, 29], and sound [30], in addition to the previously described chemical inducers. Larval responses to settlement cues may also change over the competency period, a phenomenon which has long interested larval ecologists. The desperate larva hypothesis was first proposed over sixty years ago $[158,111,159]$, and states that lecithotrophic larvae would respond to sub-standard settlement cues as they aged and their energy supplies dwindled. Since then, the hypothesis has expanded to include some planktotrophs [39], with the specification that planktotrophs, unlike lecithotrophs, become desperate to settle because of a reduced capacity to maintain the competent larval swimming state (as opposed to due to a decrease in maternal resources) [160]. Both cases are equivalent from an ecological perspective, involving delayed metamorphosis and eventual acceptance of sub-standard settlement cues, and require that larvae can persist in swimming and searching behaviors in the absence of suitable settlement cues [160]. Further, the interpretation of the hypothesis has been extended to older larvae settling in the presence of settlement inhibitors [161], no cue at all [160], or a wider range of cues [162], none of which induce settlement in younger, more selective larvae. Finally, body size [163], plankton mortality [164], and prior exposure to settlement cues [39, 165] appear to be factors influencing changes in larval responses to cues with age.

The range of cues to which oyster larvae respond, in addition to their relatively long $(>24$ hours) competency period make them a model species for careful study during competency, in order to understand how their behavior changes during the transitory period between the water column and the seafloor. Specifically, we seek to understand how cue selectivity changes with age as larvae become "desperate" to settle. In this study, we investigate how larval oyster swimming behavior changes over their competency period in still water, when exposed to one of two seawater treatments: filtered seawater (no settlement cue), and adult 
oyster conditioned bathwater (a chemical settlement cue). We expect that cue exposed larvae will swim downwards and aggregate near the bottom of the experimental tank (suggestive of settlement), while cue-less larvae will engage in exploratory swimming behavior throughout the tank and maintain a higher position in the water column. We also track larval body size, as larvae may continue to grow throughout competency [6] and their size can impact their swimming capabilities [93].

If oyster larvae become less selective to settlement cues with age, one of two outcomes is possible. If the chemical cue effect is not initially strong, larvae could become more sensitive to the chemical cue over time. In this case, larvae in the two seawater treatments would initially behave similarly and diverge in behavior over time, with the cue larvae displaying increased settlement-like behavior with age. Alternatively, if the chemical cue is a strong settlement inducer even to newly competent larvae, then the cue larvae would display settlement-like behavior irrespective of age. In this case, decreased cue selectivity would be observed if cue and cue-less larvae initially behave differently and their behavior converges over time; that is, cue-less larvae would ultimately display settlement-like behavior even in the absence of a settlement cue. If swimming behavior changes as oyster larvae age through competency, it would offer evidence for the desperate larva hypothesis in a planktotrophic species which responds to a range of settlement cues, which to our knowledge has not previously been observed.

\subsection{Methods}

\subsubsection{Study species and handling practices}

The eastern oyster, Crassostrea virginica, is a shallow water mollusc native to the North Atlantic. Adults commonly form reefs in the intertidal and sub-tidal zones and are broadcast spawners, producing planktotrophic, free-swimming larvae with a pelagic duration of 2-3 weeks $[101,6]$. The penultimate and final larval stages are the veliger and pediveliger stages, respectively. Larvae in both stages are characterized by a ciliated, retractable velum extending from a calcareous shell, which they use to swim and feed. Larvae become pediveligers upon the development of a foot and pronounced eyespot, at which point they are competent to settle out of the plankton and metamorphose [103].

We obtained larvae from two separate spawns for use in two experiments in August 
2015. The first spawn (Spawn 1) was obtained from the Martha's Vineyard Shellfish Group (Martha's Vineyard, MA, USA) and the second (Spawn 2) from the Aquaculture Research Corporation (Dennis, MA, USA). Both spawns were obtained when the larvae were mature veligers, and maintained prior to experiments in identical culture conditions: $1 \mu \mathrm{m}$-filtered, aerated seawater at ambient field temperature $\left(20-22^{\circ} \mathrm{C}\right)$ and salinity (33 psu), in covered $16 \mathrm{~L}$ plastic buckets. Larvae were kept at low densities to minimize interactions $(<3$ larvae $\left.\mathrm{mL}^{-1}\right)$ and fed daily a suspension of haptophyte Isochrysis sp. $\left(\sim 9 \times 10^{5}\right.$ cells $\mathrm{mL}^{-1}$ in filtered seawater). Larvae were maintained in these conditions $(\sim 2-3$ days $)$ until $>50 \%$ of larvae exhibited eyespots (an indicator of competency to settle), as determined by microscopic examination of a random sample of larvae. Immediately post-observation, larvae were retained from the sampling flask on a $100 \mu \mathrm{m}$ mesh filter and subsequently preserved in a solution of $95 \%$ ethanol. Shell size measurements and eyespot identification, from microscopic inspection, were recorded for a subsample of specimens within 24 hours of preservation. Larval size was estimated by measuring width (perpendicular to shell hinge) and height (parallel to hinge) and treating larvae as ellipsoids, so that larval size is measured in surface area.

\subsubsection{Experimental setup}

All experimental observations were conducted in an environmental chamber at a constant temperature of $20^{\circ} \mathrm{C}$, in the dark. The observational tank was a $50 \mathrm{~mL}$ flat-sided plastic flask with an open top, filled with either 1$)$ ambient seawater $\left(20^{\circ} \mathrm{C}, 33 \mathrm{psu}\right)$ filtered to $1 \mu \mathrm{m}$, or 2$)$ oyster bathwater at identical temperature and salinity (see below for preparation method). Larvae swam into the flask via a gravity-assisted $1 \mathrm{~mL}$ pipette suspended above the flask, with the pipette tip just breaching the water surface; this method of introduction imposed no external downward momentum on larvae. Approximately 40-50 larvae were introduced to the tank for each observational period. The tank was illuminated from behind with a nearinfrared LED array light source (Olymstore, 12V, 2A, $850 \mathrm{~nm}$ ) and a monochrome camera (Hitachi KPF-120) recorded a $4 \times 5 \mathrm{~cm}$ 2-dimensional field of view, which encompassed a vertical cross-section of the entire flask volume. The bottom of the flask consisted of a smooth plastic surface with no biofilm, a poor settlement surface for oyster larvae [166]. The flask was also allowed to rest prior to the addition of larvae, and maintained at the same temperature as the environmental chamber, to minimize convective currents or other flow 
fields from forming.

For both experimental spawns, a subsample of larvae was exposed to the control filtered seawater (SW) and a subsample was exposed to the oyster bathwater (OB). Spawn 1 had larvae exposed at time $0,4,8$, and 12 hours post-competency, while Spawn 2 had larvae exposed at time $0,10,22,43$, and 64 hours post-competency. For both spawns, $t=0$ hours was defined to occur when $>50 \%$ of larvae displayed evidence of competency. At each time point, 5 replicate observations were taken for each of the SW and OB treatments, with each replicate entailing five minutes of video observation at 30 frames per second of larval motion in the field of view. Experimental images were saved as high resolution TIFF files $(1040 \times$ 1390 pixels) for subsequent larval tracking.

Oyster bathwater was prepared in a similar method to that described in Tamburri et al. [40]. Live, unwashed, adult C. virginica were purchased from a local vendor (The Clam Man, Falmouth, MA, USA) from a recent harvest of farmed oysters in Waquoit Bay (MA, USA). Individuals were measured to estimate shell surface area, and a set of oysters totaling $800 \mathrm{~cm}^{2}$ surface area were placed in a sterile plastic bucket with $4 \mathrm{~L}$ of aerated seawater filtered to $1 \mu \mathrm{m}$ (ambient $20^{\circ} \mathrm{C}, 33 \mathrm{psu}$ ). The bucket was covered and left undisturbed in the environmental chamber for 4 hours. Subsequently, the adults were removed and the bathwater filtered to $0.7 \mu \mathrm{m}$ through a glass microfiber filter. The bathwater was then divided into $50 \mathrm{~mL}$ aliquots and frozen at $-20^{\circ} \mathrm{C}$ until immediately prior to use.

\subsubsection{Larval tracking}

The methods for larval identification and tracking were adopted from Wheeler et al. [28, 29] and briefly summarized here. TIFF images were imported into LabVIEW 2013 (National Instruments) and average background intensity was subtracted. Using a fixed threshold particle size and intensity, larval centroid positions $(x, z)$ were recorded in the frame which they appeared. Centroid positional data were reconstructed into individual larval trajectories using a MATLAB script which tracked a larva from frame to frame according to a subsequent-frame tolerance distance radius set by the user (e.g. Figs. 6-1 a, c). Larval trajectories were truncated by five frames at the terminal points of the trajectories to avoid poor centroid estimates in cases where larvae passed laterally into and out of the focal plane. Positional probabilities were calculated by defining a grid in the $(x, z)$-space and determining the number of larvae appearing in each grid box over the total observational period, 
normalized by number of observations (e.g. Figs. 6-1 b, d). Instantaneous swimming velocities were computed using a central difference scheme of larval centroid positions in time, so that the velocity is defined centered in time between two adjacent frames. Vertical and horizontal distributions of position and velocity were constructed from mean positions and velocities of individual larval trajectories; these distributions were used for subsequent statistical analysis. Unlike the set-ups described in [28, 29], flow velocities in this small tank were minimal and therefore no effort was made to subtract local flow away from observed larval velocities.

\subsubsection{Statistical Analysis}

The average larval vertical position, calculated from larval tracking, in both the SW and OB treatments followed strongly non-normal distributions at all time periods, and as such we used a non-parametric Kruskal-Wallis test to assess the changes to the median vertical position in each seawater treatment over time. We assessed significant differences in vertical distributions between seawater treatments by pooling observations over all the time periods together and applying a Mann-Whitney test. We also used the same statistical tests to assess potential changes in horizontal position over time and between seawater treatments. If either the horizontal or vertical position was impacted by larval age or seawater treatment, we assessed the similarity or difference of the behaviors in the SW and OB treatments over time in that dimension. We used a Kruskal-Wallis test to diagnose the difference in position between the seawater treatments over the larval age.

In the event of a significant difference in larval position with seawater treatment and/or larval age, we investigated the effects of these variables on larval velocity. To do so, we repeated the same suite of statistical analyses: a Mann-Whitney test on velocity distributions for each seawater treatment, pooled across all larval ages, and a set of Kruskal-Wallis tests on individual seawater treatments over time, followed by a Kruskal-Wallis test on the difference in mean velocity between the seawater treatments over time.

Since larval size can impact swimming velocity in oyster larvae [93], we wanted to ensure that no size differences existed between the seawater treatments at any time. Larval surface area was recorded in a preserved sample of larvae at each time period in the experiment for both seawater treatments, and for each treatment, differences in larval size for larvae used in seawater treatments and over the experimental time scale were assessed using a 2-factor 
ANOVA. Because larvae were selected for each seawater treatment from a common pool immediately prior to use, we do not expect differences in size by treatment, though changes in size over the experimental time are possible. The proportion of competent larvae, denoted by the presence of an eyespot, was also estimated from the preserved sample of larvae. The change in proportion of competent larvae over time was assessed for each of the SW and OB treatments using a probit regression model. Like with size, we expect no difference in competency between the seawater treatments but increases in competency proportion over time is likely.

\subsection{Results}

\subsubsection{Larval position}

The presence of the chemical cue had a strong impact on larval vertical position in the water column in both Spawn $1(z=3.07$, rank sum $=524, p=0.002)$ and Spawn $2(z=4.88$, rank sum $=890, p<0.001)$ pooled across time post-competency. In Spawn 1, mean position in the SW treatment was $1.90 \pm 0.15 \mathrm{~cm}$ above the bottom, while in the OB treatment, mean position was $1.21 \pm 0.12 \mathrm{~cm}$ above the bottom. In Spawn 2, mean position in the SW treatment was $2.90 \pm 0.09 \mathrm{~cm}$ above the bottom, while in contrast, larvae in the OB treatment held a mean position $1.93 \pm 0.12 \mathrm{~cm}$ above the bottom. Seawater treatment had no impact on horizontal position for either Spawn $1(z=-1.74$, rank sum $=345, p=0.081)$ or Spawn $2(z=-0.42$, rank sum $=615, p=0.66)$. The behavior between seawater treatments differed qualitatively. In both treatments, larvae swam down out of the pipette tip towards the bottom, but in the SW treatment, larvae subsequently swam back upward in the flask. Some larvae swam helically upwards and occasionally sank downward before resuming swimming, and others encountered the surface and aggregated there (Fig. 6-1 a,b). Larvae explored a large proportion of the observed tank area during the observational period (Fig. 6-1 b). In the OB treatment, larvae swam downward in straight trajectories from the pipette (Fig. 6-1 c) and aggregated near the bottom, exhibiting some lateral motion near the bottom surface (Fig. 6-1 c,d).

Mean larval vertical position did not vary significantly with larval age in either seawater treatment in Spawn 1, but increased significantly at intermediate larval age in the Spawn $2 \mathrm{SW}$ treatment before decreasing toward the end of the experimental time. The Spawn 

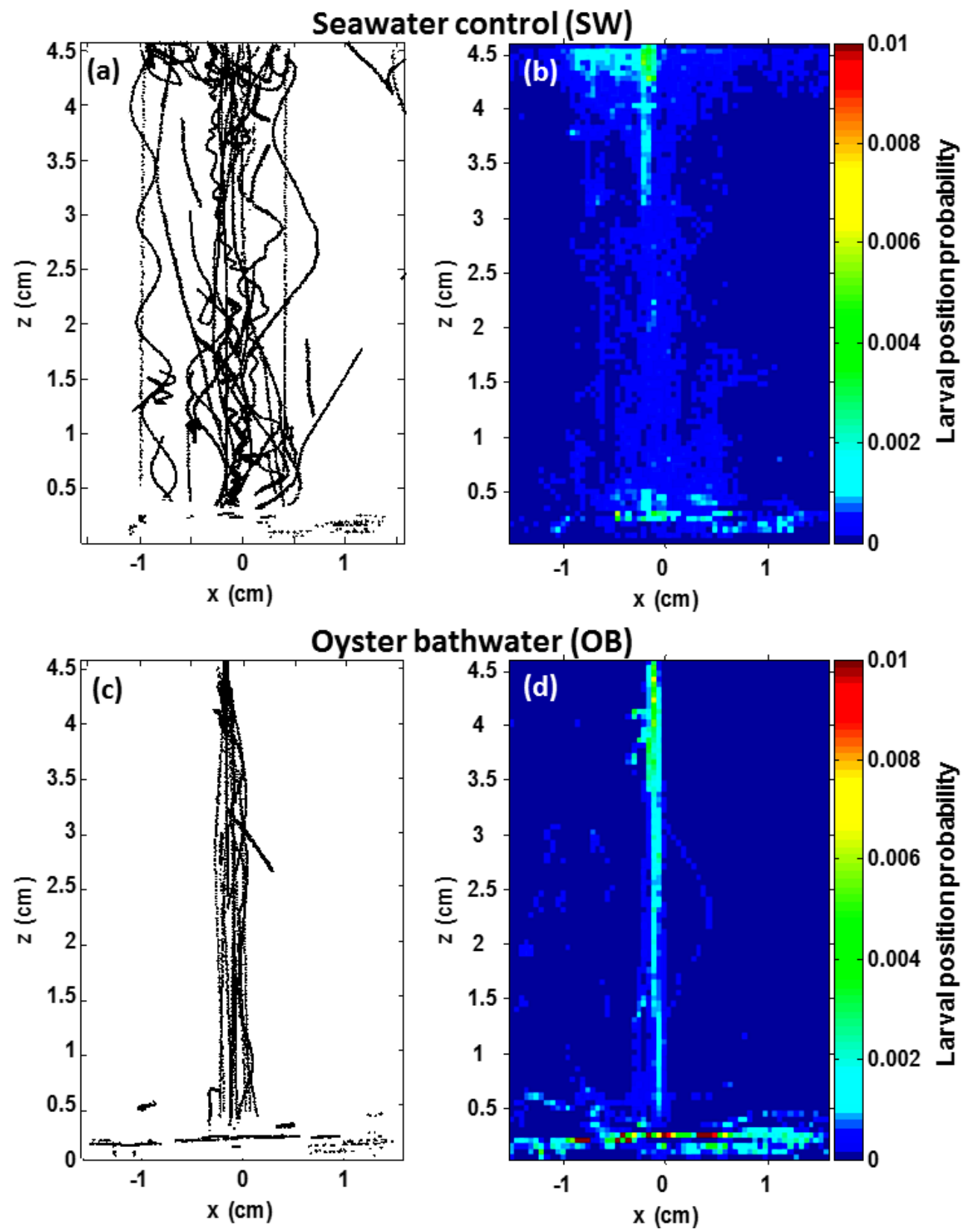

Figure 6-1: (Left) Sample representative trajectories from the SW treatment (a) and OB treatment (c). (Right) Color map of larval position probability for SW (b) and OB (d) treatments, pooling all five replicates from one observation period (specifically, Spawn 2 at $t=0$ hours). Note that the color bar is non-linear and equal for both seawater treatments. 
2 OB treatment showed no effect of larval age on vertical position (Fig. 6-2 a, Table 61). Larval horizontal position was unaffected by larval age in either seawater treatment (Table 6-2) and exhibited no coherent pattern with larval age. The difference in mean vertical position in the seawater treatments over larval age was non-significant in Spawn 1, but showed an increasing trend over the 12 hour window. In Spawn 2, the difference was likewise non-significant but yielded the same increase initially seen in Spawn 1, as well as a steady decrease over the remainder of the 64 hour experimental time window (Fig. 6-2 b, Table 6-3). Qualitatively, while larvae persisted in exploratory swimming behaviors in the SW treatment over time, their average proximity to the bottom increased, while the larvae in the $\mathrm{OB}$ treatment exhibited no positional change, suggesting that the behavior was becoming more similar between the two treatments. 

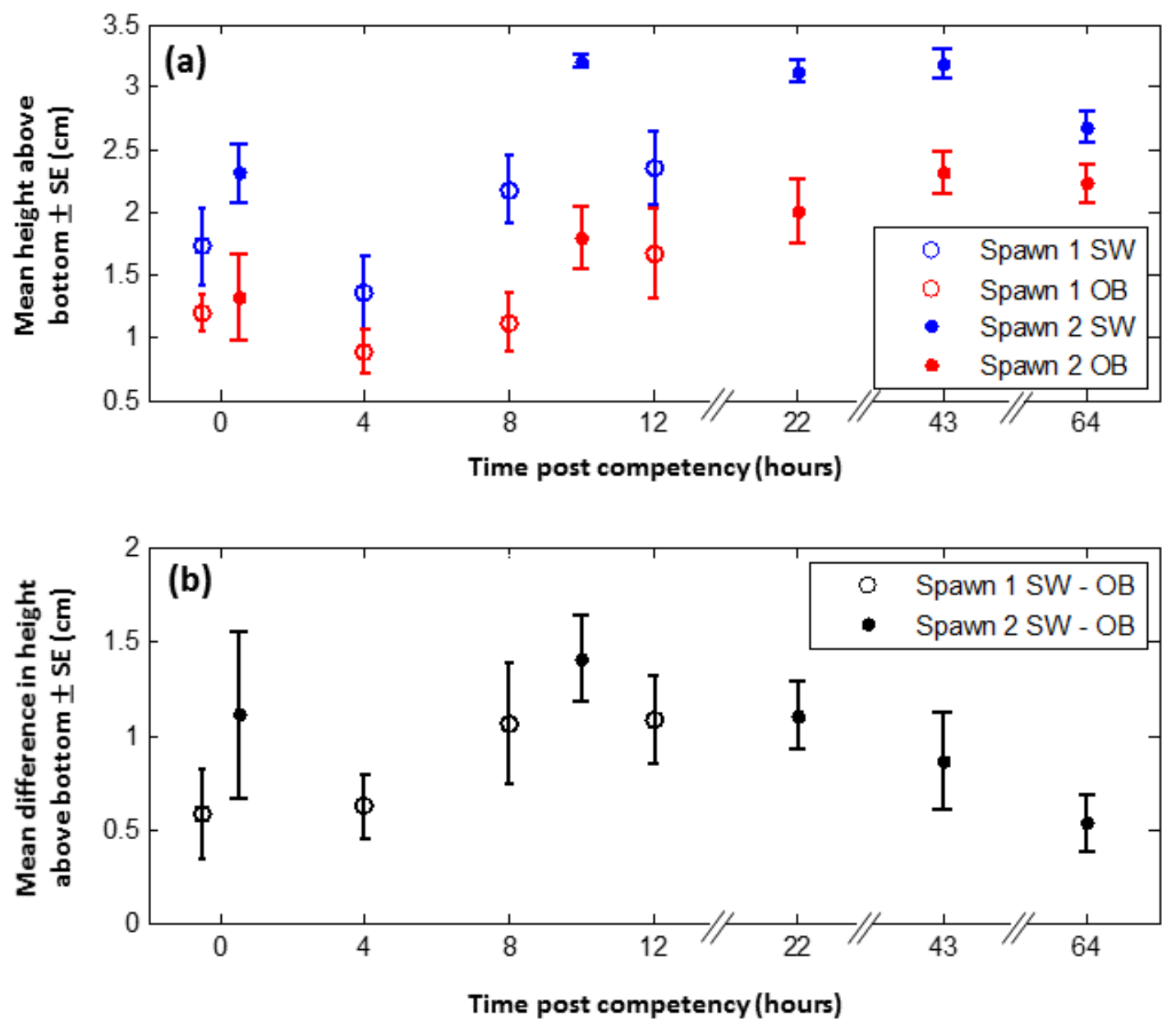

Figure 6-2: (a) Mean larval vertical position above the bottom with standard error bars with respect to time post competency, for Spawn 1 (open circles) and Spawn 2 (closed circles). Blue denotes SW treatments and red denotes OB treatments. (b) Difference in mean larval vertical position between SW and OB treatments over the competency period for Spawn 1 (open circles) and Spawn 2 (closed circles). Note that the time axis is non-linear for greater clarity.

\subsubsection{Larval vertical velocity}

The seawater treatment had a significant effect on vertical swimming velocity in both Spawn $1(z=4.42$, rank sum $=574, p<0.001)$ and Spawn $2(z=5.66$, rank sum $=930$, $p<0.001)$ pooled across time post competency. In Spawn 1, SW mean larval vertical swimming velocity was $-0.024 \pm 0.0016 \mathrm{~cm} / \mathrm{s}$, significantly greater than the OB swimming velocity of $-0.066 \pm 0.0075 \mathrm{~cm} / \mathrm{s}$. Likewise, in Spawn 2, SW mean larval vertical swimming 


\begin{tabular}{|l|l|l|l|l|l|}
\hline Source & SS & $d f$ & MS & $\chi^{2}$ & $P$ \\
\hline Spawn 1 SW & & & & & \\
Time post competency & 199.4 & 3 & 66.46 & 5.7 & 0.12 \\
Error & 465.6 & 16 & 29.1 & & \\
Total & 665 & 19 & & & \\
\hline Spawn 1 OB & & & & & \\
Time post competency & 145 & 3 & 48.33 & 4.14 & 0.24 \\
Error & 520 & 16 & 32.5 & & \\
Total & 665 & 19 & & & \\
\hline Spawn 2 SW & & & & & \\
Time post competency & 862.8 & 4 & 215.7 & 15.93 & $\mathbf{0 . 0 0 3}$ \\
Error & 437.2 & 20 & 21.86 & & \\
Total & 1300 & 24 & & & \\
\hline Spawn 2 OB & & & & & \\
Time post competency & 365.2 & 4 & 91.3 & 6.74 & 0.15 \\
Error & 934.3 & 20 & 46.71 & & \\
Total & 1299.5 & 24 & & & \\
\hline
\end{tabular}

Table 6-1: Kruskal-Wallis tests comparing the effects of time post competency on larval vertical position in both spawns and seawater treatments. Significance level is $\alpha=0.05$ and significant results are in bold.

\begin{tabular}{|l|l|l|l|l|l|}
\hline Source & SS & $d f$ & MS & $\chi^{2}$ & $P$ \\
\hline Spawn 1 SW & & & & & \\
Time post competency & 66.56 & 3 & 22.2 & 1.9 & 0.59 \\
Error & 598.4 & 16 & 37.4 & & \\
Total & 665 & 19 & & & \\
\hline Spawn 1 OB & & & & & \\
Time post competency & 123.4 & 3 & 41.13 & 3.53 & 0.31 \\
Error & 541.6 & 16 & 33.85 & & \\
Total & 665 & 19 & & & \\
\hline Spawn 2 SW & & & & & \\
Time post competency & 401.6 & 4 & 100.4 & 7.41 & 0.11 \\
Error & 898.4 & 20 & 44.92 & & \\
Total & 1300 & 24 & & & \\
\hline Spawn 2 OB & & & & & \\
Time post competency & 184.4 & 4 & 46.1 & 3.41 & 0.49 \\
Error & 1115.1 & 20 & 55.75 & & \\
Total & 1299.5 & 24 & & & \\
\hline
\end{tabular}

Table 6-2: Kruskal-Wallis tests comparing the effects of time post competency on larval horizontal position in both spawns and seawater treatments. Significance level is $\alpha=0.05$. 


\begin{tabular}{|l|l|l|l|l|l|}
\hline Source & SS & $d f$ & MS & $\chi^{2}$ & $P$ \\
\hline Spawn 1 & & & & & \\
Time post competency & 111 & 3 & 37 & 3.17 & 0.36 \\
Error & 554 & 16 & 34.62 & & \\
Total & 665 & 19 & & & \\
\hline Spawn 2 & & & & & \\
Time post competency & 378.8 & 4 & 94.7 & 6.99 & 0.13 \\
Error & 921.2 & 20 & 46.06 & & \\
Total & 1300 & 24 & & & \\
\hline
\end{tabular}

Table 6-3: Kruskal-Wallis tests comparing the effects of time post competency on the difference in larval vertical position between seawater treatments. Significance level is $\alpha=0.05$.

velocity was $-0.025 \pm 0.0022 \mathrm{~cm} / \mathrm{s}$, significantly greater than the $\mathrm{OB}$ swimming velocity of $-0.118 \pm 0.0139 \mathrm{~cm} / \mathrm{s}$. The exploratory nature of larval swimming in the $\mathrm{SW}$ treatment ensured that larvae swam both upwards and downwards, leading to an average velocity near 0 . In contrast, the larvae in the $\mathrm{OB}$ treatment swam almost exclusively downwards and hence maintained a strong negative mean velocity.

Mean vertical swimming velocity did not vary significantly with larval age in either seawater treatment for either Spawn 1 or Spawn 2 (Fig. 6-3 a, Table 6-4). Comparing Spawn 1 to Spawn 2, vertical velocities in the SW treatments were very similar, while velocities in the OB treatments exhibited more variability. The difference in vertical swimming velocities between seawater treatments, however, was significant in Spawn 2: the difference in velocity increased significantly to moderate larval age and then decreased towards the end of the experimental time scale (Fig. 6-3 b, Table 6-5). Spawn 1 exhibited no significant effect of larval age on the difference in swimming velocities (Table 6-5), but it did follow the same trend as Spawn 2, in increasing over short time scales (Fig. 6-3 b). 

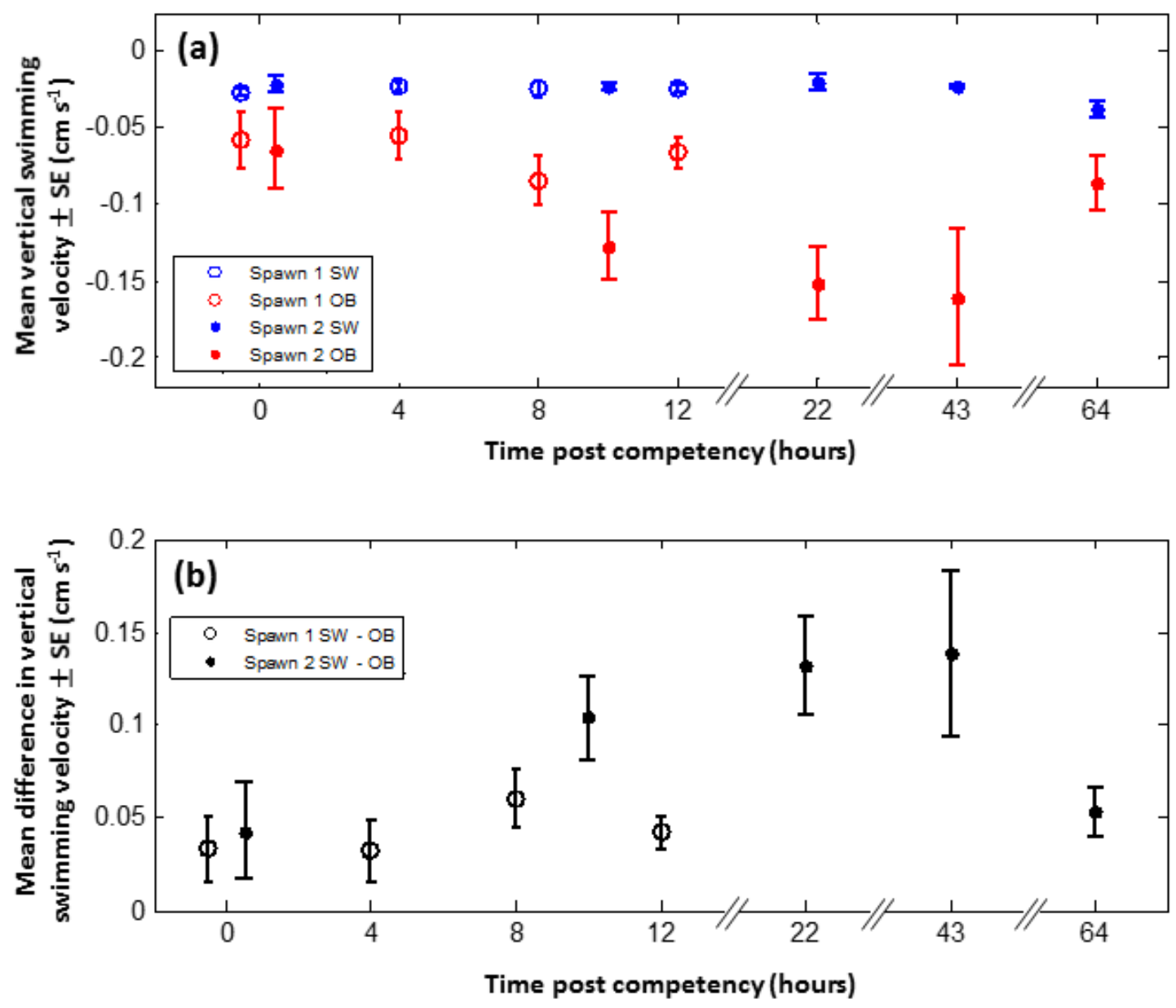

Figure 6-3: (a) Mean larval vertical velocity with standard error bars with respect to time post competency, for Spawn 1 (open circles) and Spawn 2 (closed circles). Blue denotes SW treatments and red denotes OB treatments. (b) Difference in mean larval vertical velocity between SW and OB treatments over the competency period for Spawn 1 (open circles) and Spawn 2 (closed circles). Note that the time axis is non-linear for greater clarity.

\subsubsection{Larval growth and competency}

Larval size did not differ between seawater treatments in either Spawn 1 or Spawn 2, but did increase significantly over the experimental time scale for both Spawn 1 (12 hours) and Spawn 2 (64 hours) (Fig. 6-4, Table 6-6). In Spawn 1, mean larval surface area increased from $2.63 \times 10^{5} \pm 8.50 \times 10^{3} \mu \mathrm{m}^{2}$ to $2.75 \times 10^{5} \pm 8.60 \times 10^{3} \mu \mathrm{m}^{2}$ over the course of the experiment, while in Spawn 2, surface area increased from $2.08 \times 10^{5} \pm 2.71 \times 10^{3} \mu \mathrm{m}^{2}$ 


\begin{tabular}{|l|l|l|l|l|l|}
\hline Source & SS & $d f$ & MS & $\chi^{2}$ & $P$ \\
\hline Spawn 1 SW & & & & & \\
Time post competency & 30.6 & 3 & 10.2 & 0.87 & 0.83 \\
Error & 634.4 & 16 & 39.65 & & \\
Total & 665 & 19 & & & \\
\hline Spawn 1 OB & & & & & \\
Time post competency & 109.4 & 3 & 36.46 & 3.13 & 0.37 \\
Error & 555.6 & 16 & 34.72 & & \\
Total & 665 & 19 & & & \\
\hline Spawn 2 SW & & & & & \\
Time post competency & 362.8 & 4 & 90.7 & 6.7 & 0.15 \\
Error & 937.2 & 20 & 46.86 & & \\
Total & 1300 & 24 & & & \\
\hline Spawn 2 OB & & & & & \\
Time post competency & 448 & 4 & 112 & 8.27 & 0.08 \\
Error & 934.3 & 20 & 42.57 & & \\
Total & 1299.5 & 24 & & & \\
\hline
\end{tabular}

Table 6-4: Kruskal-Wallis tests comparing the effects of time post competency on larval vertical velocity in both spawns and seawater treatments. Significance level is $\alpha=0.05$.

\begin{tabular}{|l|l|l|l|l|l|}
\hline Source & SS & $d f$ & MS & $\chi^{2}$ & $P$ \\
\hline Spawn 1 & & & & & \\
Time post competency & 93.4 & 3 & 31.13 & 2.67 & 0.44 \\
Error & 571.6 & 16 & 35.72 & & \\
Total & 665 & 19 & & & \\
\hline Spawn 2 & & & & & \\
Time post competency & 549.2 & 4 & 137.3 & 10.14 & $\mathbf{0 . 0 3}$ \\
Error & 750.8 & 20 & 37.54 & & \\
Total & 1300 & 24 & & & \\
\hline
\end{tabular}

Table 6-5: Kruskal-Wallis tests comparing the effects of time post competency on the difference in larval vertical velocity between seawater treatments. Significance level is $\alpha=0.05$ and significant results are in bold. 
to $2.63 \times 10^{5} \pm 4.56 \times 10^{3} \mu \mathrm{m}^{2}$. In contrast to surface area, Spawn 1 showed no significant increase in competency over time for the SW $(t=0.49, p=0.62, d f=2)$ nor OB treatments $(t=1.04, p=0.29, d f=2)$ (Fig. 6-5 a). The competency proportion increased from 0.571 to 0.733 and 0.533 to 0.666 in the SW and OB treatments, respectively. Over the longer timescale to which Spawn 2 was subjected, both the SW and OB treatments showed significant increases over the full competency period $(t=2.78, p=0.005, d f=3)$ and $(t=2.65, p=0.007, d f=3)$ (Fig. 6-5 b), increasing from 0.615 to 1 in the SW treatment and from 0.666 to 1 in the OB treatment. Because neither larval size nor competency proportion differed significantly between the seawater treatments over time, we conclude that larval size (with associated changes to swimming capabilities) did not cause the differences in behavior observed between the seawater treatments.
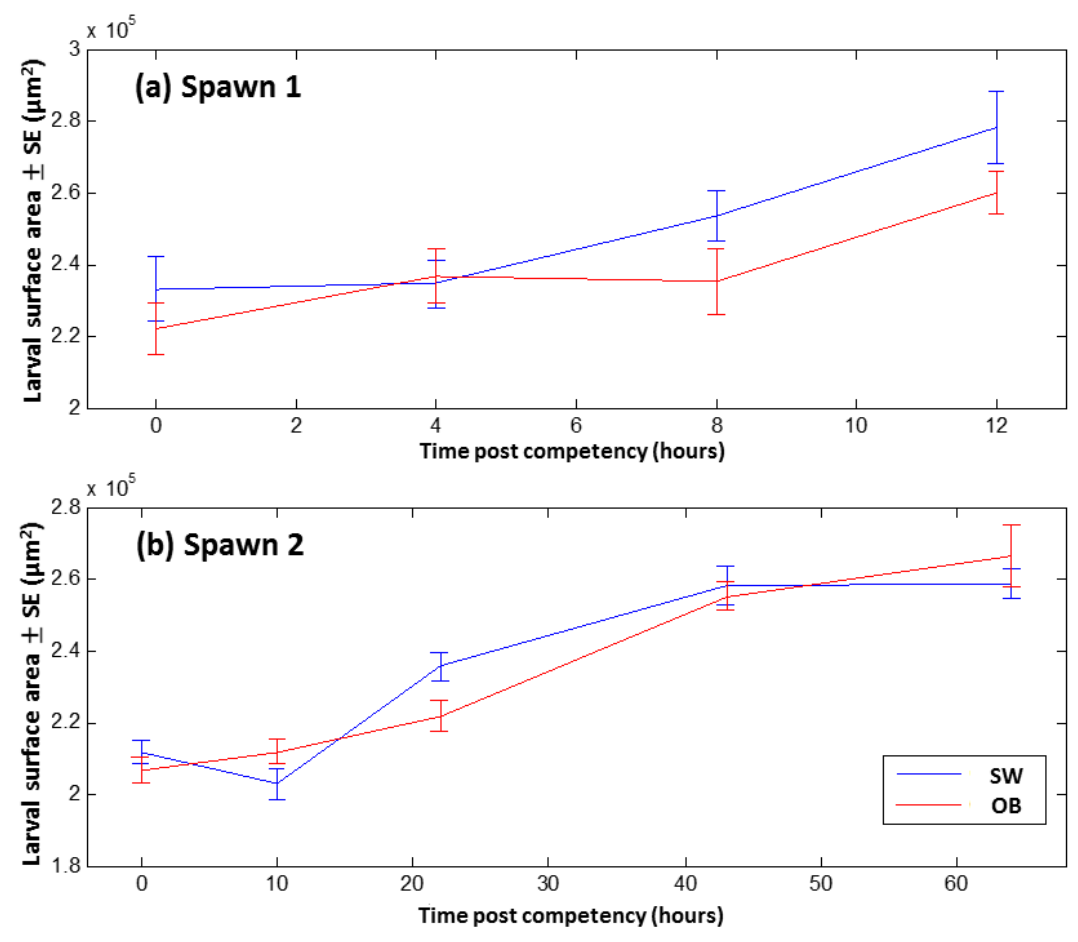

Figure 6-4: Mean larval surface area with standard error bars over the experimental time scale for Spawn 1 (a) and Spawn 2 (b). Blue lines and red lines denote the SW and OB treatments, respectively. 


\begin{tabular}{|l|l|l|l|l|l|}
\hline Source & SS & $d f$ & MS & $F$ & $P$ \\
\hline Spawn 1 & & & & & \\
Cue presence & $2.60 \times 10^{9}$ & 1 & $2.60 \times 10^{9}$ & 3.13 & 0.084 \\
Time post competency & $1.97 \times 10^{10}$ & 3 & $6.58 \times 10^{9}$ & 7.9 & $\mathbf{0 . 0 0 3}$ \\
Interaction & $4.32 \times 10^{9}$ & 3 & $1.44 \times 10^{9}$ & 1.73 & 0.1764 \\
Error & $3.33 \times 10^{10}$ & 40 & $8.33 \times 10^{9}$ & & \\
Total & $6.00 \times 10^{10}$ & 47 & & & \\
\hline Spawn 2 & & & & & \\
Cue presence & $3.10 \times 10^{6}$ & 1 & $3.10 \times 10^{6}$ & 0.01 & 0.9182 \\
Time post competency & $7.32 \times 10^{10}$ & 4 & $1.82 \times 10^{10}$ & 62.47 & $\mathbf{0 . 0 0}$ \\
Interaction & $4.29 \times 10^{9}$ & 4 & $1.07 \times 10^{9}$ & 3.66 & $\mathbf{0 . 0 0 7}$ \\
Error & $3.51 \times 10^{10}$ & 120 & $2.93 \times 10^{8}$ & & \\
Total & $1.12 \times 10^{11}$ & 129 & & & \\
\hline
\end{tabular}

Table 6-6: Two-factor ANOVA comparing the effects of the cue presence (SW versus OB) and time post competency on larval surface area. Significance level is $\alpha=0.05$ and significant results are in bold.
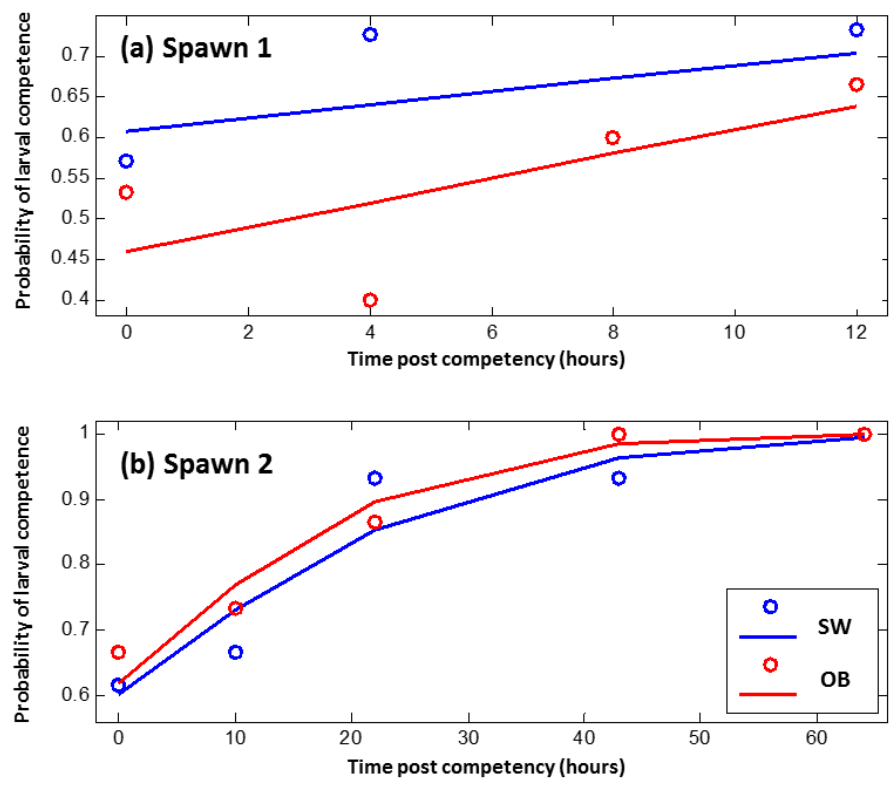

Figure 6-5: Probability of larval competence over experimental time scale for Spawn 1 (a) and Spawn 2 (b). Blue and red circles represent observed competence proportion in sampled preserved larvae in the SW and $\mathrm{OB}$ treatments, respectively, and the blue and red curves are probit regression models fit to the data. 


\subsection{Discussion}

The desperate larva hypothesis would suggest that young larvae might initially ignore a chemical inducer before accepting it as a settlement cue as they aged; in this case, larval behavior between cue and cue-less larvae would be similar initially and diverge with time. Alternatively, larvae might accept a chemical inducer as a settlement cue regardless of age, and larval desperation would manifest as cue-less larvae eventually settling even in the absence of a cue. In this case, larval behavior between cue and cue-less larvae would initially appear different and converge with time. In this study, larval oysters demonstrated a distinct behavior when exposed to oyster bathwater over the entire time range of larval competency, swimming straight downward in the flask and maintaining a low average vertical position near the bottom. In contrast, larvae provided with no settlement cue exhibited exploratory swimming behavior: swimming upward in helices, diving, and maintaining an average position much higher in the flask during early stages of competency. At longer time scales, older larvae without a settlement cue began to behave more similarly to the larvae exposed to bathwater, as their average vertical positions and vertical swimming velocities began to converge. (In a similar qualitative observation, larvae maintained in culture buckets for the full duration of the experiment likewise began to aggregate near the bottom over time). There was no difference in body size or competence to account for differences or lack thereof in larval behavior in the two seawater treatments, leading us to conclude that larvae were actively modifying their swimming behavior with age when exposed to no settlement cue. This decreased selectivity for settlement habitat follows our second interpretation of the desperate larva hypothesis.

Mean larval vertical position decreased over long time scales in the SW treatment after an initial significant increase over short time scales in Spawn 2, with the short time scale increase reflected in Spawn 1. The dome-shaped response in vertical position with time is similar to pre-settlement swimming patterns documented in other larval invertebrates: the bryozoan Bugula neritina spends more time exploring near the bottom at early and late stages of competency, and during intermediate stages, spends more time swimming [167]. In the $\mathrm{OB}$ treatment, in contrast, there was no change in height over time in either spawn. This led to the (albeit non-significant) decrease in the difference between mean position in the SW and OB treatments over time, highlighting that the behavior of the larvae in the 
two treatments became more similar.

The larvae increased significantly in size as they aged, but we do not believe that an increase in negative buoyancy alone explains the decrease in mean vertical position, as there is no corresponding decrease in vertical position observed over time in the $\mathrm{OB}$ treatment (which contained larvae of comparable size). Further, average vertical swimming velocity at late stages was well within the oyster larval capabilities [93, 28], suggesting that larvae could actively maintain the higher position in the water column, and were not constrained by their own swimming limitations.

There is considerable difference in body size between Spawn 1 and Spawn 2, which clearly plays a role in determining the mean height within the spawn, as can be seen in the interspawn height difference at the early time scales. However, we observe that this impacts both SW and OB treatments, so again, a change over time in one treatment but not the other is likely not due only to changes in body size. Further, it is interesting to note that while the vertical positions at early time depend strongly on spawn, the differences between the positions in the two seawater treatments are very similar in both spawns, suggesting that height discrepancy between larvae exposed to the different treatments is invariant to body size. If so, this would suggest that response to no cue versus cue holds for larvae over a range of sizes, relevant for a species where body size is strongly dependent on temperature, salinity, and food availability [6].

Larval position in the water column can also be influenced by flow [25, 26, 27, 28, 29] and sound [30] for oyster larvae. For other larval invertebrates, the presence of predators $[36,37]$ and light $[31,32,33,34,35]$ can also impact larval vertical distributions. None of these factors were present in our experiment, though it would be interesting and potentially ecologically relevant to observe how our results would change with the addition of another factor. For instance, many invertebrate larvae display ontogenetic shifts in phototaxis, becoming negatively phototactic with age $[138,35]$. The addition of an overhead light might induce more aggregation of larvae near the bottom as they age, which would render the difference between the seawater treatments more pronounced. However, an overhead light producing an illumination gradient could certainly be interpreted by larvae as a settlement cue, and in the present experiment, we endeavored to give the control larvae an environment devoid of any cues for settlement.

We observed no difference in the mean horizontal position between the seawater treat- 
ments, nor with larval age. This result reflects the left-right symmetry of the larvae for directional swimming, which is not the case in the vertical direction due to the negative buoyancy of the larvae. The lack of horizontal directional bias also indicates there were no transient horizontal flows in the tank to advect the larvae. However, it is worthwhile to note that the variance of the horizontal position distributions were in general larger in the SW than $\mathrm{OB}$ treatments (frequently $>2$ times larger), reflecting the greater range of the larvae swimming behavior in the absence of a settlement cue.

In both spawns, larvae in the SW treatments maintained a vertical swimming velocity just below zero, while in the $\mathrm{OB}$ treatments the swimming velocities were significantly lower. Recalling that larvae are negatively buoyant, larvae with slightly negative swimming velocities (such as in the SW treatment) were actually actively swimming upward, which was countered by their downward passive sinking velocity. It is not inconsistent to observe different mean heights in the water column while maintaining similar swimming velocities, as observed for the SW treatments in Spawns 1 and 2, since larvae could maintain a vertical position (i.e., zero vertical velocity) at different vertical positions. Vertical velocity did not appear to be affected strongly by body size, as indicated by very similar velocities in the SW treatments for Spawn 1 and 2.

Larval swimming velocities also became more similar between the seawater treatments as larvae aged. Larvae maintained negative downward velocities over time, and this, coupled with an overall decrease in mean vertical position, suggests that larvae engaged less in exploratory swimming behavior in the absence of a settlement cue, and began to behave more like larvae exposed to a strong settlement cue. This is evidence that oyster larvae behave as predicted by the desperate larva hypothesis. The question of why larvae become 'desperate' is interesting: the older oyster larvae which we observed were in good condition and well fed, so what triggers their desperation to settle? If larvae choose to remain in the water column, they are exposed to its dangers: larvae in late stages remain vulnerable to predators [168, 169] and infection [170], as well as transport away from coastal settlement sites [171]. Further, patchiness of nutrients $[172,173]$ makes a continued food supply uncertain. Nutrient limitation has been investigated as a possible trigger for larval desperation with inconclusive results. Toonen and Pawlik [174] found no decrease in habitat selectivity in the gregarious larvae of the serpulid polychaete Hydroides dianthus with age and feeding regime, but Botello and Krug [39] reported that a starvation regime made the facultative 
planktotroph opisthobranch Alderia more sensitive to chemical settlement cues. In our experiment, larvae were protected from the detrimental effects of starvation, predation, and infection, and as such we minimized the potential negative effects of these on larval behavior. If larval desperation is not induced by these factors, it might have been induced by the perpetual lack of a settlement cue throughout the competency period. Larval oysters are gregarious settlers, and preferentially settle on adult oyster reefs, which have historically spanned hundreds of meters to kilometers squared at densities of $>150$ adults $\mathrm{m}^{-2}$ [175]. Chemical cues released by the adults (analogous to our oyster bathwater) can be advected and turbulently mixed off the rough bottom topography (e.g., [118]) and are detectable by larvae at extremely low concentrations (down to $10^{-10} \mathrm{M},[40]$ ). In our experiment, old larvae had detected no cues for in excess of 60 hours: such a long search period with no settlement signal might indicate to larvae that there was no suitable settlement habitat even remotely proximate. The consistent absence of the settlement cue over a long time may have induced the larval desperation to settle in sub-standard conditions.

The larval oyster response to exudates from adult oysters is immediate downward swimming to the bottom, and this behavior persists over the entirety of the competency period. The contrast between this behavioral mode and the normal exploratory swimming indicates that the presence of adult oysters supersedes any further exploration of the water column by larvae. The slow convergence of the cue-less larvae from exploratory to settlement behavior indicates that larvae will persist in searching for a better habitat for multiple days, but will ultimately opt for the uncertain future of settlement and recruitment in a poor benthic site.

\subsection{Acknowledgements}

We thank Susan Mills for experimental assistance, Dave Kulis for oyster bathwater production, and gratefully acknowledge the Aquaculture Research Corporation (Dennis, MA, USA) and the Martha's Vineyard Shellfish Group (Martha's Vineyard, MA, USA) who provided the larvae used in this study. Thanks especially to Karen Chan and Joseph Fitzgerald for helpful discussions and manuscript comments. Funding was provided by NSF grant OCE0850419, NOAA Sea Grant NA14OAR4170074, grants from WHOI Coastal Ocean Institute, discretionary WHOI funds, a WHOI Ocean Life Fellowship to LM, and a Grove City College Swezey Fund Grant to EA. 


\section{Chapter 7}

\section{Discussion}

\subsection{Summary}

The primary aim of this thesis was to understand how larval invertebrates respond to environmental cues during their pelagic life stages, and how these responses may change with age. Larval behavioral responses to water column cues can affect their vertical position in the water column during pre-settlement stages, impacting dispersal and transport. During competency, behavioral responses may allow larvae to identify and investigate suitable settlement habitats, or avoid poor settlement habitats. Behavior, as mediated by age and environmental cues, can thus directly impact larval survival and success. A mechanistic understanding of the links between physical and chemical cues and larval behavior is also highly relevant for modelling and predictive studies; fluid flow, for instance, can be understood theoretically by a governing set of equations of motion, while larval behavior can only be observed experimentally. However, if a relationship between turbulence and larval behavior can be elucidated, then a model of larval behavior over a broad range of realistic environmental conditions becomes much more tractable.

The goal of this thesis was to directly experimentally address the following set of questions:

- Chapter 2: How do age and morphology impact swimming behavior in pre-competent sea urchin larvae in turbulence?

- Chapter 3: How do competent oyster larvae respond to turbulence, and what does this response imply about turbulence as a larval settlement cue? 
- Chapter 4: What particular hydrodynamic conditions trigger diving in competent oyster larvae?

- Chapter 5: How do interacting physical cues (light and turbulence) impact swimming behavior in competent oyster larvae?

- Chapter 6: How do conspecific chemical settlement cues influence swimming behavior in oyster larvae over the full competency period?

In Chapter 2, I investigated swimming behavior in two pre-settlement life stages of the purple urchin under a range of turbulence conditions in a grid-stirred tank. Younger, fourarmed pluteus larvae swam more slowly but were more stable in their preferred vertical orientation than older, six-armed larvae, and this effect was enhanced by increased turbulence intensity. This ontological change in stability suggests that larvae in different life stages could be hydrodynamically separated in turbulence and thus undergo stage-specific transport.

In Chapter 3, I examined how the swimming behavior of competent larval oysters changed under a broad range of turbulence conditions in a grid-stirred tank. While turbulence had previously been observed to induce sinking and potentially settlement in other invertebrate larvae, I found that oyster larvae persisted in upward swimming under a wide range of turbulence intensities. Further, the larval dive response, relatively common in low turbulence conditions, disappeared at high turbulence. I concluded that turbulence alone did not induce a settlement response in oyster larvae.

In Chapter 4, I linked the previously observed dive response in competent larval oysters to local hydrodynamic conditions. By comparing diving versus non-diving larvae and their respective hydrodynamic histories, I determined that diving larvae experienced anomalously high fluid acceleration over short time scales prior to the dive. From this analysis, I produced a behavioral parameterization which explicitly predicts the larval dive probability as a function of fluid acceleration, a useful framework for further modelling study. This study gave the first evidence for an active, direct larval response (diving) to a local hydrodynamic cue (fluid acceleration).

In Chapter 5, I investigated the potential for interactive effects of light and turbulence on competent larval oyster behavior. I found that light did not impact larval vertical swimming over a range of turbulence intensities in a grid-stirred tank. However, exploratory and 
predator avoidance behaviors (helical swimming and diving) increased in light in certain turbulence regimes. Thus, while light and turbulence together did not induce settlement responses in larvae, they did encourage swimming behaviors that would likely increase larval survivorship while larvae remained in the plankton.

In Chapter 6, I examined the role of larval age in mediating responses to a strong settlement cue (adult oyster bathwater) and to no settlement cue in still water. The desperate larva hypothesis suggests that as larval energy reserves are depleted, larvae will display decreased settlement habitat selectivity, that is, they will accept a substandard settlement cue or even no settlement cue. I found that oyster larval behavior became more similar between the two cue conditions as the competency period progressed, suggesting that oyster larvae may indeed demonstrate desperation with age.

\subsection{Synthesis}

This thesis presents a view of marine larval ecology in which larval invertebrate behavior is conceptualized as a probabilistic response to two distinct sets of primary drivers: external environmental cues and larval aging (Figure 1). External environmental cues explored in this thesis are turbulence (and its components), light, and adult oyster exudates. Larval aging is considered on two time scales with two corresponding facets of aging. During the planktonic larval stages, aging is addressed through changes to larval morphology, and during competency, it is addressed through changes in larval selectivity for settlement habitat. External cues and aging collectively drive larval behavioral responses, which can then be interpreted from an ecological viewpoint as 1) exploratory or 2) settlement behaviors with associated impacts on dispersal and successful recruitment, respectively. To demonstrate the conceptual framework, I will first discuss work that primarily falls along the left hand path of the figure (external environment $\rightarrow$ larval behavior), and then work that primarily falls along the right hand path of the figure (larval aging $\rightarrow$ larval behavior). Then, I will discuss how both paths can be considered simultaneously, to identify simultaneous (and interactive) effects of both external cues and aging on behavior. 


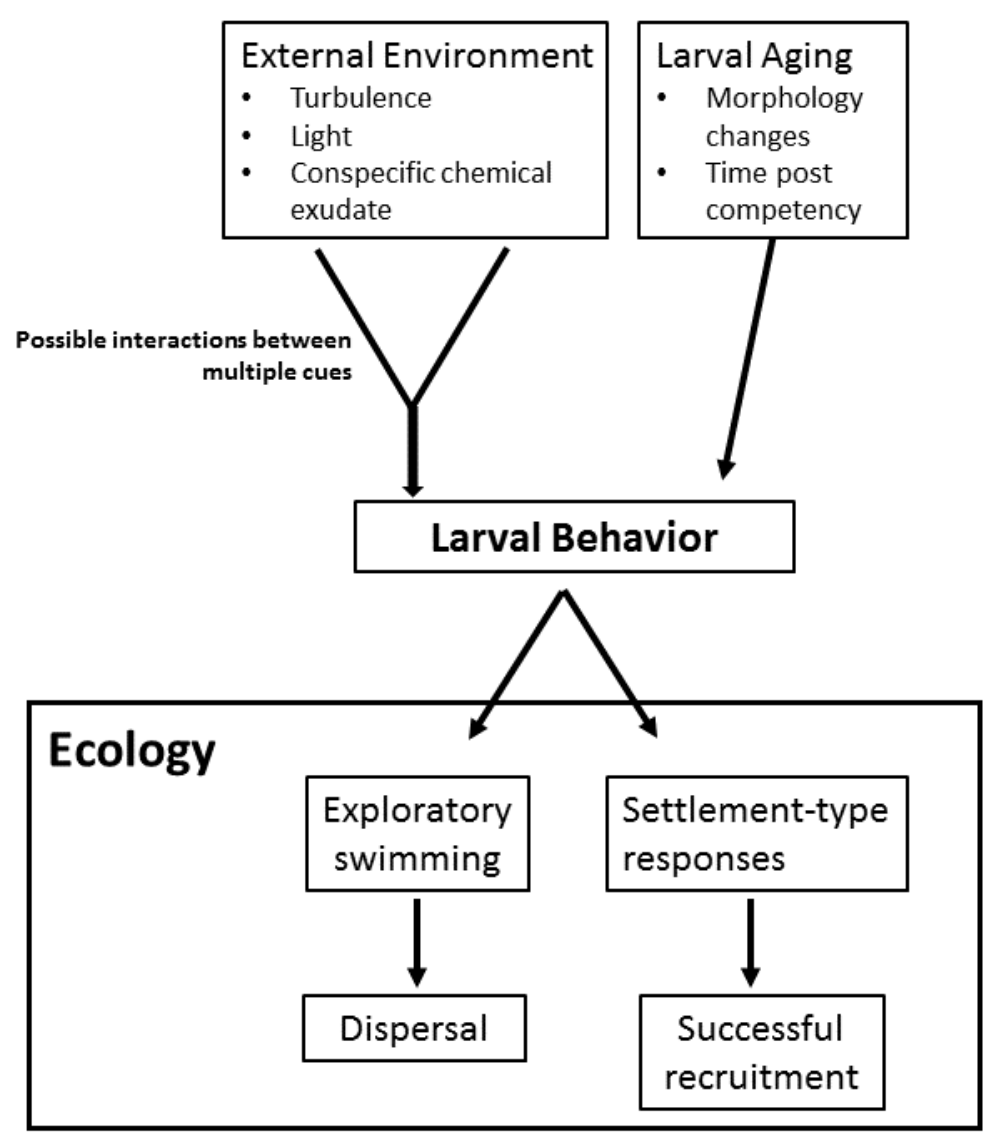

Figure 7-1: A schematic representation of our proposed framework of larval behavior, driven simultaneously by external cues and larval aging. Behavioral responses can be interpreted through their impact on larval ecology through changes to dispersal and recruitment.

\subsubsection{External environmental cues affect larval behavior}

Following the left hand path (Fig. 7-1), mapping external environment to larval response, consider the acceleration trigger to the dive response (Chapter 4) as an example. This study represents a case where the conceptual model for larval behavior was initially hypothesized as

$$
\text { behavior }=f(\text { turbulence }) \text {. }
$$

Through subsequent analysis of experimental data, the general behavior term was expressed in binary terms as a dive or non-dive, to distinguish the diving larvae for further investiga- 
tion. The conceptual model was refined into a probabilistic equation

$$
\operatorname{Pr}(\text { dive occurs })=f(\text { turbulence })
$$

and further investigation of components of turbulence detectable by larvae revealed that larvae were more likely to dive after experiencing an anomalously high fluid acceleration event so that

$$
\operatorname{Pr}(\text { dive occurs })=f(\text { acceleration }) \text {. }
$$

Because I could draw from my experimental data the average accelerations experienced by larvae pre-dive, I could determine the conditional probability of observing a given average acceleration event $|\mathbf{a}|$,

$$
\operatorname{Pr}(|\mathbf{a}| \mid \text { dive occurs })=F \text {, }
$$

and thus infer a function $G$ such that

$$
\operatorname{Pr}(\text { dive occurs }|| \mathbf{a} \mid)=G \text {. }
$$

The function $G$ represents a direct probabilistic mapping between the external environment and the larval response. My subsequent ecological interpretation was that transient high acceleration events (such as those occurring in an oscillating flow field over a rough substrate, like an oyster bed; [124] would signal to larvae in the water column to engage rapid downward motion (i.e., diving) to investigate the substrate for settlement suitability. Alternately, the fluid acceleration anomaly could be interpreted by larvae as the suction feeding current of a predator $[112,113,114]$ and the subsequent dive could be an escape response.

The dive-response study (Chapter 4) represents a quantitative mapping of a single environmental cue to a larval response, but in the field, it is likely that larvae experience and react to multiple cues simultaneously. To address the possible interactions between two cues I examined the changes to larval swimming when exposed to a range of turbulence intensities in dark versus light conditions (Chapter 5). I chose light as a complementary external cue to turbulence because I thought they might induce conflicting directional swimming responses: I previously observed competent oyster larvae to swim upwards in turbulence (Chapter 3), and light induces a downward swimming response (negative phototaxis) in other competent 
larvae, specifically ascidian and polychaete larvae $[138,35]$. However, the oyster larvae displayed no strong phototactic directional swimming response (either positive or negative), and hence there was no strong interactive effect of the two cues on directional swimming. It would be worthwhile and interesting, however, to observe a strongly phototactic larvae responding to these two cues simultaneously. Oyster larvae did however exhibit behavioral changes in light that were not directly related to directional swimming, and I will return to that point later in the discussion.

\subsubsection{Larval aging affects larval behavior}

Following the right hand path (Fig. 7-1), consider for example the competent oyster larvae exposed to no settlement cue in still water (Chapter 6). In that experiment, larvae were exposed to no external cues: they swam under their own power into a still water tank of filtered seawater of fixed temperature and salinity, with a bottom composed of smooth plastic. Over 64 hours of competency, larval behavior changed significantly. Newly competent larvae spent more time low in the water column, while larvae of intermediate competency engaged in exploratory swimming higher in the water column, and shifted back towards the bottom as they aged. Because the experiment controlled for all other effects, larval aging likely determined these behavioral changes.

\subsubsection{Interactive effects of age and external cues on larval behavior}

In the previous two sections, I focused on work that highlighted one driver of larval behavior over the other, but in reality, both pathways of influence are likely to be active simultaneously. The simultaneous consideration of both external cues and larval age can in fact give great insight into why larvae behave the way they do, from an ecological perspective. In the last example, for instance, I described the behavioral changes occurring in competent oyster larvae driven solely by larval aging. It is clear that behavioral changes occur even during this brief competency time window, but the ecological interpretation of these changes is aided by a point of comparison. Using larvae exposed to a chemical settlement cue (Chapter 6), I established a baseline set of distributions for position and swimming velocity in settling larvae. In comparing distributions for cue and cue-less larvae, I determined that aging and chemical cues have a similar impact on larval behavior, lending support to the desperate larva hypothesis. This ecological interpretation was made possible by an understanding of 
both how age and an external cue can impact behavior.

The interactive effect of age and external cues on larval behavior is also not restricted to the competency period, where sensitive behavioral changes occur over a short competency time window as larvae become desperate. There are ecological ramifications to such interactions during the pre-settlement stages, when aging manifests as changing morphology during successive larval stages. This is immediately obvious from my observations of larval urchins in turbulence (Chapter 2). Younger, four-armed pluteus larvae and older six-armed larvae displayed distinct swimming characteristics: older larvae swam faster but were more likely to be reoriented in flow, and this effect was exacerbated by increasing turbulence intensity. Larval swimming was thereby modulated simultaneously by both age and turbulence, leading me to speculate that the ontological shift in morphology-flow interactions could drive age and stage specific transport during the planktonic period.

It is also of ecological interest to consider the circumstances under which one of the two drivers dominates the larval response. For instance, when examining the swimming responses of larval oysters in light and turbulence (Chapter 5), larval age played a strong role in directional swimming. Larvae in early stages of competency were likely to swim upwards, becoming less likely to do so as time progressed. Age had a more dominant effect on directional swimming than did turbulence, and light had no effect at all. This led us to conclude that competent oyster larvae were not phototactic, and also that age drove downward swimming responses (as in Chapter 6). However, there were other behaviors apart from directional swimming which changed in the presence of light. Helical swimming and diving were both slightly more common in light than darkness, and these behaviors did not appear to change with age. External cues may have a strong effect on behavior regardless of age when the behavior in question has a strong impact on continued survivorship. Oyster larvae have a photosensitive eyespot [103] and visual predators [140], and both helical swimming and diving are speculated to be anti-predatory behaviors (Chapter 4, and [139]). Larvae engaging predator avoidance strategies in light would be beneficial in all life stages, and this may account for why the response does not change with age.

\subsubsection{Extensions}

The interactive effects of external environmental cues and age on larval behavior offer a diverse set of future research directions, but I highlight two specific extensions here relating 
to larval urchin reorientation dynamics and larval oyster sensitivity to chemical cues.

\section{Urchin reorientation dynamics}

Larval sea urchins swim using long, stiff ciliated arms, and they develop more pairs of arms as they mature. They swim in the direction that their arms are oriented, and swim preferentially in an upward, vertical orientation. I previously observed (Chapter 2) that larvae are reoriented away from vertical in turbulence, and this destabilization in flow is more pronounced in the older, six-armed larvae. However, in addition to swimming and orientation data for larvae in flow, I also have local flow data around individual larvae, meaning that I can attempt to link larval reorientation directly to local vorticity, and parameterize a model for larval gyrotaxis. One challenge in parameterizing such a model is the noise introduced to the parameterization by the unsteady forcing of the turbulent flow. To address

this, I conducted experiments with urchin larvae in a microscale "flip" chamber, where the experimental chamber is inverted and larvae permitted to reorient. Observations of larval orientation angles were taken once transient fluid rotations ceased, to avoid any effect of forcing by the flow. These experiments were conducted with two species of larvae across four, six, and eight armed stages. Ultimately, the resultant mechanistic model will directly link fluid vorticity to larval reorientation, and help to predict at what turbulence intensities larvae can no longer maintain preferred swimming strategies and will have no active control over dispersal during the planktonic period. This type of mechanistic understanding can help us link small-scale fluid-morphology interactions to broad scale stage-specific transport processes, which ultimately influence where larvae settle.

\section{Oyster sensitivity to chemical cues}

One interpretation of the desperate larva hypothesis is that larvae will ultimately settle even in the absence of a settlement cue, or in the presence of a very poor cue. This is supported by my observations of oyster swimming behavior over the full competency period (Chapter 6). An alternate, equally valid interpretation of the hypothesis is that larvae become more sensitive to settlement cues with age, so that older larvae detect lower cue concentrations than younger larvae $[155,161,165]$. One may consider the desperate larva hypothesis in the following framework: two larval behavioral regimes, classified as "non-settlement" and "settlement", are mediated by larval age and the concentration of the chemical cue (Fig. 
7-2). Prior to competency, the concentration of the cue necessary to induce settlement is functionally infinite (green shading), while during competency, if the concentration is sufficiently high, larvae settle (blue shading). In the absence of a sufficiently high cue, larvae do not settle and continue to swim (red shading). The cue concentration necessary to induce settlement (thick black curve) decreases as larvae age through competency (as they decrease cue selectivity). In my work, I determined the age at which settlement-like behavior developed in the absence of cue, denoted in the schematic as the $x$-intercept of the thick black curve. A further verification of the desperate larva hypothesis would be to experimentally determine further points on the curve. To do this, I would repeat my previous experiments with serial dilutions of oyster bathwater, in order to expose larvae to a range of cue concentrations. If oyster larvae responded to lower concentrations of cue over the competency window, this would offer further evidence for the desperate larva hypothesis.

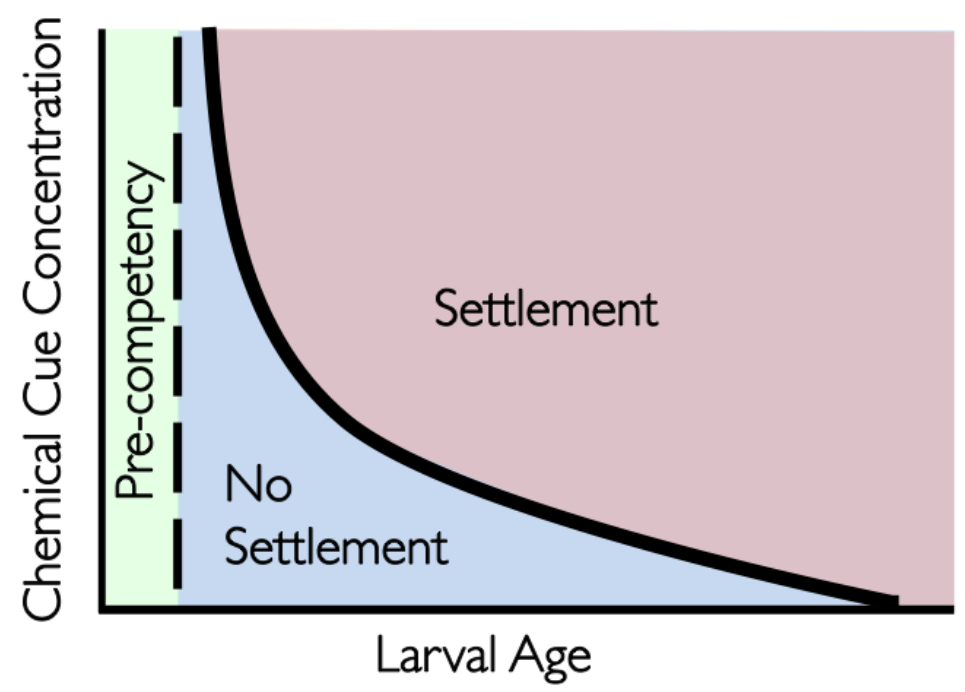

Figure 7-2: A schematic representation of the desperate larva hypothesis.

\subsubsection{Broader Impacts}

This work identifies relationships between environmental conditions, larval age, and resultant larval behavior. As such, it is of direct relevance to modellers attempting to parameterize larval behavior in large-scale biophysical dispersal models. Historically, larvae were treated as passive drifters in dispersal models, and only recently have modellers begun to grapple with behavioral effects [45]. My work can be used, for instance, to parameterize directional 
swimming in response to turbulence and chemical cues exuded from benthic settlement sites. It also accounts for diel changes in behavior, age effects, and morphology flow interactions. Moreover, my probabilistic model of larval diving is sufficiently general to incorporate the natural variability of the environmental signal (in this case, the local fluid acceleration). Addressing larval responses to rapidly varying natural environmental conditions is a real challenge in biophysical modelling, and my work offers one way to address this.

This conceptual framework for larval behavior discussed here relies heavily on our ability to distinguish larval behavior from the external forcing; for instance, larval swimming needs to be distinct from advection in ambient flow. The inability to distinguish these components of larval motion has previously limited studies of larval behavior in turbulence. In conjunction with my co-authors, I refined a method to simultaneously track larval motion and the turbulent flow field at high spatial and temporal resolution, using particle image velocimetry in conjunction with particle tracking. Similar methods were in use in related fields, quantifying weakly inertial particle motion in turbulence, as well as feeding and swimming currents in zooplankton. However, my work played a role in introducing PIV-based flow subtraction methods to the larval ecology community, and highlighting the breadth of scientific questions such a method could help investigate. 


\section{Bibliography}

[1] G Thorson. Light as an ecological factor in the dispersal and settlement of larvae of marine bottom invertebrates. Ophelia, 1:167-208, 1964.

[2] G Thorson. Reproductive and larval ecology of marine bottom invertebrates. Biological reviews, 25(1):1-45, 1950.

[3] MJ Kingsford, JM Leis, A Shanks, KC Lindeman, SG Morgan, and J Pineda. Sensory environments, larval abilities and local self-recruitment. Bulletin of Marine Science, 70(1):Suppl: 309-340, 2002.

[4] MG Hadfield and VJ Paul. Natural chemical cues for settlement and metamorphosis of marine invertebrate larvae. Marine Chemical Ecology, pages 431-461, 2001.

[5] RS Scheltema. On dispersal and planktonic larvae of benthic invertebrates: An eclectic overview and summary of problems. Bulletin of Marine Science, 39:290-322, 1986.

[6] VS Kennedy. Biology of larvae and spat. In VS Kennedy, RIE Newell, and AF Eble, editors, The Eastern Oyster (Crassostrea Virginica). Maryland Sea Grant, 1996.

[7] JA Pechenik. Biology of the Invertebrates, 7th ed. McGraw-Hill Education, 2014.

[8] RR Strathmann and D Grünbaum. Good eaters, poor swimmers: compromises in larval form. Integrative and Comparative Biology, 46(3):312-322, 2006.

[9] SM Gallager. Visual observations of particle manipulation during feeding in larvae of a bivalve mollusc. Bulletin of Marine Science, 43(3):344-365, 1988.

[10] HU Riisgård and PS Larsen. Particle capture mechanisms in suspension-feeding invertebrates. Marine Ecology Progress Series, 418:225-293, 2010.

[11] AL Shanks. Mechanisms of cross-shelf dispersal of larval invertebrates and fish. In L McEdward, editor, Ecology of marine invertebrate larvae, pages 323-367. CRC Press, Boca Raton, FL., 1995.

[12] A Metaxas. Behaviour in flow: Perspectives on the distribution and dispersion of meroplanktonic larvae in the water column. Canadian Journal of Fisheries and Aquatic Sciences, 58(1):86-98, 2001.

[13] LA Levin. Recent progress in understanding larval dispersal: new directions and digressions. Integrative and Comparative Biology, 46(3):282-297, 2006. 
[14] M Dekshenieks, E Hofmann, J Klinck, and E Powell. Modeling the vertical distribution of oyster larvae in response to environmental conditions. Marine Ecology Progress Series, 136(1):97-110, 1996.

[15] E North, Z Schlag, R Hood, M Li, L Zhong, T Gross, and VS Kennedy. Vertical swimming behavior influences the dispersal of simulated oyster larvae in a coupled particletracking and hydrodynamic model of Chesapeake Bay. Marine Ecology Progress Series, 359:99-115, 2008.

[16] C Kim, K Park, S Powers, W Graham, and K Bayha. Oyster larval transport in coastal Alabama: Dominance of physical transport over biological behavior in a shallow estuary. Journal of Geophysical Research, 115:C10019, 2010.

[17] KR Helrich and J Pineda. Accumulation of particles in propagating fronts. Limnology and Oceanography, 48(4):1509-1520, 2003.

[18] AL Shanks and L Brink. Upwelling, downwelling, and cross-shelf transport of bivalve larvae: Test of a hypothesis. Marine Ecology Progress Series, 302:1-12, 2005.

[19] A Scotti and J Pineda. Plankton accumulation and transport in propagating nonlinear internal fronts. Journal of Marine Research, 65(1):117-145, 2007.

[20] CB Woodson, MA McManus, JA Tyburczy, JA Barth, L Washburn, JE Caselle, MH Carr, DP Malone, PT Raimondi, BA Menge, and SR Palumbi. Coastal fronts set recruitment and connectivity pattern across multiple taxa. Limnology and Oceanography, 57(2):582-596, 2012.

[21] A McCulloch and AL Shanks. Topographically generated fronts, very nearshore oceanography and the distribution and settlement of mussel larvae and barnacle cyprids. Journal of Plankton Research, 25(11):1427-1439, 2003.

[22] AL Shanks, A McCulloch, and J Miller. Topographically generated fronts, very nearshore oceanography and the distribution of larval invertebrates and holoplankters. Journal of Plankton Research, 25(10):1251-1277, 2003.

[23] SR Rodriguez, FP Ojeda, and NC Inestrosa. Settlement of benthic marine invertebrates. Marine Ecology Progress Series, 97:193-207, 1993.

[24] A Abelson and M Denny. Settlement of marine organisms in flow. Annual Review of Ecology and Systematics, 28:317-339, 1997.

[25] HL Fuchs, LS Mullineaux, and AR Solow. Sinking behavior of gastropod larvae (Ilyanassa obsoleta) in turbulence. Limnology and Oceanography, 49:1937-1948, 2004.

[26] HL Fuchs and C DiBacco. Mussel larval responses to turbulence are unaltered by larval age or light conditions. Limnology and Oceanography: Fluids and Environment, $1: 120-134,2011$.

[27] HL Fuchs, EJ Hunter, EL Schmitt, and RA Guazzo. Active downward propulsion by oyster larvae in turbulence. The Journal of Experimental Biology, 216:1458-1469, 2013. 
[28] JD Wheeler, KR Helfrich, EJ Anderson, B McGann, P Staats, AE Wargula, K Wilt, and LS Mullineaux. Upward swimming of competent oyster larvae (Crassostrea virginica) persists in highly turbulent flow as detected by PIV flow subtraction. Marine Ecology Progress Series, 488:171-185, 2013.

[29] JD Wheeler, KR Helfrich, EJ Anderson, and LS Mullineaux. Isolating the hydrodynamic triggers of the dive response in eastern oyster larvae. Limnology and Oceanography, 60:1332-1343, 2015.

[30] A Lillis, DB Eggleston, and DR Bohnenstiehl. Oyster larvae settle in response to habitat-associated underwater sounds. PLoS ONE, 8(10):e79337, 2013.

[31] BL Bingham and CM Young. Larval phototaxis in barnacles and snails associated with bathyal sea urchins. Deep-Sea Research Part I, 40(1):1-12, 1993.

[32] RB Forward and TW Cronin. Tidal rhythms in activity and phototaxis by an estuarine crab larva. Biological Bulletin, 158:295-303, 1980.

[33] RSS Wu, PKS Lam, and BS Zhou. A phototaxis inhibition assay using barnacle larvae. Toxicology and Water Quality, 12(3):231-236, 1997.

[34] I Svane and CM Young. The ecology and behavior of ascidian larvae. Oceanography and Marine Biology, Annual Review, 27:45-90, 1989.

[35] E Vazquez and CM Young. Ontogenetic changes in phototaxis during larval life of the ascidian Polyandrocarpa zorritensis. Journal of Experimental Marine Biology and Ecology, 231:267-277, 1998.

[36] A Metaxas and V Burdett-Coutts. Response of invertebrate larvae to the presence of the ctenophore Bolinopsis infundibulum, a potential predator. Journal of Experimental Marine Biology and Ecology, 334(2):187-195, 2006.

[37] HU Dahms and PY Qian. Exposure of biofilms to meiofaunal copepods affects the larval settlement of Hydroides elegans (Polychaeta). Marine Ecology Progress Series, 297:203-214, 2005.

[38] MG Hadfield and JT Pennington. The nature of the metamorphic signal and its internal transduction in larvae of the nudibranch Phestilla sibogae. Bulletin of Marine Science, 46:445-464, 1990.

[39] G Botello and PJ Krug. Desperate larvae revisited: Age, energy and experience affect sensitivity to settlement cues in larvae of the gastropod Alderia sp. Marine Ecology Progress Series, 312:149-159, 2006.

[40] M Tamburri, R Zimmer-Faust, and M Tamplin. Natural sources and properties of chemical inducers mediating settlement of oyster larvae: A re-examination. The Biological Bulletin, 183:327-338, 1992.

[41] RJ Toonen and JR Pawlik. Settlement of the tube worm Hydroides dianthus (Polychaeta: Serpulidae): cues for gregarious settlement. Marine Biology, 126(4):725-733, 1996. 
[42] RK Cowen and S Sponaugle. Larval dispersal and marine population connectivity. Annual Review of Marine Science, 1:443-466, 2009.

[43] JA Pechenik. Larval experience and latent effectsâĂŤmetamorphosis is not a new beginning. Integrative and Comparative Biology, 46(3):323-333, 2006.

[44] J Pineda, JA Hare, and S Sponaungle. Larval transport and dispersal in the coastal ocean and consequences for population connectivity. Oceanography, 20:22-39, 2007.

[45] A Metaxas and M Saunders. Quantifying the âĂIJbio-âĂI components in biophysical models of larval transport in marine benthic invertebrates: advances and pitfalls. The Biological Bulletin, 216(3):257-272, 2009.

[46] TW Clay and D Grünbaum. Morphology-flow interactions lead to stage-selective vertical transport of larval sand dollars in shear flow. The Journal of Experimental Biology, 213(8):1281-1292, 2010.

[47] JA Sameoto, T Ross, and A Metaxas. The effect of flow on larval vertical distribution of the sea urchin, Strongylocentrotus droebachiensis. Journal of Experimental Marine Biology and Ecology, 383(2):156-163, 2010.

[48] MG Hadfield and MAR Koehl. Rapid behavioral responses of an invertebrate larva to dissolved settlement cue. The Biological Bulletin, 207(1):28-43, 2004.

[49] DS Wethey. Ranking of settlement cues by barnacle larvae: influence of surface contour. Bulletin of Marine Science, 39(2):393-400, 1986.

[50] RK Zimmer and CA Butman. Chemical signaling processes in the marine environment. The Biological Bulletin, 198(2):168-187, 2000.

[51] JE Eckman. Closing the larval loop: linking larval ecology to the population dynamics of marine benthic invertebrates. Journal of Experimental Marine Biology and Ecology, 200(1):207-237, 1996.

[52] A Roy, A Metaxas, and T Ross. Swimming patterns of larval Strongylocentrotus droebachiensis in turbulence in the laboratory. Marine Ecology Progress Series, 453:117$127,2012$.

[53] S Vogel. Life in moving fluids: the physical biology of flow. Princeton University Press, 1996.

[54] J Welch and R Forward. Flood tide transport of blue crab, Callinectes sapidus, postlarvae: behavioral responses to salinity and turbulence. Marine Biology, 139(5):911-918, 2001.

[55] C DiBacco, HL Fuchs, J Pineda, and KR Helfrich. Swimming behavior and velocities of barnacle cyprids in a downwelling flume. Marine Ecology Progress Series, 433:131$148,2011$.

[56] HL Fuchs, GP Gerbi, EJ Hunter, AJ Christman, and FJ Diez. Hydrodynamic sensing and behavior by oyster larvae in turbulence and waves. The Journal of Experimental biology, 218(9):1419-1432, 2015. 
[57] KYK Chan. Biomechanics of larval morphology affect swimming: Insights from the sand dollars Dendraster excentricus. Integrative and Comparative Biology, 54:458-469, 2012.

[58] JT Pennington and RR Strathmann. Consequences of the calcite skeletons of planktonic echinoderm larvae for orientation, swimming, and shape. The Biological Bulletin, 179(1):121-133, 1990.

[59] Y Mogami, J Ishii, and SA Baba. Theoretical and experimental dissection of gravitydependent mechanical orientation in gravitactic microorganisms. The Biological Bulletin, 201(1):26-33, 2001.

[60] AM Roberts. Geotaxis in motile micro-organisms. Journal of Experimental Biology, 53(3):687-699, 1970.

[61] JT Pennington and RB Emlet. Ontogenetic and diel vertical migration of a planktonic echinoid larva, Dendraster excentricus (eschscholtz): occurrence, causes, and probable consequences. Journal of Experimental Marine Biology and Ecology, 104(1):69-95, 1986.

[62] F De Lillo, M Cencini, WM Durham, M Barry, R Stocker, E Climent, and G Boffetta. Turbulent fluid acceleration generates clusters of gyrotactic microorganisms. Physical Review Letters, 112(4):044502, 2014.

[63] WM Durham, E Climent, M Barry, F De Lillo, G Boffetta, M Cencini, and R Stocker. Turbulence drives microscale patches of motile phytoplankton. Nature Communications, 4, 2013.

[64] WM Durham and R Stocker. Thin phytoplankton layers: characteristics, mechanisms, and consequences. Annual Review of Marine Science, 4:177-207, 2012.

[65] KYK Chan, E García, and S Dupont. Acidification reduced growth rate but not swimming speed of larval sea urchins. Scientific Reports, 5, 2015.

[66] KA McDonald. Earliest ciliary swimming effects vertical transport of planktonic embryos in turbulence and shear flow. The Journal of Experimental Biology, 215(1):141$151,2012$.

[67] HL Fuchs, AR Solow, and LS Mullineaux. Larval responses to turbulence and temperature in a tidal inlet: Habitat selection by dispersing gastropods? Journal of Marine Research, 68(1):153-188, 2010.

[68] JE Eckman. A model of passive settlement by planktonic larvae onto bottoms of differing roughness. Limnology and Oceanography, 35(4):887-901, 1990.

[69] CM Pearce, SM Gallager, JL Manuel, DA Manning, RK O’Dor, and E Bourget. Effect of thermoclines and turbulence on depth of larval settlement and spat recruitment of the giant scallop Placopecten magellanicus in $9.5 \mathrm{~m}$ deep laboratory mesocosms. Marine Ecology Progress Series, 1998.

[70] B Gaylord, J Hodin, and MC Ferner. Turbulent shear spurs settlement in larval sea urchins. Proceedings of the National Academy of Sciences, 110(17):6901-6906, 2013. 
[71] AJ Heyward and AP Negri. Turbulence, cleavage, and the naked embryo: a case for coral clones. Science, 335(6072):1064-1064, 2012.

[72] EB Harvey. The development of half and quarter eggs of Arbacia punctulata and of strongly centrifuged whole eggs. The Biological Bulletin, 62(2):155-167, 1932.

[73] RT Hinegardner. Growth and development of the laboratory cultured sea urchin. The Biological Bulletin, 137(3):465-475, 1969.

[74] D Nacci, J Serbst, TR Gleason, S Cayula, G Thursby, WR Munns Jr, and RK Johnston. Biological responses of the sea urchin, Arbacia punctulata, to lead contamination for an estuarine ecological risk assessment. Journal of Aquatic Ecosystem Stress and Recovery, 7(3):187-199, 2000.

[75] MF Strathmann. Reproduction and development of marine invertebrates of the northern Pacific coast: data and methods for the study of eggs, embryos, and larvae. University of Washington Press, 1987.

[76] TF Gross and ARM Nowell. Spectral scaling in a tidal boundary layer. Journal of Physical Oceanography, 15(5):496-508, 1985.

[77] J Schindelin, I Arganda-Carreras, E Frise, V Kaynig, M Longair, T Pietzsch, S Preibisch, C Rueden, S Saalfeld, B Schmid, et al. Fiji: an open-source platform for biological-image analysis. Nature Methods, 9(7):676-682, 2012.

[78] D Grünbaum and RR Strathmann. Form, performance and trade-offs in swimming and stability of armed larvae. Journal of Marine Research, 61(5):659-691, 2003.

[79] KYK Chan, D Grünbaum, and MJ O'Donnell. Effects of ocean-acidification-induced morphological changes on larval swimming and feeding. The Journal of Experimental Biology, 214(22):3857-3867, 2011.

[80] RB Emlet. Locomotion, drag, and the rigid skeleton of larval echinoderms. The Biological Bulletin, 164(3):433-445, 1983.

[81] TC Lacalli and THJ Gilmour. Ciliary reversal and locomotory control in the pluteus larva of Lytechinus pictus. Philosophical Transactions of the Royal Society of London B: Biological Sciences, 330(1258):391-396, 1990.

[82] T Kiørboe, H Jiang, RJ Gonçalves, LT Nielsen, and N Wadhwa. Flow disturbances generated by feeding and swimming zooplankton. Proceedings of the National Academy of Sciences, 111(32):11738-11743, 2014.

[83] LA Miller, DI Goldman, TL Hedrick, ED Tytell, ZJ Wang, J Yen, and S Alben. Using computational and mechanical models to study animal locomotion. Integrative and Comparative Biology, pages 553-575, 2012.

[84] Y Mogami, C Oobayashi, T Yamaguchi, Y Ogiso, and SA Baba. Negative geotaxis in sea urchin larvae: a possible role of mechanoreception in the late stages of development. Journal of Experimental Biology, 137(1):141-156, 1988.

[85] Y Wada, Y Mogami, and SA Baba. Modification of ciliary beating in sea urchin larvae induced by neurotransmitters: beat-plane rotation and control of frequency fluctuation. The Journal of Experimental Biology, 200(1):9-18, 1997. 
[86] T Gustafson and B Lundgren amd R Treufeldt. Serotonin and contractile activity in the echinopluteus: a study of the cellular basis of larval behaviour. Experimental Cell Research, 72(1):115-139, 1972.

[87] K Shiba, Y Mogami, and SA Baba. Ciliary movement of sea-urchin embryos. Nature Science Report, Ochanomizu University, 53:49-54, 2002.

[88] E Sodergren, GM Weinstock, EH Davidson, RA Cameron, RA Gibbs, RC Angerer, LM Angerer, MI Arnone, DR Burgess, RD Burke, et al. The genome of the sea urchin Strongylocentrotus purpuratus. Science, 314(5801):941-952, 2006.

[89] M Volnoukhin. Mechanosensory cells and swimming behaviour of embryos of the sea urchin Strongylocentrotus purpuratus. PhD thesis, Department of Molecular Biology and Biochemistry, Simon Fraser University, 2010.

[90] SM Gallager. Hydrodynamic disturbances produced by small zooplankton: case study for the veliger larva of a bivalve mollusc. Journal of Plankton Research, 15(11):12771296, 1993.

[91] CA Butman. Larval settlement of soft-sediment invertebrates: The spatial scales of pattern explained by active habitat selection and the emerging role of hydrodynamical processes. Oceanography and Marine Biology: An Annual Review, 25:113-165, 1987.

[92] CA Butman, JP Grassle, and CM Webb. Substrate choices made by marine larvae settling in still water and in a flume flow. Nature, 333:771-773, 1988.

[93] H Hidu and HH Haskin. Swimming speeds of oyster larvae Crassostrea virginica in different salinities and temperatures. Estuaries and Coasts, 1(4):252-255, 1978.

[94] HL Fuchs, MG Neubert, and LS Mullineaux. Effects of turbulence-mediated larval behavior on larval supply and settlement in tidal currents. Limnology and Oceanography, pages 1156-1165, 2007.

[95] MN Tamburri, CM Finelli, DS Wethey, and RK Zimmer-Faust. Chemical induction of larval settlement behavior in flow. The Biological Bulletin, 191(3):367-373, 1996.

[96] CM Finelli and DS Wethey. Behavior of oyster (Crassostrea virginica) larvae in flume boundary layer flows. Marine Biology, 143(4):703-711, 2003.

[97] ER Whitman and MA Reidenbach. Benthic flow environments affect recruitment of Crassostrea virginica larvae to an intertidal oyster reef. Marine Ecology Progress Series, 463:177-191, 2012.

[98] MAR Koehl and MA Reidenbach. Swimming by microscopic organisms in ambient water flow. Experiments in Fluids, 43(5):755-768, 2007.

[99] MAR Koehl and MG Hadfield. Hydrodynamics of larval settlement from a larvaâẮ́s point of view. Integrative and Comparative Biology, 50(4):539-551, 2010.

[100] R George, RE Flick, and RT Guza. Observations of turbulence in the surf zone. Journal of Geophysical Research, 99:801-810, 1994. 
[101] VS Kennedy. Expected seasonal presence of Crassostrea virginica (Gmelin) larval populations, emphasizing Chesapeake Bay. American Malacological Bulletin, Special Edition, (3):25-29, 1986.

[102] PS Galtsoff. The American oyster Crassostrea virginica Gmelin. US Fish and Wildlife Service Fishery Bulletin, 64:1-480, 1964.

[103] RJ Thompson, RIE Newell, VS Kennedy, and R MAnn. Reproductive processes and early development. In VS Kennedy, RIE Newell, and AF Eble, editors, The Eastern Oyster (Crassostrea Virginica). Maryland Sea Grant, 1996.

[104] P Doron, L Bertuccioli, J Katz, and TR Osborn. Turbulence characteristics and dissipation estimates in the coastal ocean bottom boundary layer from PIV data. Journal of Physical Oceanography, 31(8):2108-2134, 2001.

[105] M Raffel, CE Willert, and J Kompenhans. Particle Image Velocimetry: A Practical Guide. Springer-Verlag, 1998.

[106] GV Glass, PD Peckham, and JR Sanders. Consequences of failure to meet assumptions underlying the fixed effects analyses of variance and covariance. Review of Educational Research, 42(3):237-288, 1972.

[107] F Yates. Contingency tables involving small numbers and the $\chi^{2}$ test. Supplement to the Journal of the Royal Statistical Society, 1(2):217-235, 1934.

[108] J Davila and JCR Hunt. Settling of small particles near vortices and in turbulence. Journal of Fluid Mechanics, 440(117-145):384, 2001.

[109] S Balachandar. A scaling analysis for point-particle approaches to turbulent multiphase flows. International Journal of Multiphase Flow, 35(9):801-810, 2009.

[110] S Balachandar and JK Eaton. Turbulent dispersed multiphase flow. Annual Review of Fluid Mechanics, 42:111-133, 2010.

[111] EW Knight-Jones. Laboratory experiments on gregariousness during setting in Balanus balanoides and other barnacles. Journal of Experimental Biology, 30(4):584-598, 1953.

[112] T Kiørboe, E Saiz, and A Visser. Hydrodynamic signal perception in the copepod Acartia tonsa. Marine Ecology Progress Series, 179:97-111, 1999.

[113] HH Jakobsen. Escape response of planktonic protists to fluid mechanical signals. Marine Ecology Progress Series, 214:67-78, 2001.

[114] R Holzman and PC Wainwright. How to surprise a copepod: strike kinematics reduce hydrodynamic disturbance and increase stealth of suction-feeding fish. Limnology and Oceanography, 54(6):2201-2212, 2009.

[115] PR Jonsson, C Andre, and M Lindegarth. Swimming behavior of marine bivalve larvae in a flume boundary-layer flow: evidence for near-bottom confinement. Marine Ecology Progress Series, 79(1-2):67-76, 1991.

[116] TJ Pedley and JO Kessler. Hydrodynamic phenomena in suspensions of swimming microorganisms. Annual Review of Fluid Mechanics, 24(1):313-358, 1992. 
[117] ARM Nowell and PA Jumars. Flow environments of aquatic benthos. Annual Review of Ecology and Systematics, pages 303-328, 1984.

[118] MAR Koehl. Mini review: Hydrodynamics of larval settlement into fouling communities. Biofouling, 23(5):357-368, 2007.

[119] T Kiørboe and AW Visser. Predator and prey perception in copepods due to hydromechanical signals. Marine Ecology Progress Series, 179:81-95, 1999.

[120] FS Chia, R Koss, and LR Bickell. Fine structural study of the statocysts in the veliger larva of the nudibranch, Rostanga pulchra. Cell and Tissue Research, 214(1):67-80, 1981.

[121] MR Maxey and JJ Riley. Equation of motion for a small rigid sphere in a nonuniform flow. Physics of Fluids, 26(4):883-889, 1983.

[122] MAR Koehl, JA Strother, MA Reidenbach, JR Koseff, and MG Hadfield. Individualbased model of larval transport to coral reefs in turbulent, wave-driven flow: behavioral responses to dissolved settlement inducer. Marine Ecology Progress Series, 335:1-18, 2007.

[123] K Troost, R Veldhuizen, EJ Stamhuis, and WJ Wolff. Can bivalve veligers escape feeding currents of adult bivalves? Journal of Experimental Marine Biology and Ecology, 358(2):185-196, 2008.

[124] RE Pepper, JS Jaffe, E Variano, and MAR Koehl. Zooplankton in flowing water near benthic communities encounter rapidly fluctuating velocity gradients and accelerations. Marine Biology, 162(10):1939-1954, 2015.

[125] RJ Waggett and EJ Buskey. Calanoid copepod escape behavior in response to a visual predator. Marine Biology, 150(4):599-607, 2007.

[126] CH Lee, HU Dahms, SH Cheng, S Souissi, FG Schmitt, R Kumar, and JS Hwang. Predation of Pseudodiaptomus annandalei (copepoda: Calanoida) by the grouper fish fry Epinephelus coioides under different hydrodynamic conditions. Journal of Experimental Marine Biology and Ecology, 393(1):17-22, 2010.

[127] AK Awasthi and $\mathrm{CH}$ Wu amd JS Hwang. Diving as an anti-predator behavior in mosquito pupae. Zoological Studies, 51(8):1225-1234, 2012.

[128] RIE Newell. Ecological changes in Chesapeake Bay: Are they the result of overharvesting the American oyster, Crassostrea virginica. Understanding the Estuary: Advances in Chesapeake Bay Research, 129:536-546, 1988.

[129] DL Breitburg, LD Coen, MW Luckenbach, R Mann Roger, M Posey, and JA Wesson. Oyster reef restoration: convergence of harvest and conservation strategies. Journal of Shellfish Research, 19(1):371-377, 2000.

[130] KA Nelson, LA Leonard, MH Posey, TD Alphin, and MA Mallin. Using transplanted oyster (Crassostrea virginica) beds to improve water quality in small tidal creeks: a pilot study. Journal of Experimental Marine Biology and Ecology, 298(2):347-368, 2004. 
[131] BJ Rothschild, JS Ault, P Goulletquer, and M Heral. Decline of the Chesapeake Bay oyster population: a century of habitat destruction and overfishing. Marine Ecology Progress Series, 111(1-2):29-39, 1994.

[132] WM Kemp, WR Boynton, JE Adolf, DF Boesch, WC Boicourt, G Brush, JC Cornwell, TR Fisher, PM Glibert, JD Hagy, et al. Eutrophication of Chesapeake Bay: historical trends and ecological interactions. Marine Ecology Progress Series, 303(21):1-29, 2005.

[133] CK Kim, K Park, and SP Powers. Establishing restoration strategy of eastern oyster via a coupled biophysical transport model. Restoration Ecology, 21(3):353-362, 2013.

[134] IK Bartol, R Mann, and M Luckenbach. Growth and mortality of oysters (Crassostrea virginica) on constructed intertidal reefs: effects of tidal height and substrate level. Journal of Experimental Marine Biology and Ecology, 237(2):157-184, 1999.

[135] JA Nestlerode, MW Luckenbach, and FX O'Beirn. Settlement and survival of the oyster Crassostrea virginica on created oyster reef habitats in Chesapeake Bay. Restoration Ecology, 15(2):273-283, 2007.

[136] Eastern Oyster Biological Team. Status review of the eastern oyster (Crassostrea virginica). Report to the National Marine Fisheries Service, Northeast Regional Office, 2007.

[137] DW Fredriksson, CN Steppe, L Wallendorf, S Sweeney, and D Kriebel. Biological and hydrodynamic design considerations for vertically oriented oyster grow out structures. Aquacultural Engineering, 42(2):57-69, 2010.

[138] CM Young, M Craig, and FU Chia. Ontogeny of phototaxis during larval development of the sedentary polychaete, Serpula vermicularis (1.). The Biological Bulletin, $162(3): 457-468,1982$.

[139] AW Visser. Motility of zooplankton: fitness, foraging and predation. Journal of Plankton research, 29(5):447-461, 2007.

[140] Y Iwasa. Vertical migration of zooplankton: a game between predator and prey. American Naturalist, pages 171-180, 1982.

[141] G Jékely, J Colombelli, H Hausen, K Guy, E Stelzer, F Nédélec, and D Arendt. Mechanism of phototaxis in marine zooplankton. Nature, 456(7220):395-399, 2008.

[142] G Jékely. Evolution of phototaxis. Philosophical Transactions of the Royal Society B: Biological Sciences, 364(1531):2795-2808, 2009.

[143] KYK Chan and D Grünbaum. Temperature and diet modified swimming behaviors of larval sand dollar. Marine Ecology Progress Series, 415:49-59, 2010.

[144] Y Yamada, A Okamura, N Mikawa, T Utoh, Noriyuki N Horie, S Tanaka, MJ Miller, and $\mathrm{K}$ Tsukamoto. Ontogenetic changes in phototactic behavior during metamorphosis of artificially reared Japanese eel Anguilla japonica larvae. Marine Ecology Progress Series, 379:241-251, 2009.

[145] DA McCarthy, RB Forward, and CM Young. Ontogeny of phototaxis and geotaxis during larval development of the sabellariid polychaete Phragmatopoma lapidosa. Marine Ecology Progress Series, 241:215-220, 2002. 
[146] RB Forward and JD Costlow. The ontogeny of phototaxis by larvae of the crab Rhithropanopeus harrisii. Marine Biology, 26(1):27-33, 1974.

[147] SE Miller and MG Hadfield. Ontogeny of phototaxis and metamorphic competence in larvae of the nudibranch Phestilla sibogae Bergh (Gastropoda: Opisthobranchia). Journal of Experimental Marine Biology and Ecology, 97(1):95-112, 1986.

[148] PJ Barile, AW Stoner, and CM Young. Phototaxis and vertical migration of the queen conch (|emphStrombus gigas linne) veliger larvae. Journal of Experimental Marine Biology and Ecology, 183(2):147-162, 1994.

[149] G Champalbert, C Macquart-Moulin, G Patriti, and D Chiki. Ontogenic variations in the phototaxis of larval and juvenile sole Solea solea L. Journal of Experimental Marine Biology and Ecology, 149(2):207-225, 1991.

[150] RD Burke. Pheromones and the gregarious settlement of marine invertebrate larvae. Bulletin of Marine Science, 39(2):323-331, 1986.

[151] JR Pawlik. Chemical ecology of the settlement of benthic marine invertebrates. Oceanography and Marine Biology Annual Review, 30:273-335, 1992.

[152] DJ Crisp. Chemical factors inducing settlement in Crassostrea virginica (gmelin). The Journal of Animal Ecology, pages 329-335, 1967.

[153] RK Zimmer-Faust and MN Tamburri. Chemical identity and ecological implications of a waterborne, larval settlement cue. Limnology and Oceanography, 39(5):1075-1087, 1994.

[154] WK Fitt, SL Coon, M Walch, RM Weiner, RR Colwell, and DB Bonar. Settlement behavior and metamorphosis of oyster larvae (Crassostrea gigas) in response to bacterial supernatants. Marine Biology, 106(3):389-394, 1990.

[155] WK Fitt and SL Coon. Evidence for ammonia as a natural cue for recruitment of oyster larvae to oyster beds in a Georgia salt marsh. The Biological Bulletin, 182(3):401-408, 1992.

[156] DM Schulte, RP Burke, and RN Lipcius. Unprecedented restoration of a native oyster metapopulation. Science, 325(5944):1124-1128, 2009.

[157] AH Campbell, DW Meritt, RB Franklin, EL Boone, CT Nicely, and BL Brown. Effects of age and composition of field-produced biofilms on oyster larval setting. Biofouling, 27(3):255-265, 2011.

[158] EW Knight-Jones. Gregariousness and some other aspects of the setting behaviour ofSipirorbis. Journal of the Marine Biological Association of the United Kingdom, 30(2):201-222, 1951.

[159] DP Wilson. The settlement of Ophelia Bicornis Savigny larvae. Journal of the Marine Biological Association of the United Kingdom, 32(1):209-233, 1953.

[160] CD Bishop, MJ Huggett, A Heyland, J Hodin, and BP Brandhorst. Interspecific variation in metamorphic competence in marine invertebrates: the significance for comparative investigations into the timing of metamorphosis. Integrative and Comparative Biology, 46(6):662-682, 2006. 
[161] PE Gribben, DJ Marshall, and PD Steinberg. Less inhibited with age? larval age modifies responses to natural settlement inhibitors. Biofouling, 22(02):101-106, 2006.

[162] G Gibson. Why be choosy? Temporal changes in larval sensitivity to several naturallyoccurring metamorphic inducers in the opisthobranch Haminaea callidegenita. Journal of Experimental Marine Biology and Ecology, 194(1):9-24, 1995.

[163] DJ Marshall and MJ Keough. Variation in the dispersal potential of non-feeding invertebrate larvae: the desperate larva hypothesis and larval size. Marine Ecology Progress Series, 255:145-153, 2003.

[164] C Elkin and DJ Marshall. Desperate larvae: influence of deferred costs and habitat requirements on habitat selection. Marine Ecology Progress Series, 335:143-153, 2007.

[165] RL Swanson, DJ, and PD Steinberg. Larval desperation and histamine: how simple responses can lead to complex changes in larval behaviour. Journal of Experimental Biology, 210(18):3228-3235, 2007.

[166] Z Su, L Huang, Y Yan, and H Li. The effect of different substrates on pearl oyster Pinctada martensii (Dunker) larvae settlement. Aquaculture, 271(1):377-383, 2007.

[167] SC Burgess, SP Hart, and DJ Marshall. Pre-settlement behavior in larval bryozoans: the roles of larval age and size. The Biological Bulletin, 216(3):344-354, 2009.

[168] JT Pennington, SS Rumrill, and FS Chia. Stage-specific predation upon embryos and larvae of the Pacific sand dollar, Dendraster excentricus, by 11 species of common zooplanktonic predators. Bulletin of Marine Science, 39(2):234-240, 1986.

[169] SS Rumrill. Natural mortality of marine invertebrate larvae. Ophelia, 32(1-2):163-198, 1990 .

[170] R Beaz-Hidalgo, S Balboa, JL Romalde, and MJ Figueras. Diversity and pathogenecity of Vibrio species in cultured bivalve molluscs. Environmental Microbiology Reports, 2(1):34-43, 2010.

[171] GA Jackson and RR Strathmann. Larval mortality from offshore mixing as a link between precompetent and competent periods of development. American Naturalist, pages 16-26, 1981.

[172] P Richerson, R Armstrong, and CR Goldman. Contemporaneous disequilibrium, a new hypothesis to explain the "paradox of the plankton". Proceedings of the National Academy of Sciences, 67(4):1710-1714, 1970.

[173] R Lasker. Field criteria for survival of anchovy larvae-relation between inshore chlorophyll maximum layers and successful 1st feeding. Fishery Bulletin, 73(3):453-462, 1975.

[174] RJ Toonen and JR Pawlik. Settlement of the gregarious tube worm Hydroides dianthus (Polychaeta: Serpulidae). ii. testing the desperate larva hypothesis. Marine Ecology Progress Series, 224:115-131, 2001.

[175] EB May. A survey of the oyster and oyster shell resources of Alabama. Alabama Marine Resource Bulletin, 4, 1971. 